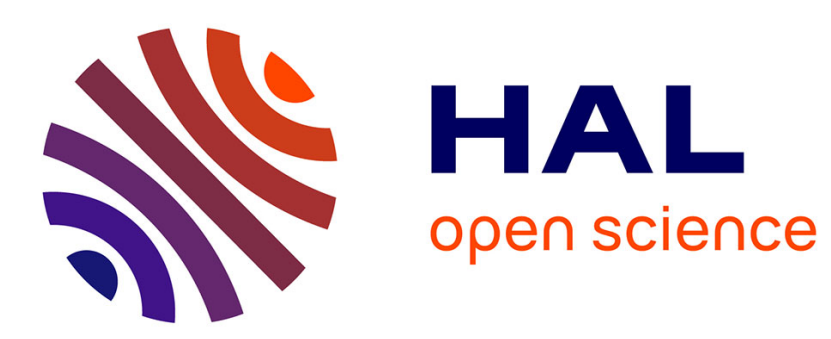

\title{
Kantorovich-Rubinstein Quasi-Metrics I: Spaces of Measures and of Continuous Valuations
}

\author{
Jean Goubault-Larrecq
}

\section{To cite this version:}

Jean Goubault-Larrecq. Kantorovich-Rubinstein Quasi-Metrics I: Spaces of Measures and of Continuous Valuations. Topology and its Applications, 2021, 10.1016/j.topol.2021.107673 . hal-03186371

\section{HAL Id: hal-03186371 \\ https://hal.science/hal-03186371}

Submitted on 9 Jun 2021

HAL is a multi-disciplinary open access archive for the deposit and dissemination of scientific research documents, whether they are published or not. The documents may come from teaching and research institutions in France or abroad, or from public or private research centers.
L'archive ouverte pluridisciplinaire HAL, est destinée au dépôt et à la diffusion de documents scientifiques de niveau recherche, publiés ou non, émanant des établissements d'enseignement et de recherche français ou étrangers, des laboratoires publics ou privés. 


\title{
Kantorovich-Rubinstein Quasi-Metrics I: Spaces of Measures and of Continuous Valuations
}

\author{
Jean Goubault-Larrecq \\ Université Paris-Saclay, CNRS, ENS Paris-Saclay, Laboratoire méthodes formelles, \\ 91190, Gif-sur-Yvette, France. \\ goubault@lsv.fr
}

\begin{abstract}
We show that the space of subprobability measures, equivalently of subprobability continuous valuations, on an algebraic (resp., continuous) complete quasi-metric space is again algebraic (resp., continuous) and complete, when equipped with the Kantorovich-Rubinstein quasi-metrics $d_{\mathrm{KR}}$ (unbounded) or $d_{\mathrm{KR}}^{a}$ (bounded), themselves asymmetric forms of the well-known KantorovichRubinstein metric. We also show that the $d_{\mathrm{KR}}$-Scott and the $d_{\mathrm{KR}}^{a}-\mathrm{Scott}$ topologies then coincide with the weak topology. We obtain similar results for spaces of probability measures, equivalently of probability continuous valuations, with the $d_{\mathrm{KR}}^{a}$ quasi-metrics, or with the $d_{\mathrm{KR}}$ quasi-metric under an additional rootedness assumption.
\end{abstract}

Keywords: Quasi-metric, continuous valuation, Kantorovich-Rubinstein quasi-metric, weak topology 2020 MSC: 54H30, 28A33, 46E27

\section{Introduction}

Kantorovich-Rubinstein metrics are $L^{1}$-like metrics on spaces of probability measures, and have a number of pleasing properties. Notably, they are complete separable if the underlying metric space of points is complete separable, and in that case they metrize the weak topology.

We adapt this to the case of quasi-metrics, which are asymmetric variants on the notion of metrics. Specifically, we are interested in KantorovichRubinstein-like quasi-metrics. In the first of two kinds, we will define the distance from the measure $\mu$ on the quasi-metric space $X, d$ to $\nu$ as a supre- 
mum over all 1-Lipschitz continuous maps $f: X \rightarrow \mathbb{R}_{+}$of $\max \left(\int_{x \in X} f(x) d \mu-\right.$ $\left.\int_{x \in X} f(x) d \nu, 0\right)$. The usual definition in metric spaces would use absolute value instead of $\max (-, 0)$; the two definitions are equivalent when $d$ is a metric, but the former definition is more suited to the asymmetric case of quasi-metric spaces.

As we will see, the results about completeness and the weak topology mentioned earlier generalize neatly to the quasi-metric case. However, this comes at a price. First, there will be a number of subtle points about quasimetric spaces that one needs to be aware of, and which do not crop up with metric spaces (see Section 2.2). Second, the reader should be warned that the technical developments are rather different than in the classical, metric case, and more demanding.

We also work with continuous valuations instead of measures: those are close cousins, which tend to interact more naturally with topology. In the case that will interest us most, namely on continuous complete quasi-metric spaces, bounded continuous valuations and $\tau$-smooth measures can safely be equated; we will give the precise statement in Section 2.3. Hence, and although the title of this paper mentions measures, we will work with continuous valuations, almost exclusively, throughout; almost, because we will need to cross back the bridge leading to measures in a crucial step (see Section 4.5).

This work is part one of a study of quasi-metrics on spaces of so-called previsions [14], of which this is the linear case. The non-linear cases, which model not just probabilistic choice, but also various forms of non-deterministic choice and mixed, probabilistic and non-deterministic choice, will be explored in subsequent parts ${ }^{1}$.

Outline. We recapitulate some preliminary definitions and notions in Section 2. This is probably most needed for the theory of quasi-metric spaces and their $d$-Scott topology, which is relatively new and not entirely well-known. For example, a Lipschitz map may fail to be continuous with respect to these topologies. As another illustration, among the quasi-metric spaces, we have the complete spaces (called Yoneda-complete in [3]), the strictly smaller class of continuous complete spaces, the even smaller class of algebraic complete spaces, and the even smaller class of Smyth-complete spaces-and all those

\footnotetext{
${ }^{1}$ An unpublished version of that work is available on arXiv [17]. The present paper roughly covers Sections 10, 6.3, and 11-except for 11.9 and 11.10 - in that order.
} 
notions would coincide on metric spaces.

We introduce the unbounded and bounded versions, $d_{\mathrm{KR}}$ and $d_{\mathrm{KR}}^{a}$, of our so-called Kantorovich-Rubinstein quasi-metrics on continuous valuations in Section 3. In Section 4, we show that the spaces of (continuous, resp. subprobability, resp. probability) valuations on a Lipschitz regular space $X, d$ are complete, when equipped with the $d_{\mathrm{KR}}$ or the $d_{\mathrm{KR}}^{a}$ quasi-metric. Using formal balls and measure extension theorems, we obtain similar completeness theorems for spaces of (sub)probability valuations if $X, d$ is continuous complete instead. Note that this does not say (yet) that our spaces of valuations are continuous complete in that case.

Before we deal with continuity, we look at the more constrained case of algebraic complete quasi-metric spaces. In Section 5, we show that the space of subprobability valuations on an algebraic complete quasi-metric space is again algebraic complete, both with $d_{\mathrm{KR}}$ and with $d_{\mathrm{KR}}^{a}$. The case of probability valuations is more intricate, and we show that the space of probability valuations on an algebraic complete space $X, d$ is again algebraic complete, either for the $d_{\mathrm{KR}}^{a}$ quasi-metric with no additional assumption, or for the $d_{\mathrm{KR}}$ quasi-metric under the extra assumption that $X, d$ has a root, a situation that encompasses the case where the quasi-metric $d$ is bounded.

An additional case where we obtain algebraic completeness is when $X, d$ is complete metric. In that case, as we show in Section 7, our $d_{\mathrm{KR}}$ and $d_{\mathrm{KR}}^{a}$ quasi-metrics are metrics already. In that, they coincide with the usual Kantorovich-Rubinstein metrics, and algebraicity is for free -all complete metric spaces are algebraic complete.

In Section 8, we show that the space of subprobability valuations on a continuous complete quasi-metric space $X, d$ is again continuous complete, both in $d_{\mathrm{KR}}$ and in $d_{\mathrm{KR}}^{a}$, and that the same happens to the space of probability valuations under the usual assumptions: using the $d_{\mathrm{KR}}^{a}$ quasi-metric, or the $d_{\mathrm{KR}}$ quasi-metric in case $X, d$ is rooted (or the $d_{\mathrm{KR}}$ metric in case $d$ is a metric).

In Section 9, we show that the $d_{\mathrm{KR}}^{a}$-Scott topologies on spaces of (sub)probability valuations on algebraic complete spaces, and more generally on continuous complete spaces, coincide with the weak topology. The $d_{\mathrm{KR}}-\mathrm{Scott}$ topology differs, even on complete metric spaces, and we recall a counterexample due to Kravchenko [26]. We conclude by listing a few open problems in Section 10.

We sum up our main results in the following tables; "cc" abbreviates "continuous complete", and "ac" abbreviates "algebraic complete". 


\begin{tabular}{|l|c|c|c|}
\hline$Y=\mathbf{V}_{\leq 1} X$ & $X$ ac & $X$ cc & $d$-Scott=weak? \\
\hline \hline$d_{\mathrm{KR}}$ & $Y$ ac & $Y$ cc & no \\
\hline$d_{\mathrm{KR}}^{a}$ & $Y$ ac & $Y$ cc & yes \\
\hline
\end{tabular}

\begin{tabular}{|l|c|c|c|}
\hline$Y=\mathbf{V}_{1} X$ & $X$ ac & $X$ cc & $d$-Scott=weak? \\
\hline \hline$d_{\mathrm{KR}}$ & $\begin{array}{c}Y \text { complete, } \\
\text { not known to be ac* }\end{array}$ & $\begin{array}{c}Y \text { complete, } \\
\text { not known to be cc* }\end{array}$ & no \\
\hline$d_{\mathrm{KR}}^{a}$ & $Y$ ac & $Y$ cc & yes \\
\hline \multicolumn{2}{|c|}{$\left({ }^{*}\right.$ unless $X, d$ has a root, e.g. $d$ is bounded, or $X, d$ is metric.) }
\end{tabular}

Related work. Prokhorov was the first who showed that the space of probability measures on a Polish space is itself Polish [28]. In other words, given a complete separable metric space $X, d$, there is a metric on the space of probability measures on $X$ that makes it complete and separable, and whose open ball topology is the weak topology [28, Lemma 1.4]. Instead of the Prokhorov (also called Lévy-Prokhorov) metric, one can use the Vaseršteŭn metric $W^{p}$, defined when $p \geq 1$ by $W^{p}(\mu, \nu)=\left(\inf _{\varpi} \int_{(x, y) \in X^{2}} d^{p}(x, y) d \varpi\right)^{1 / p}$, where $\varpi$ ranges over the probability measures on $X^{2}$ whose first and second marginals are $\mu$ and $\nu$ respectively. If $X, d$ is complete separable, then $W^{p}$ is complete separable. A nice proof of this completeness result is due to Bolley [2]. The topology of $W^{p}$ is also the weak topology - if $d$ is bounded.

The Kantorovich-Rubinstein duality theorem states that $W^{1}(\mu, \nu)$ is equal to $d_{\mathrm{KR}}(\mu, \nu)=\sup _{h}\left|\int h d \mu-\int h d \nu\right|$, where $h$ ranges over the 1-Lipschitz maps from $X$ to $\overline{\mathbb{R}}_{+}$, provided that $X$ is Polish. This was originally proved for compact Hausdorff spaces [23, 24], and later extended to Polish spaces $[8,5,12]$. The latter sup formula is our model for our so-called KantorovichRubinstein quasi-metrics, to be introduced in Section 3. As a consequence, $d_{\mathrm{KR}}$ is also a complete separable metric on the space of probabilities on a complete separable metric space.

The only Kantorovich-Rubinstein duality theorem that we know on quasimetric spaces is Theorem 5 of $[15]^{2}$, and it only applies to so-called symcompact spaces $X, d$, namely to quasi-metric spaces such that $X$ is compact in the symmetrized metric $d^{\text {sym }}(x, y)=\max (d(x, y), d(y, x))$. We have no such duality theorem on the more general classes of quasi-metric spaces which we

\footnotetext{
${ }^{2}$ Another attempt is given in Section 11.10 of [17], but this is a very weak result.
} 
will consider here, and therefore we will provide direct proofs of completeness. In the metric case, there is at least one direct proof of completeness of $d_{\mathrm{KR}}$, namely without any detour through another metric, due to Kravchenko [26], but our proof is necessarily very different.

Finally, we should mention Ph. Sünderhauf's results [29]. He introduces a so-called sup quasi-metric, which is somewhat similar to the usual total variation metric. He then shows that the spaces of continuous valuations (resp., probability, subprobability continuous valuations) on an algebraic dcpo $X$ are algebraic complete quasi-metric spaces under the sup quasi-metric [29, Theorem 3.4]. Although the sup quasi-metric is very different from the $d_{\mathrm{KR}}$ and $d_{\mathrm{KR}}^{a}$ metrics on metric spaces, we will see that it is exactly our quasimetric $d_{\mathrm{KR}}^{1}$ on posets: see Remark 3.6.

\section{Preliminaries}

\subsection{General topology}

We refer the reader to [16] for basic notions and theorems of topology, domain theory, and in the theory of quasi-metric spaces. The book [13] is the standard reference on domain theory, and I will assume known the notions of directed complete posets (dcpo), Scott-continuous functions, the way-below relation $\ll$, continuous posets and dcpos, and so on. Additionally, this paper will heavily rely on [20] for additional results on quasi-metric spaces, on [18] for formal ball monads, and on [19] for results on spaces of Lipschitz continuous maps; we will recall the required results as needed.

We write $\uparrow x$ for the set of points $y$ such that $x \ll y$. The Scott topology on a poset consists of the Scott-open subsets, namely the upwards-closed subsets $U$ such that every directed family that has a supremum in $U$ must intersect $U$. A Scott-continuous map between posets is one that is monotonic and preserves existing directed suprema, and this is equivalent to requiring that it is continuous for the underlying Scott topologies.

The focus of the present paper is on quasi-metric spaces. Chapters 6 and 7 of [16] are a recommended read on that subject. The paper [20] gives additional information on quasi-metric spaces, which we will also rely on.

As far as topology is concerned, compactness does not imply separation. In other words, we call a subset $K$ of a topological space compact if and only if every open cover of $K$ contains a finite subcover. This property is sometimes called quasicompactness. 
We shall always write $\leq$ for the specialization preordering of a topological space: $x \leq y$ if and only if every open neighborhood of $x$ is also an open neighborhood of $y$, if and only if $x$ is in the closure of $y$. As a result, the closure of a single point $y$ is also its downward closure $\downarrow y$. In general, we write $\downarrow A$ for the downward closure of any set $A, \uparrow A$ for its upward closure, and $\uparrow x=\uparrow\{x\}$. A saturated subset of a topological space is a set that is the intersection of all its open neighborhoods; equivalently, it is an upwardsclosed subset in its specialization preordering.

In a topological space, $\uparrow A$ is also equal to the saturation of $A$, namely the intersection of all the open neighborhoods of $A$. If $K$ is compact, then so is its saturation $\uparrow K$, and we shall usually use the letter $Q$ for compact saturated subsets.

\subsection{Quasi-metric spaces}

Let $\overline{\mathbb{R}}_{+}$be the set of extended non-negative reals. A quasi-metric on a set $X$ is a map $d: X \times X \rightarrow \overline{\mathbb{R}}_{+}$satisfying: $d(x, x)=0 ; d(x, z) \leq d(x, y)+d(y, z)$ (triangular inequality); $d(x, y)=d(y, x)=0$ implies $x=y$. The pair $X, d$ is then called a quasi-metric space. Given $X, d$, there is an ordering $\leq^{d}$ on $X$ given by $x \leq^{d} y$ if and only if $d(x, y)=0$.

A trivial example is $\overline{\mathbb{R}}_{+}$itself, with the quasi-metric $\mathrm{d}_{\mathbb{R}}$ defined by $\mathrm{d}_{\mathbb{R}}(x, y)=$ 0 if $x \leq y, \mathrm{~d}_{\mathbb{R}}(+\infty, y)=+\infty$ if $y \neq+\infty, \mathrm{d}_{\mathbb{R}}(x, y)=x-y$ if $x>y$ and $x \neq+\infty$. Then $\leq \mathrm{d}_{\mathbb{R}}$ is the ordinary ordering $\leq$.

Every metric is a quasi-metric. At the other end of the spectrum, every partial ordering $\leq$ on a set $X$ defines a quasi-metric $d_{\leq}$by $d_{\leq}(x, y)=0$ if and only if $x \leq y,+\infty$ otherwise. Conversely, every quasi-metric $d$ that takes its values in $\{0,+\infty\}$ is equal to $d_{\leq}$for some unique ordering $\leq$(namely $\leq^{d}$ ).

The space of formal balls $\mathbf{B}(X, d)$ of a quasi-metric space $X, d$ is probably the single most important artifact that has to be considered in the study of quasi-metric spaces $[31,10]$. This has a very simple definition: a formal ball is syntax for an actual ball, namely a pair $(x, r)$ where $x \in X$ (the center) and $r \in \mathbb{R}_{+}$(the radius). $\mathbf{B}(X, d)$ comes with an ordering $\leq^{d^{+}}$defined by $(x, r) \leq d^{+}(y, s)$ if and only if $d(x, y) \leq r-s$.

Given a quasi-metric space $X, d$, the open ball $B_{x,<r}^{d}$ with center $x \in X$ and radius $r \in \mathbb{R}_{+}$is $\{y \in X \mid d(x, y)<r\}$. The open ball topology is the coarsest containing all open balls, and is the standard topology on metric spaces.

In the realm of quasi-metric spaces, the $d$-Scott topology is the topology we shall always consider, unless specified otherwise. This is defined as follows. 
We equip $\mathbf{B}(X, d)$ with the Scott topology of $\leq^{d^{+}}$. There is an injective map $\eta_{X}: x \mapsto(x, 0)$ from $X$ to $\mathbf{B}(X, d)$, and the $d$-Scott topology is the coarsest that makes it continuous. This allows us to see $X$ as a topological subspace of $\mathbf{B}(X, d)$.

The specialization ordering of $X, d$, whether with the open ball topology or with the $d$-Scott topology, is just $\leq^{d}$. This does not cause any conflict with the notation $\leq{ }^{d^{+}}$for the ordering on $\mathbf{B}(X, d)$, since $\leq^{d^{+}}$is in fact the ordering deduced from a quasi-metric $d^{+}$on $\mathbf{B}(X, d)$, defined by $d^{+}((x, r),(y, s))=$ $\max (d(x, y)-r+s, 0)$.

The $d$-Scott topology coincides with the familiar open ball topology when $d$ is a metric [16, Proposition 7.4.46], or when $X, d$ is Smyth-complete [16, Proposition 7.4.47]. It coincides with the generalized Scott topology of [3] when $X, d$ is an algebraic complete quasi-metric space [16, Exercise 7.4.69] (see below for the definition of "algebraic complete"). On $\overline{\mathbb{R}}_{+}$, the $\mathrm{d}_{\mathbb{R}}$-Scott topology coincides with the Scott topology of the usual ordering $\leq$ : its non-trivial opens are the open intervals $] r,+\infty], r \in \mathbb{R}_{+}$. The $d^{+}$-Scott topology also coincides with the familiar Scott topology on $\mathbf{B}(X, d)$, see [18, Lemma 3.6] or [16, Exercise 7.4.53].

We say that $X, d$ is complete (or Yoneda-complete) if and only if $\mathbf{B}(X, d)$ is a dcpo. We take this as a definition, although this is really a theorem, due to Kostanek and Waszkiewicz, see [25] and [16, Theorem 7.4.27].

Outside complete spaces, we would like to avoid certain pathologies and we will therefore concentrate on standard quasi-metric spaces [20, Section 2]. $X, d$ is standard if and only if for every $s \in \mathbb{R}_{+}$, a directed family of formal balls $\left(x_{i}, r_{i}\right)_{i \in I}$ has a supremum in $B(X, d)$ if and only if $\left(x_{i}, r_{i}+s\right)_{i \in I}$ has a supremum in $B(X, d)$. Writing the supremum of the former as $(x, r)$, we then have that $r=\inf _{i \in I} r_{i}$, and that the supremum of the latter is $(x, r+s)$ this holds not only for $s \in \mathbb{R}_{+}$, but for every $s \geq-r$. In particular, the radius map $(x, r) \mapsto r$ is Scott-continuous from $\mathbf{B}(X, d)$ to $\overline{\mathbb{R}}_{+}^{o p}\left(\overline{\mathbb{R}}_{+}\right.$with the opposite ordering $\geq)$, and for every $s \in \mathbb{R}_{+}$, the map _ $+s:(x, r) \mapsto(x, r+s)$ is Scott-continuous from $\mathbf{B}(X, d)$ to itself [20, Proposition 2.4]. Moreover, and we will use that fact several times, the set $V_{\epsilon}=\{(x, r) \in \mathbf{B}(X, d) \mid r<\epsilon\}$ is Scott-open for every $\epsilon>0$, whenever $X, d$ is standard. This is because $V_{\epsilon}$ is the inverse image of the Scott-open subset $\left[0, \epsilon\left[\right.\right.$ of $\overline{\mathbb{R}}_{+}^{o p}$ by the radius map.

All complete quasi-metric spaces are standard. Also, all metric spaces are standard. Even posets, which can all be seen as quasi-metric spaces, by defining $d$ as $d_{\leq}$, are all standard [20, Proposition 2.2]. Note that $\overline{\mathbb{R}}_{+}, d_{\mathbb{R}}$ is standard, being complete. 
Directed complete posets (dcpos) come into further varieties: algebraic dcpos, continuous dcpos, notably, and that extends to algebraic posets and continuous posets. The same happens with quasi-metric spaces. We say that $X, d$ is a continuous quasi-metric space if any only if $\mathbf{B}(X, d)$ is a continuous poset. Hence it is continuous complete if and only if $\mathbf{B}(X, d)$ is a continuous dcpo. This is again originally a theorem, not a definition [25].

For the sake of completeness, let us recall what a continuous poset is. The way-below relation $\ll$ on a poset is defined by $x \ll y$ if and only if every directed family that has a supremum above $y$ contains an element above $x$. (We will often say "above" for "larger than or equal to", and similarly for "below".) A basis of a poset is a subset $\mathcal{B}$ such that every point $x$ of the poset is the supremum of a directed family of elements of $\mathcal{B}$. A continuous poset is a poset with a basis. In that case, the poset itself is a basis, namely the largest one.

A point $x \in X$ is a center point if and only if $B_{(x, 0), \epsilon}^{d^{+}}$is Scott-open in $\mathbf{B}(X, d)$ for every $\epsilon>0$. The notion coincides with that of a $d$-finite point, provided that $X, d$ is standard, see Lemma 5.7 of [20]. A standard quasimetric space $X, d$ is algebraic if and only if every point is a $d$-limit of a Cauchy net of $d$-finite points, or equivalently of a Cauchy-weightable net of $d$-finite points - see the comments after Example 5.3 of [20] - or equivalently, that for every $x \in X$, there is a directed family $\left(x_{i}, r_{i}\right)_{i \in I}$ of formal balls such that each $x_{i}$ is a center point and whose supremum is $(x, 0)$. Another equivalent definition is: a standard quasi-metric space $X, d$ is algebraic if and only if the open balls $B_{(x, 0), \epsilon}^{d^{+}}$, where $x$ ranges over the center points and $\epsilon>0$, form a base of the Scott topology on $\mathcal{B}(X, d)$ [20, Definition 5.11, Theorem 5.16]. Every (standard) algebraic quasi-metric space is continuous. Moreover, for every center point $z$, we have $(z, t) \ll(y, s)$ if and only if $d(z, y)<t-s$; more generally, $(y, s) \ll(x, r)$ if and only if there is a center point $z$ and some $t \in \mathbb{R}_{+}$such that $(y, s) \leq^{d^{+}}(z, t)$ and $d(z, x)<t-r[20$, Proposition 5.18].

In a metric space, those complications vanish, as every point is a center point. Every point is a center point in a Smyth-complete space as well. However, one has to be careful with $\overline{\mathbb{R}}_{+}, \mathrm{d}_{\mathbb{R}}$ already, whose center points are the elements of $\mathbb{R}_{+}$, but not $+\infty$. And indeed, the $d_{\mathbb{R}^{-}}$Scott topology coincides with the Scott topology, in which $B_{+\infty,<\epsilon}^{\mathrm{d}_{\mathbb{R}}}=\{+\infty\}$ is not open.

Given a $d$-Scott open subset $U$ of $X$, there is a largest Scott open subset $\widehat{U}$ of $\mathbf{B}(X, d)$ such that $U=\widehat{U} \cap X$. (We silently equate $X$ with a subspace of 
$\mathbf{B}(X, d)$.) The assignment $U \mapsto \widehat{U}$ is monotonic; being left-adjoint to the map $V \mapsto V \cap X$, it preserves arbitrary meets (namely, interiors of intersections), but it satisfies no other remarkable property in general. A quasi-metric space $X, d$ is Lipschitz regular if and only if that assignment is Scott-continuous. This is defined and studied in Section 4 of [18]. If that is the case, then $X, d$ is finitarily embedded in $\mathbf{B}(X, d)$; see [11] for more information on finitary embeddings. For algebraic complete quasi-metric spaces, Lipschitz regularity is equivalent to having relatively compact balls, a stronger requirement than local compactness. This displays Lipschitz regularity as a rather strong requirement. However, the space of formal balls $\mathbf{B}(X, d)$ of any quasi-metric space $X, d$, with the $d^{+}$quasi-metric, is Lipschitz regular [18, Theorem 4.12].

Given a map $f$ from a quasi-metric space $X, d$ to a quasi-metric space $Y, \partial, f$ is $\alpha$-Lipschitz if and only if $\partial(f(x), f(y)) \leq \alpha d(x, y)$ for all $x, y \in X$. (When $\alpha=0$ and $d(x, y)=+\infty$, we take the convention that $0 .+\infty=0$.)

For every $\alpha \in \mathbb{R}_{+}$, and every map $f: X, d \rightarrow Y, \partial$, let $\mathbf{B}^{\alpha}(f) \operatorname{map}(x, r) \in$ $\mathbf{B}(X, d)$ to $(f(x), \alpha r) \in \mathbf{B}(Y, \partial)$. Then $f$ is $\alpha$-Lipschitz if and only if $\mathbf{B}^{\alpha}(f)$ is monotonic.

Contrarily to the case of spaces with the open ball topology, a Lipschitz map need not be continuous. For example, on posets seen as quasi-metric spaces through $d(x, y)=0$ if $x \leq y,+\infty$ otherwise, the Lipschitz maps are the monotonic maps [20, Example 6.1], and the continuous maps, with respect to the underlying $d$-Scott topologies, are the Scott-continuous maps.

We say that a map $f: X, d \rightarrow Y, \partial$ between quasi-metric spaces is $\alpha$ Lipschitz continuous if and only if $\mathbf{B}^{\alpha}(f)$ is Scott-continuous [18, Definition 2.3]. While " $\alpha$-Lipschitz continuous" should be thought of as one epithet, not as the conjunction of " $\alpha$-Lipschitz" and "continuous" in general, we have the following. When both $X, d$ and $Y, \partial$ are standard, $f: X \rightarrow Y$ is $\alpha$-Lipschitz continuous if and only if $f$ is both $\alpha$-Lipschitz, and continuous from $X$ with its $d$-Scott topology to $Y$ with its $\partial$-Scott topology [18, Proposition 2.5].

Given any topological space $X$, a map $h: X \rightarrow \overline{\mathbb{R}}_{+}$is lower semicontinuous if and only if it is continuous from $X$ to $\overline{\mathbb{R}}_{+}$with its Scott topology. We write $\mathcal{L} X$ for the space of all lower semicontinuous maps from $X$ to $\overline{\mathbb{R}}_{+}$, with the Scott topology of the pointwise ordering.

Given any standard quasi-metric space $X, d$, every $\alpha$-Lipschitz continuous map from $X, d$ to $\overline{\mathbb{R}}_{+}, \mathrm{d}_{\mathbb{R}}$ is lower semicontinuous. We write $\mathcal{L}_{\alpha}(X, d)$, or simply $\mathcal{L}_{\alpha} X$, for the subspace of $\mathcal{L} X$ consisting of all $\alpha$-Lipschitz continuous maps. We also write $\mathcal{L}_{\infty}(X, d)$, or $\mathcal{L}_{\infty} X$, for the space of all Lipschitz con- 
tinuous maps, namely $\bigcup_{\alpha \in \mathbb{R}_{+}} \mathcal{L}_{\alpha}(X, d)$, still with the subspace topology from $\mathcal{L} X$.

Given a map $h: X \rightarrow \overline{\mathbb{R}}_{+}$, where $X, d$ is standard, define $h^{\prime}: \mathbf{B}(X, d) \rightarrow$ $\mathbb{R} \cup\{+\infty\}$ by $h^{\prime}(x, r)=h(x)-\alpha r$. Then $h$ is $\alpha$-Lipschitz if and only if $h^{\prime}$ is monotonic, and $h$ is $\alpha$-Lipschitz continuous if and only if $h^{\prime}$ is Scottcontinuous [18, Lemma 2.7].

The Lipschitz continuous maps from a standard quasi-metric space to $\overline{\mathbb{R}}_{+}, \mathrm{d}_{\mathbb{R}}$ enjoy the usual closure properties [20, Proposition 6.7]: if $f$ is $\beta$ Lipschitz continuous then $\alpha f$ is $\alpha \beta$-Lipschitz continuous; if $f$ is $\alpha$-Lipschitz continuous and $g$ is $\beta$-Lipschitz continuous then $f+g$ is $(\alpha+\beta)$-Lipschitz continuous; if $f, g$ are $\alpha$-Lipschitz continuous, then so are $\min (f, g)$ and $\max (f, g)$; if $\left(f_{i}\right)_{i \in I}$ is any family of $\alpha$-Lipschitz continuous maps, then the pointwise supremum $\sup _{i \in I} f_{i}$ is also $\alpha$-Lipschitz continuous; if $\alpha \leq \beta$ and $f$ is $\alpha$-Lipschitz continuous then $f$ is $\beta$-Lipschitz continuous; every constant map is $\alpha$-Lipschitz continuous. Moreover, if $f: X \rightarrow Y$ is $\alpha$-Lipschitz continuous and $g: Y \rightarrow Z$ is $\beta$-Lipschitz continuous, then $g \circ f$ is $\alpha \beta$-Lipschitz continuous [18, Lemma 2.9].

\subsection{Continuous valuations}

A valuation on a topological space $X$ is a map $\nu: \mathcal{O} X \rightarrow \overline{\mathbb{R}}_{+}$, where $\mathcal{O} X$ is the lattice of open subsets of $X$, satisfying the following laws: $\nu(\emptyset)=0$ (strictness), $U \subseteq V$ implies $\nu(U) \leq \nu(V)$ (monotonicity), $\nu(U)+\nu(V)=$ $\nu(U \cup V)+\nu(U \cap V)$ (modularity). A continuous valuation is additionally Scott-continuous, namely: for every directed family $\left(U_{i}\right)_{i \in I}$ of open subsets of $X, \nu\left(\bigcup_{i \in I} U_{i}\right)=\sup _{i \in I} \nu\left(U_{i}\right)[21,22]$.

A subprobability (resp., probability) valuation is a continuous valuation $\nu$ such that $\nu(X) \leq 1$ (resp., $\nu(X)=1$ ). A valuation is bounded if and only if $\nu(X)<+\infty$.

A $\tau$-smooth measure on a topological space $X$ is, by definition, a measure whose restriction to $\mathcal{O} X$ is Scott-continuous. Hence every $\tau$-smooth measure on $X$ yields a continuous valuation by restricting it to $\mathcal{O} X$. Conversely, every continuous valuation $\nu$ extends to a (necessarily $\tau$-smooth) measure on all Borel sets of any LCS-complete space $X$ [7, Theorem 1.1]. An LCS-complete space is a space homeomorphic to a $G_{\delta}$ subset of a locally compact sober space. That extension is unique if $\nu$ is bounded, see [7, Lemma 18.1] for example.

All continuous complete quasi-metric spaces are LCS-complete in their $d$-Scott topology [7, Theorem 4.1]). The continuous complete quasi-metric 
spaces will be the most important class of quasi-metric spaces we consider in this paper. In this setting, one should then remember that bounded continuous valuations and bounded $\tau$-smooth measures are in bijective correspondence, and can be considered to be equivalent notions. This also applies to the case of continuous dcpos, which are locally compact and sober, hence also LCS-complete.

One should also note that every measure on a hereditarily Lindelöf space, in particular on a second-countable space, is $\tau$-smooth [1, Theorem 3.1]. It follows that, for second-countable LCS-complete spaces (which happen to be M. de Brecht's quasi-Polish spaces [6], and which include all Polish spaces), there is a bijection between bounded continuous valuations and bounded measures. This shows how close the two notions are.

There is a notion of integral $\int_{x \in X} h(x) d \nu$ of any lower semicontinuous map $h$ with respect to any continuous valuation $\nu$, which one may define by using the Choquet formula $\left.\left.\int_{0}^{+\infty} \nu\left(h^{-1}(] t,+\infty\right]\right)\right) d t$. That integral is linear in both $h$ and $\nu$, meaning that it commutes with non-negatively weighted finite sums, and it is Scott-continuous also both in $h$ and $\nu$. The Choquet formula is of course due to Choquet [4, Chapter VII, Section 48.1], and its use in the theory of continuous valuations is due to Tix [30].

Given a continuous valuation $\nu$ on a space $X$, and a continuous map $f: X \rightarrow Y$, the push-forward $f[\nu]$ is the continuous valuation defined by $f[\nu](V)=\nu\left(f^{-1}(V)\right)$. The following change of variable formula holds:

$$
\int_{y \in Y} h(y) d f[\nu]=\int_{x \in X} h(f(x)) d \nu .
$$

This is a simple consequence of the Choquet formula.

A prevision is a Scott-continuous map $G: \mathcal{L} X \rightarrow \overline{\mathbb{R}}_{+}$that is positively homogeneous, namely such that $G(a h)=a G(h)$ for all $a \in \mathbb{R}_{+}$and $h \in$ $\mathcal{L} X$. A prevision $G$ is sublinear, resp. superlinear, resp. linear if and only if it satisfies the law $G\left(h+h^{\prime}\right) \leq G(h)+G\left(h^{\prime}\right)$ (resp., $\geq$, resp. =). Given a continuous valuation $\nu$, the map $h \mapsto \int_{x \in X} h(x) d \nu$ is a linear prevision. Conversely, given any linear prevision $G$, the map $U \mapsto G\left(\chi_{U}\right)$, where $\chi_{U}$ is the characteristic function of the open set $U$, is a continuous valuation. Moreover, the two operations are inverse of each other.

A prevision $G$ is subnormalized (resp., normalized) if and only if $G(\alpha .1+$ $h) \leq \alpha+G(h)$ holds (resp., $=$ ) for all $\alpha \in \mathbb{R}_{+}$and $h \in \mathcal{L} X$, where $\mathbf{1}$ is the constant 1 map. The above isomorphism cuts down to one between subprobability continuous valuations and and subnormalized linear previsions, and 
between probability continuous valuations and normalized linear previsions. We note that, for a linear prevision $G, G$ is subnormalized if and only if $G(\mathbf{1}) \leq 1$, and normalized if and only if $G(\mathbf{1})=1$.

We write $\mathbf{V} X$ for the set of all continuous valuations on $X, \mathbf{V}_{\leq 1} X$ for the subset of all subprobability valuations and $\mathbf{V}_{1} X$ for the set of all probability valuations. We will later use quasi-metrics $d_{\mathrm{KR}}$ and $d_{\mathrm{KR}}^{a}$ on those sets, and we will therefore consider the $d_{\mathrm{KR}}$-Scott and $d_{\mathrm{KR}}^{a}$-Scott topologies. In the meantime, a natural topology on those spaces is the so-called weak topology (short for "Scott weak* topology"), with subbasic open sets $[h>b]$ defined as the set of those $\nu$ such that $\int_{x \in X} h(x) d \nu>b, h \in \mathcal{L} X, b \in \mathbb{R}_{+}$.

One should observe that those spaces are also dcpos under the stochastic ordering $(\mu \leq \nu$ if and only if $\mu(U) \leq \nu(U)$ for every open subset $U$ of $X)$, and that $\mathbf{V} X$ and $\mathbf{V}_{\leq 1} X$ are even continuous depos if $X$ is a continuous depo [13, Theorem IV-9.16]. That result is an extension of a theorem by C. Jones that shows a similar result for $\mathbf{V}_{\leq 1} Y$ [21, Corollary 5.4].

\section{The Kantorovich-Rubinstein quasi-metric on continuous valua- tions}

We define the Kantorovich-Rubinstein quasi-metric $d_{\mathrm{KR}}$, and its bounded variant $d_{\mathrm{KR}}^{a}$, and we study some of their basic properties. We will also explore alternative, equivalent definitions. Our basic quasi-metric is the following.

Definition $3.1\left(d_{\mathbf{K R}}\right)$. Let $X, d$ be a quasi-metric space. The KantorovichRubinstein quasi-metric (for short, the $\mathrm{KRH}$ quasi-metric) on $\mathbf{V X}$ is defined by:

$$
d_{K R}\left(\nu, \nu^{\prime}\right)=\sup _{h \in \mathcal{L}_{1} X} \mathrm{~d}_{\mathbb{R}}\left(\int_{x \in X} h(x) d \nu, \int_{x \in X} h(x) d \nu^{\prime}\right) .
$$

In general, on any space of previsions on $X$, we define:

$$
d_{K R}\left(F, F^{\prime}\right)=\sup _{h \in \mathcal{L}_{1} X} \mathrm{~d}_{\mathbb{R}}\left(F(h), F^{\prime}(h)\right) .
$$

This is a quasi-metric on any standard quasi-metric space, as remarked at the end of Section 6 of [20]. We give a slightly expanded argument in Lemma 3.7 below. 
Remark 3.2. The name of that quasi-metric stems from analogous definitions of metrics on spaces of measures. The classical Kantorovich-Rubinstein metric between two bounded measures $\mu$ and $\mu^{\prime}$ is given as:

$$
\sup _{h}\left|\int_{x \in X} h(x) d \mu-\int_{x \in X} h(x) d \mu^{\prime}\right|,
$$

where $h$ ranges over the set $L_{1}(X)$ of 1-Lipschitz maps from $X$ to $\overline{\mathbb{R}}_{+}$, and, depending on authors, is sometimes also constrained to be below $\mathbf{1}$. (We introduce the corresponding variant of $d_{K R}$, where $h$ has to be bounded from above by some constant, in Definition 3.4 below.) This formula can be rewritten as $\sup _{h} \mathrm{~d}_{\mathbb{R}}^{\text {sym }}\left(F(h), F^{\prime}(h)\right)$. In the case of metric (as opposed to quasi-metric) spaces, we shall see in Lemma 7.1 that the use of the symmetrized metric $\mathrm{d}_{\mathbb{R}}^{\text {sym }}$ is irrelevant, and the distance between $\mu$ and $\mu^{\prime}$ is equal to $\sup _{h} \mathrm{~d}_{\mathbb{R}}\left(\int_{x \in X} h(x) d \nu, \int_{x \in X} h(x) d \nu^{\prime}\right)$. Additionally, on a metric space, all 1-Lipschitz maps $h$ are automatically continuous.

Remark 3.3. Let $X$ be a poset, with ordering $\leq$. We see it as a quasi-metric space under the associated $\{0,+\infty\}$-valued quasi-metric $d_{\leq}$. Then $X, d_{\leq}$is standard. For every map $h: X \rightarrow \overline{\mathbb{R}}_{+}, h$ is $\alpha$-Lipschitz (for any $\alpha>0$ ) if and only if it is monotonic: indeed $h(x) \leq h(y)+\alpha d_{\leq}(x, y)$ (for all $x, y \in X$ ) is equivalent to $h(x) \leq h(y)$ (for all $x \leq y$ in $X$ ). The $d_{\leq-S c o t t ~ t o p o l o g y}$ coincides with the Scott topology on $X$ [20, Example 1.8], so the $\alpha$-Lipschitz continuous maps from $X, d_{\leq}$to $\overline{\mathbb{R}}_{+}, \mathrm{d}_{\mathbb{R}}$ are exactly the Scott-continuous maps from $X$ to $\overline{\mathbb{R}}_{+}$.

In this case, we claim that $d_{K R}$ is also $\{0,+\infty\}$-valued on $\mathbf{V X}$. Indeed, either $\nu \leq \nu^{\prime}$ and $d_{K R}\left(\nu, \nu^{\prime}\right)=0$, as can be checked directly (or see Lemma 3.7 below), or there is an open set $U$ such that $\nu(U)>\nu^{\prime}(U)$. In that case, for every $r \in \mathbb{R}_{+}, r \chi_{U}$ is Scott-continuous, hence 1-Lipschitz continuous, so that $d_{K R}\left(\nu, \nu^{\prime}\right) \geq \mathbf{d}_{\mathbb{R}}\left(r \nu(U), r \nu^{\prime}(U)\right)=r\left(\nu(U)-\nu^{\prime}(U)\right)$. Since $r$ is arbitrary large, $d_{K R}\left(\nu, \nu^{\prime}\right)=+\infty$. We have in fact shown that $d_{K R}\left(\nu, \nu^{\prime}\right)$ is equal to 0 if $\nu \leq \nu^{\prime}$, to $+\infty$ otherwise, so $d_{K R}$ is the quasi-metric associated with the stochastic ordering on $\mathbf{V X}$ (provided that $X$ is a poset).

Definition $3.4\left(\mathcal{L}_{\alpha}^{a}(X, d), d_{\mathbf{K R}}^{a}\right)$. Let $X, d$ be a quasi-metric space, and $a \in$ $\mathbb{R}_{+}, a>0$. Let $\mathcal{L}_{\alpha}^{a}(X, d)$, or $\mathcal{L}_{\alpha}^{a} X$, be the subspace of those $\alpha$-Lipschitz continuous maps from $X, d$ to $\overline{\mathbb{R}}_{+}$with values in $[0, \alpha a]$. 
The a-bounded Kantorovich-Rubinstein quasi-metric on $\mathrm{VX}$ is defined by:

$$
d_{K R}^{a}\left(\nu, \nu^{\prime}\right)=\sup _{h \in \mathcal{L}_{1}^{a} X} \mathrm{~d}_{\mathbb{R}}\left(\int_{x \in X} h(x) d \nu, \int_{x \in X} h(x) d \nu^{\prime}\right) .
$$

In general, on any space of previsions on $X$, we define:

$$
d_{K R}^{a}\left(F, F^{\prime}\right)=\sup _{h \in \mathcal{L}_{1}^{a} X} \mathrm{~d}_{\mathbb{R}}\left(F(h), F^{\prime}(h)\right) .
$$

The $d_{\mathrm{KR}}$ quasi-metric is interesting mostly on subspaces of subprobability, resp. probability valuations, as we explain now.

Remark 3.5. For any two continuous valuations $\nu$ and $\nu^{\prime}$ such that $\nu(X)>$ $\nu^{\prime}(X), d_{K R}\left(\nu, \nu^{\prime}\right)=+\infty$. Indeed, let $h=a .1, a \in \mathbb{R}_{+}$: the right-hand side of (1) is larger than or equal to $a\left(\nu(X)-\nu^{\prime}(X)\right)$ for every a, hence equal to $+\infty$, by taking suprema over a. This incongruity disappears on the subspace $\mathbf{V}_{1} X$ of probability valuations.

Remark 3.6. Following up on Remark 3.3, we evaluate $d_{K R}^{a}$ on $\mathbf{V} X$ when $X$ is a poset. The result is very different: $d_{K R}^{a}\left(\nu, \nu^{\prime}\right)$ is indeed equal to $a \cdot \sup _{U \in \mathcal{O} X} \mathrm{~d}_{\mathbb{R}}\left(\nu(U), \nu^{\prime}(U)\right)$. When $a=1$, this is the sup quasi-metric considered by Sünderhauf [29].

This is proved as follows. From the first part of Remark 3.3, the maps $h \in \mathcal{L}_{1}^{a} X$ are the Scott-continuous maps from $X$ to $\overline{\mathbb{R}}_{+}$that are bounded by a. We first verify that $d_{K R}^{a}\left(\nu, \nu^{\prime}\right) \leq a \cdot \sup _{U \in \mathcal{O} X} \mathrm{~d}_{\mathbb{R}}\left(\nu(U), \nu^{\prime}(U)\right)$. We use the Choquet formula: $\left.d_{K R}^{a}\left(\nu, \nu^{\prime}\right)=\sup _{h \in \mathcal{L}_{1}^{a}(X, d)} d_{\mathbb{R}}\left(\int_{0}^{+\infty} \nu\left(h^{-1}(] t,+\infty\right]\right)\right) d t$, $\left.\left.\left.\int_{0}^{+\infty} \nu^{\prime}\left(h^{-1}(] t,+\infty\right]\right)\right) d t\right)$, and that is equal to $\left.\sup _{h \in \mathcal{L}_{1}^{a}(X, d)} \mathrm{d}_{\mathbb{R}}\left(\int_{0}^{a} \nu\left(h^{-1}(] t,+\infty\right]\right)\right) d t$, $\left.\left.\left.\int_{0}^{a} \nu^{\prime}\left(h^{-1}(] t,+\infty\right]\right)\right) d t\right)$ since $\left.\left.h^{-1}(] t,+\infty\right]\right)$ is empty for every $t \geq a$, for every $h \in \mathcal{L}_{1}^{a}(X, d)$. If there is an $h \in \mathcal{L}_{1}^{a}(X, d)$ such that $\left.\left.\int_{0}^{a} \nu\left(h^{-1}(] t,+\infty\right]\right)\right) d t=$ $+\infty$ but $\left.\left.\int_{0}^{a} \nu^{\prime}\left(h^{-1}(] t,+\infty\right]\right)\right) d t<+\infty$, then it is easy to see that there is an open set $U$ of the form $\left.\left.h^{-1}(] t,+\infty\right]\right)$ such that $\nu(U)=+\infty$, and necessarily $\nu^{\prime}(U)<+\infty$, so a. $\sup _{U \in \mathcal{O} X} \mathrm{~d}_{\mathbb{R}}\left(\nu(U), \nu^{\prime}(U)\right)=+\infty$, and therefore the inequality is trivial. Otherwise, for every $h \in \mathcal{L}_{1}^{a}(X, d)$, either $\left.\left.\left.\left.\int_{0}^{a} \nu\left(h^{-1}(] t,+\infty\right]\right)\right) d t=\int_{0}^{a} \nu^{\prime}\left(h^{-1}(] t,+\infty\right]\right)\right) d t=+\infty$, in which case we have $\left.\left.\left.\left.\mathrm{d}_{\mathbb{R}}\left(\int_{0}^{a} \nu\left(h^{-1}(] t,+\infty\right]\right)\right) d t, \int_{0}^{a} \nu^{\prime}\left(h^{-1}(] t,+\infty\right]\right)\right) d t\right)=0$, or $\left.\left.\int_{0}^{a} \nu\left(h^{-1}(] t,+\infty\right]\right)\right) d t<$ $+\infty$, in which case $\left.\left.\left.\left.\mathrm{d}_{\mathbb{R}}\left(\int_{0}^{a} \nu\left(h^{-1}(] t,+\infty\right]\right)\right) d t, \int_{0}^{a} \nu^{\prime}\left(h^{-1}(] t,+\infty\right]\right)\right) d t\right)$ is equal to $\left.\left.\left.\left.\max \left(\int_{0}^{a}\left(\nu\left(h^{-1}(] t,+\infty\right]\right)\right)-\nu^{\prime}\left(h^{-1}(] t,+\infty\right]\right)\right)\right) d t, 0\right)$; since $\left.\left.\nu\left(h^{-1}(] t,+\infty\right]\right)\right)-$ $\left.\nu^{\prime}\left(h^{-1}(] t,+\infty\right]\right) \leq \sup _{U \in \mathcal{O} X} \mathrm{~d}_{\mathbb{R}}\left(\nu(U), \nu^{\prime}(U)\right)$, the inequality follows. In the 
converse direction, the map a $\chi_{U}$ is Scott-continuous, hence in $\mathcal{L}_{1}^{a}(X, d)$ for every $U \in \mathcal{O} X$, so a. $\sup _{U \in \mathcal{O} X} \mathrm{~d}_{\mathbb{R}}\left(\nu(U), \nu^{\prime}(U)\right) \leq d_{K R}^{a}\left(\nu, \nu^{\prime}\right)$.

For the next lemma, we need to know that for every map $f: X \rightarrow \overline{\mathbb{R}}_{+}$ from a standard quasi-metric space $X, d$ to $\overline{\mathbb{R}}_{+}$, there is a largest $\alpha$-Lipschitz continuous map $f^{(\alpha)}$ below $f$. When $f$ is lower semicontinuous, the family $\left(f^{(\alpha)}\right)_{\alpha \in \mathbb{R}_{+}}$is a chain, and $\sup _{\alpha \in \mathbb{R}_{+}} f^{(\alpha)}=f$, where suprema are taken pointwise [20, Theorem 6.17].

Lemma 3.7. Let $X, d$ be a standard quasi-metric space, and $a \in \mathbb{R}_{+} \backslash\{0\}$. For all previsions $F, F^{\prime}$ on $X$, the following are equivalent: $(a) F \leq F^{\prime}$; (b) $d_{K R}\left(F, F^{\prime}\right)=0 ;(c) d_{K R}^{a}\left(F, F^{\prime}\right)=0$. In particular, $d_{K R}$ and $d_{K R}^{a}$ are quasi-metrics.

Proof. $(a) \Rightarrow(b)$. For every $h \in \mathcal{L}_{1}(X, d), F(h) \leq F^{\prime}(h)$, so $d_{\mathbb{R}}\left(F(h), F^{\prime}(h)\right)=$ 0 .

$(b) \Rightarrow(c)$. If $d_{\mathrm{KR}}\left(F, F^{\prime}\right)=0$, then $F(h) \leq F^{\prime}(h)$ for every $h \in \mathcal{L}_{1}(X, d)$, hence also for every $h \in \mathcal{L}_{1}^{a}(X, d)$.

$(c) \Rightarrow(a)$. Let $f$ be an arbitrary element of $\mathcal{L} X$. For every $\alpha>0$, $1 / \alpha \min \left(a \alpha, f^{(\alpha)}\right)$ is in $\mathcal{L}_{1}^{a}(X, d)$, so $F\left(1 / \alpha \min \left(a \alpha, f^{(\alpha)}\right)\right) \leq F^{\prime}\left(1 / \alpha \min \left(a \alpha, f^{(\alpha)}\right)\right)$. Multiply by $\alpha: F\left(\min \left(a \alpha, f^{(\alpha)}\right)\right) \leq F^{\prime}\left(\min \left(a \alpha, f^{(\alpha)}\right)\right)$. The family $\left(f^{(\alpha)}\right)_{\alpha>0}$, hence also the family $\left(\min \left(a \alpha, f^{(\alpha)}\right)\right)_{\alpha>0}$, is a chain whose supremum is $f$. Using the fact that $F$ and $F^{\prime}$ are Scott-continuous, $F(f) \leq F^{\prime}(f)$, and as $f$ is arbitrary, $(a)$ follows.

This allows us to show that if $d_{\mathrm{KR}}\left(F, F^{\prime}\right)=d_{\mathrm{KR}}\left(F^{\prime}, F\right)=0$ then $F=F^{\prime}$ (and similarly with $d_{\mathrm{KR}}^{a}$ ), which is the only non-trivial axiom of quasi-metrics we have to verify.

The following shows that we can restrict to bounded maps $h \in \mathcal{L}_{1}(X, d)$. A map $h$ is bounded if and only if there is a constant $a \in \mathbb{R}_{+}$such that for every $x \in X, h(x) \leq a$.

Lemma 3.8. Let $X, d$ be a quasi-metric space. For all previsions $F, F^{\prime}$ on $X$,

$$
d_{K R}\left(F, F^{\prime}\right)=\sup _{h \text { bounded } \in \mathcal{L}_{1} X} \mathrm{~d}_{\mathbb{R}}\left(F(h), F^{\prime}(h)\right) .
$$

Proof. For every $h \in \mathcal{L}_{1}(X, d), h$ is the pointwise supremum of the chain $(\min (h, a))_{a \in \mathbb{R}_{+}}$, where $\min (h, a): x \mapsto \min (h(x), a)$. The right-hand side of the claimed inequality is clearly less than or equal to $d_{\mathrm{KR}}\left(F, F^{\prime}\right)$. In the 
converse direction, it suffices to show that for every $r \in \mathbb{R}_{+}$such that $r<$ $d_{\mathrm{KR}}\left(F, F^{\prime}\right)$, there is a bounded map $h^{\prime} \in \mathcal{L}_{1}(X, d)$ such that $r<\mathrm{d}_{\mathbb{R}}\left(F\left(h^{\prime}\right)\right.$, $\left.F^{\prime}\left(h^{\prime}\right)\right)$. Since $r<d_{\mathrm{KR}}(F, F)$, there is an $h \in \mathcal{L}_{1}(X, d)$ such that $r<\mathrm{d}_{\mathbb{R}}(F(h)$, $\left.F^{\prime}(h)\right)$. This implies that $F(h)>F^{\prime}(h)+r$, a fact that one verifies easily by going through the three cases of the definition of $d_{\mathbb{R}}$.

Since $F$ is Scott-continuous, there is an $a \in \mathbb{R}_{+}$such that $F(\min (h(x), a))>$ $F^{\prime}(h)+r \geq F^{\prime}(\min (h(x), a))+r$, so we can take $h^{\prime}=\min (h, a)$.

We obtain the following relation between the $d_{\mathrm{KR}}$ and $d_{\mathrm{KR}}^{a}$ quasi-metrics.

Lemma 3.9. Let $X, d$ be a quasi-metric space. Order quasi-metrics on any space of previsions pointwise. Then $\left(d_{K R}^{a}\right)_{a \in \mathbb{R}_{+}, a>0}$ is a chain, and for all previsions $F, F^{\prime}, d_{K R}\left(F, F^{\prime}\right)=\sup _{a \in \mathbb{R}_{+}, a>0} d_{K R}^{a}\left(F, F^{\prime}\right)$.

Proof. Clearly, for $0<a \leq a^{\prime}, \mathcal{L}_{1}^{a}(X, d)$ is included in $\mathcal{L}_{1}^{a^{\prime}}(X, d)$, so $d_{\mathrm{KR}}^{a}\left(F, F^{\prime}\right) \leq d_{\mathrm{KR}}^{a^{\prime}}\left(F, F^{\prime}\right)$. Therefore $\left(d_{\mathrm{KR}}^{a}\right)_{a \in \mathbb{R}_{+}, a>0}$ is a chain. Similarly, $d_{\mathrm{KR}}^{a}\left(F, F^{\prime}\right) \leq d_{\mathrm{KR}}\left(F, F^{\prime}\right)$. Finally, the supremum $\sup _{a \in \mathbb{R}_{+}, a>0} d_{\mathrm{KR}}^{a}\left(F, F^{\prime}\right)$ is equal to $\sup _{a \in \mathbb{R}_{+}, a>0, h \in \mathcal{L}_{1}^{a}(X, d)} \mathrm{d}_{\mathbb{R}}\left(F(h), F^{\prime}(h)\right)=\sup _{h \text { bounded } \in \mathcal{L}_{1}(X, d)} \mathrm{d}_{\mathbb{R}}\left(F(h), F^{\prime}(h)\right)$, and that is equal to $d_{\mathrm{KR}}\left(F, F^{\prime}\right)$ by Lemma 3.8.

The following characterizes the ordering on $\mathbf{B}\left(\mathbf{V} X, d_{\mathrm{KR}}\right)$ and on $\mathbf{B}\left(\mathbf{V} X, d_{\mathrm{KR}}^{a}\right)$, and more generally on $\mathbf{B}\left(Y, d_{\mathrm{KR}}\right)$ and on $\mathbf{B}\left(Y, d_{\mathrm{KR}}^{a}\right)$, where $Y$ is any space of previsions on $X$.

Lemma 3.10. Let $X, d$ be a standard quasi-metric space, let $F, F^{\prime}$ be two previsions on $X$, and let $r, r^{\prime}$ be two elements of $\mathbb{R}_{+}$. The following are equivalent:

1. $(F, r) \leq d_{K R}^{+}\left(F^{\prime}, r^{\prime}\right)$;

2. $r \geq r^{\prime}$ and, for every $h \in \mathcal{L}_{1}(X, d), F(h)-r \leq F^{\prime}(h)-r^{\prime}$;

3. $r \geq r^{\prime}$ and, for every $h \in \mathcal{L}_{\alpha}(X, d), \alpha>0, F(h)-\alpha r \leq F^{\prime}(h)-\alpha r^{\prime}$.

Given $a \in \mathbb{R}_{+} \backslash\{0\}$, the same equivalence holds with $d_{K R}$ replaced by $d_{K R}^{a}$, $\mathcal{L}_{1}(X, d)$ by $\mathcal{L}_{1}^{a}(X, d)$ and $\mathcal{L}_{\alpha}(X, d)$ by $\mathcal{L}_{\alpha}^{a}(X, d)$ throughout.

Proof. We only give the proof for $d_{\mathrm{KR}}$, as the case of $d_{\mathrm{KR}}^{a}$ is identical.

$1 \Rightarrow 2$. If $(F, r) \leq d_{\mathrm{KR}}^{+}\left(F^{\prime}, r^{\prime}\right)$, then for every $h \in \mathcal{L}_{1}(X, d), \mathrm{d}_{\mathbb{R}}(F(h)$, $\left.F^{\prime}(h)\right) \leq r-r^{\prime}$. This implies that $r \geq r^{\prime}$, on the one hand, and on the other hand that either $F(h)=F^{\prime}(h)=+\infty$ or $F(h) \neq+\infty$ and $F(h)-F^{\prime}(h) \leq$ $r-r^{\prime}$; in both cases, $F(h)-r \leq F^{\prime}(h)-r^{\prime}$. 
$1 \Rightarrow 3$. For every $h \in \mathcal{L}_{\alpha}(X, d), \alpha>0,1 / \alpha h$ is in $\mathcal{L}_{1}(X, d)$, so item 2 implies $F(1 / \alpha h)-r \leq F^{\prime}(1 / \alpha h)-r^{\prime}$. Multiplying by $\alpha$, and using positive homogeneity, we obtain item 3 .

$3 \Rightarrow 1$. For $\alpha=1$, we obtain that for every $h \in \mathcal{L}_{1}(X, d), F(h)-$ $r \leq F^{\prime}(h)-r^{\prime}$. If $F^{\prime}(h)=+\infty$, then $\mathrm{d}_{\mathbb{R}}\left(F(h), F^{\prime}(h)\right)=0 \leq r-r^{\prime}$. If $F^{\prime}(h) \neq+\infty$, then $F(h)$ cannot be equal to $+\infty$, so $\mathrm{d}_{\mathbb{R}}\left(F(h), F^{\prime}(h)\right)=$ $\max \left(F(h)-F^{\prime}(h), 0\right) \leq \max \left(r-r^{\prime}, 0\right)=r-r^{\prime}$.

Because the definition of $d_{\mathrm{KR}}$ only involves Lipschitz continuous maps, we inquire whether continuous valuations, or rather the associated linear prevision, can be recovered from its action on just $\mathcal{L}_{\infty}(X, d)$.

Definition 3.11 ( $\mathcal{L}$-prevision). Let $X, d$ be a quasi-metric space. An $\mathcal{L}$ prevision on $X$ is any continuous map $G$ from $\mathcal{L}_{\infty}(X, d)$ to $\overline{\mathbb{R}}_{+}$such that $G(\alpha h)=\alpha G(h)$ for all $\alpha \in \mathbb{R}_{+}$and $h \in \mathcal{L}_{\infty}(X, d)$.

The notions of sublinearity, superlinearity, linearity, subnormalization, and normalization, carry over to $\mathcal{L}$-previsions, taking care to quantify over $\mathcal{L}_{\infty}(X, d)$ instead of $\mathcal{L} X$. For a linear $\mathcal{L}$-prevision $G, G$ is subnormalized (resp., normalized) if and only if $G(\mathbf{1}) \leq 1$ (resp., =).

Every continuous valuation $\nu$ defines a linear prevision $h \in \mathcal{L} X \mapsto$ $\int_{x \in X} h(x) d \nu$, and by restricting it to $\mathcal{L}_{\infty} X$, we obtain a linear $\mathcal{L}$-prevision. Conversely, for every $\mathcal{L}$-prevision $G$, let $\bar{G}(h)=\sup _{\alpha \in \mathbb{R}_{+}} G\left(h^{(\alpha)}\right)$. We will show that restriction and $G \mapsto \bar{G}$ are inverse of each other, and we first need the following.

Lemma 3.12. Let $X, d$ be a standard quasi-metric space. For every map $h: X \rightarrow \overline{\mathbb{R}}_{+}$, for all $a, \alpha \in \mathbb{R}_{+}, a h^{(\alpha)}=(a h)^{(a \alpha)}$.

Proof. We have $a h^{(\alpha)} \leq(a h)^{(a \alpha)}$, because $a h^{(\alpha)}$ is $a \alpha$-Lipschitz continuous, below $a h$, and because $(a h)^{(a \alpha)}$ is the largest $a \alpha$-Lipschitz continuous function below $a h$. When $a>0$, by the same argument, $1 / a(a h)^{(a \alpha)} \leq h^{(\alpha)}$, so $a h^{(\alpha)}=(a h)^{(a \alpha)}$. The same equality holds, trivially, when $a=0$.

Proposition 3.13. Let $X, d$ be a standard quasi-metric space.

1. For every $G \in \mathcal{L} \mathbf{P} X, \bar{G}$ is a prevision.

2. The maps $G \in \mathcal{L} \mathbf{P} X \mapsto \bar{G} \in \mathbf{P} X$ and $F \in \mathbf{P} X \mapsto F_{\mid \mathcal{L}_{\infty} X} \in \mathcal{L} \mathbf{P} X$ are inverse of each other. 
3. If $G$ is sublinear, resp. superlinear, resp. linear, resp. subnormalized, resp. normalized, then so is $\bar{G}$.

4. Conversely, if $\bar{G}$ is sublinear, resp. superlinear, resp. linear, resp. subnormalized, resp. normalized, then so is $G$.

Proof. 1. We must first show that $\bar{G}$ is Scott-continuous, i.e., continuous from $\mathcal{L} X$ to $\overline{\mathbb{R}}_{+}$, both with their Scott topologies. Let $h \in \mathcal{L} X$ such that $\bar{G}(h)>a$. Since $h$ is the supremum of the chain $\left(h^{(\alpha)}\right)_{\alpha \in \mathbb{R}_{+}}$, there is an $\alpha \in$ $\mathbb{R}_{+}$such that $G\left(h^{(\alpha)}\right)>a$. Therefore $h^{(\alpha)}$ is in the open subset $\left.\left.G^{-1}(] a,+\infty\right]\right)$ of $\mathcal{L}_{\infty} X$. By definition of a subspace topology, there is a (Scott-)open subset $W$ of $\mathcal{L} X$ such that $\left.\left.G^{-1}(] a,+\infty\right]\right)=W \cap \mathcal{L}_{\infty} X$. Then $h^{(\alpha)}$ is in $W$, and since $h \geq h^{(\alpha)}, h$ is also in $W$. Moreover, for every $g \in W, g^{(\beta)}$ is in $W$ for some $\beta \in \mathbb{R}_{+}$, hence in $\left.\left.G^{-1}(] a,+\infty\right]\right)$. It follows that $G\left(g^{(\beta)}\right)>a$, and therefore $\bar{G}(g)>a$ : hence $W$ is an open neighborhood of $h$ contained in $\left.\left.\bar{G}^{-1}(] a,+\infty\right]\right)$. We conclude that the latter is open in $\mathcal{L} X$, which implies that $\bar{G}$ is continuous.

In order to show that $\bar{G}$ is positively homogeneous, if $a>0$, then $\bar{G}(a h)=\sup _{\beta \in \mathbb{R}_{+}} G\left((a h)^{(\beta)}\right)=\sup _{\alpha \in \mathbb{R}_{+}} G\left((a h)^{(a \alpha)}\right)=\sup _{\alpha \in \mathbb{R}_{+}} G\left(a h^{(\alpha)}\right)$ (by Lemma 3.12) $=a \bar{G}(h)$. When $a=0, \bar{G}(0)=0$. Therefore $\bar{G}$ is a prevision.

2. For every $G \in \mathcal{L} \mathbf{P} X$, the restriction of $\bar{G}$ to $\mathcal{L}_{\infty} X$ maps every Lipschitz (say, $\alpha$-Lipschitz) continuous map $g: X \rightarrow \overline{\mathbb{R}}_{+}$to $\sup _{\beta \in \mathbb{R}_{+}} G\left(g^{(\beta)}\right)$. For every $\beta \geq \alpha, g$ is $\beta$-Lipschitz continuous, and $g^{(\beta)}$ is the largest $\beta$-Lipschitz continuous map below $g$, so $g^{(\beta)}=g$. It follows that $\sup _{\beta \in \mathbb{R}_{+}} G\left(g^{(\beta)}\right)=G(g)$, showing that $\bar{G}_{\mid \mathcal{L}_{\infty} X}=G$.

Note that this says that $G$ and $\bar{G}$ coincide on Lipschitz continuous maps, a fact that we will use several times below.

In the converse direction, for every $F \in \mathbf{P} X, \overline{\left(F_{\mid \mathcal{L}_{\infty} X}\right)}$ maps every function $h \in \mathcal{L} X$ to $\sup _{\alpha \in \mathbb{R}_{+}} F\left(h^{(\alpha)}\right)=F$, because $F$ is Scott-continuous and $\sup _{\alpha \in \mathbb{R}_{+}} h^{(\alpha)}=h$.

3. For all $g, h \in \mathcal{L} X$, for all $\alpha, \beta \in \mathbb{R}_{+}, g^{(\alpha)}+h^{(\beta)} \leq(g+h)^{(\alpha+\beta)}$, because the left-hand side is an $(\alpha+\beta)$-Lipschitz continuous map below $g+h$, and the right-hand side is the largest. If $G$ is superlinear, it follows that $\bar{G}(g)+\bar{G}(h)=\sup _{\alpha, \beta \in \mathbb{R}_{+}} G\left(g^{(\alpha)}\right)+G\left(h^{(\beta)}\right) \leq \sup _{\alpha, \beta \in \mathbb{R}_{+}} G\left(g^{(\alpha)}+h^{(\beta)}\right) \leq$ $\sup _{\alpha, \beta \in \mathbb{R}_{+}} G\left((g+h)^{(\alpha+\beta)}\right)=\bar{G}(g+h)$, hence that $\bar{G}$ is superlinear, too.

If $G$ is sublinear, then we need another argument. We wish to show that $\bar{G}(g+h) \leq \bar{G}(g)+\bar{G}(h)$. To this end, let $a$ be an arbitrary element of 
$\mathbb{R}_{+}$such that $a<\bar{G}(g+h)$. Since $\bar{G}$ is Scott-continuous (item 1 above), $g+h$ is in the Scott-open set $\left.\left.\bar{G}^{-1}(] a,+\infty\right]\right)$. Now $g+h=\sup _{\alpha \in \mathbb{R}_{+}} g^{(\alpha)}+$ $\sup _{\alpha \in \mathbb{R}_{+}} h^{(\alpha)}=\sup _{\alpha \in \mathbb{R}_{+}}\left(g^{(\alpha)}+h^{(\alpha)}\right)$, since addition is Scott-continuous on $\overline{\mathbb{R}}_{+}$, so $g^{(\alpha)}+h^{(\alpha)}$ is in $\left.\left.\bar{G}^{-1}(] a,+\infty\right]\right)$ for some $\alpha \in \mathbb{R}_{+}$. The function $g^{(\alpha)}+h^{(\alpha)}$ is $2 \alpha$-Lipschitz continuous. Item 2 above shows that $\bar{G}$ coincides with $G$ on Lipschitz continuous maps, so $\bar{G}\left(g^{(\alpha)}+h^{(\alpha)}\right)=G\left(g^{(\alpha)}+h^{(\alpha)}\right)$. Since $G$ is sublinear, the latter is less than or equal to $G\left(g^{(\alpha)}\right)+G\left(h^{(\alpha)}\right)$, so $G\left(g^{(\alpha)}\right)+$ $G\left(h^{(\alpha)}\right)>a$. Since $\bar{G}(g) \geq G\left(g^{(\alpha)}\right)$, and similarly with $h$, we obtain that $\bar{G}(g)+\bar{G}(h)>a$. Since $a$ is arbitrary, $\bar{G}(g)+\bar{G}(h) \geq \bar{G}(g+h)$.

If $G$ is subnormalized, then we show that $\bar{G}$ is subnormalized, too, by a similar argument. Let $\alpha \in \mathbb{R}_{+}, h \in \mathcal{L} X$. Fix $a \in \mathbb{R}_{+}$such that $a<$ $\bar{G}(\alpha . \mathbf{1}+h)$. Then $\alpha . \mathbf{1}+h$ is in the Scott-open set $\left.\left.\bar{G}^{-1}(] a,+\infty\right]\right)$. We observe that $\alpha . \mathbf{1}+h$ is the pointwise supremum of the chain of maps $\alpha . \mathbf{1}+h^{(\beta)}$, $\beta \in \mathbb{R}_{+}$, and that those maps are $\beta$-Lipschitz continuous. Therefore $\alpha . \mathbf{1}+h^{(\beta)}$ is also in $\left.\left.\bar{G}^{-1}(] a,+\infty\right]\right)$, for some $\beta \in \mathbb{R}_{+}$. Since that function is $\beta$-Lipschitz continuous, $\bar{G}$ maps it to $G\left(\alpha .1+h^{(\beta)}\right)$, which is less than or equal to $\alpha+$ $G\left(h^{(\beta)}\right)$ since $G$ is subnormalized. In particular, $a<\bar{G}\left(\alpha \cdot \mathbf{1}+h^{(\beta)}\right) \leq \alpha+G(h)$. Taking suprema over $a$ proves the claim.

If $G$ is normalized, it remains to show that $\bar{G}(\alpha \cdot \mathbf{1}+h) \geq \alpha+G(h)$. This is similar to the argument for the preservation of superlinearity. We use the fact that $\alpha .1+h^{(\beta)} \leq(\alpha .1+h)^{(\beta)}$, which follows from the fact that the left-hand side is $\beta$-Lipschitz continuous below $\alpha . \mathbf{1}+h$. Then $\bar{G}(\alpha . \mathbf{1}+h)=$ $\sup _{\beta \in \mathbb{R}_{+}} G\left((\alpha .1+h)^{(\beta)}\right) \geq \sup _{\beta \in \mathbb{R}_{+}} G\left(\alpha .1+h^{(\beta)}\right)=\sup _{\beta \in \mathbb{R}_{+}}\left(\alpha+G\left(h^{(\beta)}\right)\right)=$ $\alpha+\bar{G}(h)$.

Item 4 is obvious, since by item $2 G$ is the restriction of $\bar{G}$ to $\mathcal{L}_{\infty}(X, d)$.

We can now deduce the following. In the classical situation involving metric spaces, that could be deduced from a simple application of the StoneWeierstrass theorem, a tool that we do not have in the asymmetric situation.

Corollary 3.14. Let $X, d$ be a standard quasi-metric space, and $F, F^{\prime}$ be two previsions on $X$. Then $F=F^{\prime}$ if and only if $F(h)=F^{\prime}(h)$ for every $h \in \mathcal{L}_{1}(X, d)$.

Proof. The only if direction is trivial. In the if direction, for every $\alpha>0$, for every $h \in \mathcal{L}_{\alpha}(X, d), 1 / \alpha h$ is in $\mathcal{L}_{1}(X, d)$. We have $F(1 / \alpha h)=F^{\prime}(1 / \alpha h)$ by assumption. Multiplying by $\alpha$ and relying on positive homogeneity, $F(h)=$ 
$F\left(h^{\prime}\right)$. This shows that $F_{\mid \mathcal{L}_{\infty} X}=F_{\mid \mathcal{L}_{\infty} X}^{\prime}$, whence $F=F^{\prime}$ by Proposition 3.13, item 2 .

Definition $3.15\left(\mathcal{L}_{\infty}^{\mathrm{b}}(X, d)\right)$. Let $\mathcal{L}_{\infty}^{\mathrm{b}}(X, d)$ be the subspace of all bounded maps in $\mathcal{L}_{\infty}(X, d)$, and $\mathcal{L}_{\alpha}^{\mathrm{b}}(X, d)$ be the corresponding subspace of all bounded maps in $\mathcal{L}_{\alpha}(X, d)$, with the subspace topologies.

Lemma 3.16. Let $X, d$ be a quasi-metric space. For every $a>0, \mathcal{L}_{\infty}^{\mathrm{b}}(X, d)=$ $\bigcup_{\alpha>0} \mathcal{L}_{\alpha}^{a}(X, d)$.

Proof. Consider any bounded map $f$ from $\mathcal{L}_{\infty}(X, d)$. By definition, $f \leq b .1$ for some $b \in \mathbb{R}_{+}$, and $f \in \mathcal{L}_{\alpha}(X, d)$ for some $\alpha>0$. Since $\mathcal{L}_{\alpha}(X, d)$ grows as $\alpha$ increases, we may assume that $\alpha \geq b / a$. Then $f$ is in $\mathcal{L}_{\alpha}^{a}(X, d)$. The reverse inclusion is obvious.

Definition 3.17 ( $\mathcal{L}^{\mathrm{b}}$-prevision). Let $X, d$ be a quasi-metric space. An $\mathcal{L}^{\mathrm{b}}$ prevision on $X$ is any continuous map $H$ from $\mathcal{L}_{\infty}^{\mathrm{b}} X$ to $\overline{\mathbb{R}}_{+}$such that $H(\beta h)=$ $\beta H(h)$ for all $\beta \in \mathbb{R}_{+}$and $h \in \mathcal{L}_{\infty}^{\mathrm{b}} X$.

We define linear, superlinear, sublinear, subnormalized, and normalized $\mathcal{L}^{\text {b- }}$ previsions in the usual way.

Every $\mathcal{L}$-prevision $G$ defines a linear $\mathcal{L}^{\mathrm{b}}$-prevision $G_{\mid \mathcal{L}_{\infty}^{\mathrm{b}} X}$. Conversely,

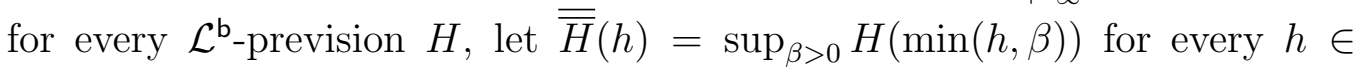
$\mathcal{L}_{\infty}(X, d)$.

Lemma 3.18. Let $X, d$ be a standard quasi-metric space, and $a>0$.

1. For every $H \in \mathcal{L}^{\mathrm{b}} \mathbf{P} X, \overline{\bar{H}}$ is an $\mathcal{L}$-prevision.

2. The maps $G \in \mathcal{L} \mathbf{P} X \mapsto G_{\mid \mathcal{L}_{\infty}^{\mathrm{b}} X} \in \mathcal{L}^{\mathrm{b}} \mathbf{P} X$ and $H \in \mathcal{L}^{\mathrm{b}} \mathbf{P} X \mapsto \overline{\bar{H}} \in \mathcal{L} \mathbf{P} X$ are inverse of each other.

3. For every $G \in \mathcal{L} \mathbf{P} X, G$ is linear, resp. superlinear, resp. sublinear, resp. subnormalized, resp. normalized, if and only if $G_{\mid \mathcal{L}_{\infty}^{\mathrm{b}} X}$ is.

ProOF. We first show that the map $t_{\beta}: \mathcal{L}_{\infty}(X, d) \rightarrow \mathcal{L}_{\infty}^{\mathrm{b}}(X, d)$ defined by $t_{\beta}(h)=\min (h, \beta)$ is continuous for every $\beta>0$. This follows from the definition of subspace topologies, and the fact that $t_{\beta}$ is the restriction of the Scott-continuous map $h \in \mathcal{L} X \mapsto \min (h, \beta) \in \mathcal{L} X$. 
1. $\overline{\bar{H}}$ is continuous, since $\left.\left.(\overline{\bar{H}})^{-1}(] r,+\infty\right]\right)=\left\{h \in \mathcal{L}_{\infty}(X, d) \mid \exists \beta>\right.$ $\left.\left.0, H(\min (h, \beta))>r\}=\bigcup_{\beta>0}\left\{h \in \mathcal{L}_{\infty}(X, d) \mid \min (h, \beta) \in H^{-1}(] r,+\infty\right]\right)\right\}=$ $\left.\left.\bigcup_{\beta>0} t_{\beta}^{-1}\left(H^{-1}(] r,+\infty\right]\right)\right)$, which is open since $H$ and $t_{\beta}$ are continuous.

We must show that $\overline{\bar{H}}(\alpha h)=\alpha \overline{\bar{H}}(h)$ for every $\alpha \in \mathbb{R}_{+}$. When $\alpha>0$, $\overline{\bar{H}}(\alpha h)=\sup _{\beta>0} H(\min (\alpha h, \beta))=\sup _{\beta^{\prime}>0} H\left(\min \left(\alpha h, \alpha \beta^{\prime}\right)\right)=\sup _{\beta^{\prime}>0} \alpha H\left(\min \left(h, \beta^{\prime}\right)\right)$ is equal to $\alpha \overline{\bar{H}}(h)$. When $\alpha=0, \overline{\bar{H}}(0)=\sup _{\beta>0} H(0)=0$.

2. For every $h \in \mathcal{L}_{\infty}^{\mathrm{b}}(X, d),(\overline{\bar{H}})_{\mid \mathcal{L}_{\infty}^{\mathrm{b} X}}(h)=\overline{\bar{H}}(h)=\sup _{\beta>0} H(\min (h, \beta))=$ $H(h)$, since $\min (h, \beta)=h$ for $\beta$ large enough. In the other direction, for every $h \in \mathcal{L}_{\infty}(X, d), \overline{\overline{G_{\mid \mathcal{L}_{\infty}^{\mathrm{b} X} X}}}(h)=\sup _{\beta>0} G_{\mid \mathcal{L}_{\infty}^{\mathrm{b} X} X}(\min (h, \beta))=\sup _{\beta>0} G(\min (h, \beta))$. Recall now from Proposition 3.13 that $G$ is the restriction to $\mathcal{L}_{\infty} X$ of a prevision $F$ on $X$, which is Scott-continuous. So $\overline{\overline{G_{\mid \mathcal{L}_{\infty}^{\mathrm{b}} X}}}(h)=\sup _{\beta>0} F(\min (h, \beta))=$ $F(h)=G(h)$.

3. All the claims follow from the fact that $\min (g, \beta)+\min (h, \gamma) \leq \min (g+$ $h, \beta+\gamma)$ and $\min (g+h, \beta) \leq \min (g, \beta)+\min (h, \beta)$ for all maps $g, h$ and all $\beta, \gamma>0$.

Corollary 3.19. Let $X, d$ be a standard quasi-metric space, and $F, F^{\prime}$ be two previsions on $X$, and $a>0$. Then $F=F^{\prime}$ if and only if $F(h)=F^{\prime}(h)$ for every $h \in \mathcal{L}_{1}^{a}(X, d)$.

Proof. The only if direction is trivial. In the if direction, for every $\alpha>0$, for every $h \in \mathcal{L}_{\alpha}^{a}(X, d), 1 / \alpha h$ is in $\mathcal{L}_{1}^{a}(X, d)$. We have $F(1 / \alpha h)=F^{\prime}(1 / \alpha h)$ by assumption. Multiplying by $\alpha$ and relying on positive homogeneity, $F(h)=F\left(h^{\prime}\right)$. We have $\mathcal{L}_{\infty}^{\mathrm{b}}(X, d)=\bigcup_{\alpha>0} \mathcal{L}_{\alpha}^{a}(X, d)$ [19, Lemma 3.5]. Therefore $F_{\mid \mathcal{L}_{\infty}^{\mathrm{b}} X}=F_{\mid \mathcal{L}_{\infty}^{\mathrm{b}} X}^{\prime}$, whence $F=F^{\prime}$ by Lemma 3.18, item 2, and Proposition 3.13 , item 2 .

\section{Completeness}

\subsection{Basic results}

In the sequel, let $\mathbf{V}_{*} X$ denote $\mathbf{V} X, \mathbf{V}_{\leq 1} X$ or $\mathbf{V}_{1} X$.

Given a standard quasi-metric space $X, d$, we wish to show that, under certain assumptions, $\mathbf{V}_{*} X, d_{\mathrm{KR}}$ is complete. Equivalently, we wish to show that $\mathbf{B}\left(\mathbf{V}_{*} X, d_{\mathrm{KR}}\right)$ is a dcpo. To this end, we consider any directed family $\left(\nu_{i}, r_{i}\right)_{i \in I}$ in $\mathbf{B}\left(\mathbf{V}_{*} X, d_{\mathrm{KR}}\right)$, and we wish to find its least upper bound $(\nu, r)$. If $\mathbf{V}_{*} X, d_{\mathrm{KR}}$ is indeed complete, it is in particular standard, so $r$ must be 
equal to $\inf _{i \in I} r_{i}$. Finding $\nu$ can be done as follows. We form the linear $\mathcal{L}$ prevision $G_{i}$ associated with $\nu_{i}$ for each $i \in I$, namely $G_{i}(h)=\int_{x \in X} h(x) d \nu_{i}$ for every $h \in \mathcal{L}_{\infty}(X, d)$. We look for the linear $\mathcal{L}$-prevision $G$ associated with $\nu$. Inspired by Lemma 3.10, item 2, we bet that $G(h)$ will be equal to $\sup _{i \in I}\left(G_{i}(h)+r-r_{i}\right)$ for every $h \in \mathcal{L}_{1}(X, d)$. We will see that this is a directed supremum. In general, we use the following formula.

Definition 4.1. Let $X, d$ be a standard quasi-metric space, and $*$ be nothing, " $\leq 1$ ", or "1". For every directed family $\left(\nu_{i}, r_{i}\right)_{i \in I}$ in $\mathbf{B}\left(\mathbf{V}_{*} X, d_{K R}\right)$ (resp., $\left.\mathbf{B}\left(\mathbf{V}_{*} X, d_{K R}^{a}\right), a>0\right)$, the pair $\left(G, \inf _{i \in I} r_{i}\right)$, or just the map $G: \mathcal{L}_{\infty}(X, d) \rightarrow$ $\overline{\mathbb{R}}_{+}$(resp., $\left.G: \mathcal{L}_{\infty}^{\mathrm{b}}(X, d) \rightarrow \overline{\mathbb{R}}_{+}\right)$, defined as follows, is called the naive supremum of $\left(\nu_{i}, r_{i}\right)_{i \in I}$ :

$$
G(h)=\sup _{i \in I}\left(\int_{x \in X} h(x) d \nu_{i}+\alpha r-\alpha r_{i}\right)
$$

for every $h \in \mathcal{L}_{\alpha}(X, d)$ (resp., $h \in \mathcal{L}_{\alpha}^{a}(X, d)$ ).

If $G$ is a linear $\mathcal{L}$-prevision (resp., a linear $\mathcal{L}^{\mathrm{b}}$-prevision), then the continuous valuation $\nu$ defined by $\nu(U)=\bar{G}\left(\chi_{U}\right)$ for every $U \in \mathcal{O} X$, will also be called the naive supremum of $\left(\nu_{i}, r_{i}\right)_{i \in I}$.

That generalizes to the case of all previsions, not necessarily linear, as needed in sequels to this work: given any directed family $\left(G_{i}, r_{i}\right)_{i \in I}$ in $\mathbf{B}\left(\mathcal{L} \mathbf{P} X, d_{\mathrm{KR}}\right)$, the naive supremum of the family is the pair $(G, r)$ where $r=\inf _{i \in I} r_{i}$ and, for each $h \in \mathcal{L}_{\alpha}(X, d), \alpha>0, G(h)=\sup _{i \in I}\left(G_{i}(h)+\alpha r-\alpha r_{i}\right)$.

The following shows that the naive supremum is indeed the supremum... provided that it is continuous.

Proposition 4.2. Let $X, d$ be a standard quasi-metric space, and $\left(G_{i}, r_{i}\right)_{i \in I}$ be a directed family in $\mathbf{B}\left(\mathcal{L} \mathbf{P} X, d_{K R}\right)$ (resp, $\left.d_{K R}^{a}, a>0\right)$. Let $r=\inf _{i \in I} r_{i}$ and, for each $h \in \mathcal{L}_{\alpha}(X, d)$ (resp., $\mathcal{L}_{\alpha}^{a}(X, d)$ ), $\alpha>0$, define $G(h)$ as the directed supremum $\sup _{i \in I}\left(G_{i}(h)+\alpha r-\alpha r_{i}\right)$. Then:

1. $G$ is a well-defined, positively homogeneous functional from $\mathcal{L} X$ to $\overline{\mathbb{R}}_{+}$;

2. for any upper bound $\left(G^{\prime}, r^{\prime}\right)$ of $\left(G_{i}, r_{i}\right)_{i \in I}, r^{\prime} \leq r$ and, for every $h \in$ $\mathcal{L}_{\alpha}(X, d)$ (resp., $\mathcal{L}_{\alpha}^{a}(X, d)$ ) with $\alpha>0, G(h) \leq G^{\prime}(h)+\alpha r-\alpha r^{\prime} ;$

3. if $G$ is continuous, then $(G, r)$ is the supremum of $\left(G_{i}, r_{i}\right)_{i \in I}$; 
4. if every $G_{i}$ is sublinear, resp. superlinear, resp. linear, resp. subnormalized, resp. normalized, then so is $G$.

ProOF. We only deal with the $d_{\mathrm{KR}}$ case, since the $d_{\mathrm{KR}}^{a}$ case is similar.

We check that $\sup _{i \in I}\left(G_{i}(h)+\alpha r-\alpha r_{i}\right)$ is a directed supremum, for every $h \in \mathcal{L}_{\alpha}(X, d)$. Define $\sqsubseteq$ by $i \sqsubseteq j$ if and only if $\left(G_{i}, r_{i}\right) \leq d_{\mathrm{KR}}^{+}\left(G_{j}, r_{j}\right)$. Then $\sqsubseteq$ turns $I$ into a directed preordered set, and the family $\left(G_{i}, r_{i}\right)_{i \in I}$

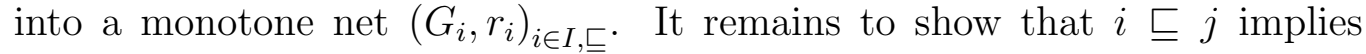
$G_{i}(h)+\alpha r-\alpha r_{i} \leq G_{j}(h)+\alpha r-\alpha r_{j}$. Since $\left(G_{i}, r_{i}\right) \leq d_{\mathrm{KR}}^{+}\left(G_{j}, r_{j}\right)$, and $1 / \alpha h$ is in $\mathcal{L}_{1}(X, d), G_{i}(1 / \alpha h) \leq G_{j}(1 / \alpha h)+r_{i}-r_{j}$. We obtain the result by multiplying both sides by $\alpha$ and adding $\alpha r$.

1. We must first show that $G$ is well-defined, in the following sense. When $h \in \mathcal{L}_{\alpha} X, h$ is also in $\mathcal{L}_{\beta} X$ for every $\beta \geq \alpha$, and our tentative definition is not

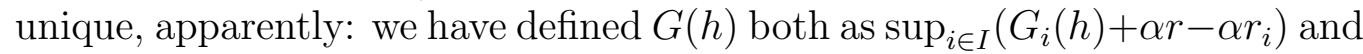

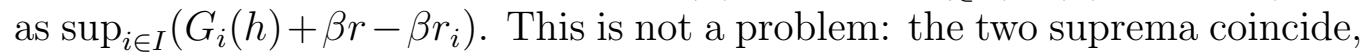
since $G_{i}(h)+\alpha r-\alpha r_{i}$ and $G_{i}(h)+\beta r-\beta r_{i}$ only differ by $(\beta-\alpha)\left(r_{i}-r\right)$, which can be made arbitrarily small as $i$ varies in $I$. In fact, both "definitions" are equal to $\lim _{i \in I, \sqsubseteq} G_{i}(h)$, where the limit is taken in $\overline{\mathbb{R}}_{+}$with its usual Hausdorff topology, a basis of which is given by the intervals $[0, b[] a,, b[$ and ] $a,+\infty], 0<a<b<+\infty$. This remark will be helpful in the sequel. In particular, taking the definition $G(h)=\lim _{i \in I, \sqsubseteq} G_{i}(h)$, it is easy to show that $G$ commutes with products with non-negative constants, which finishes to prove item 1 .

We proceed with item 4 , and we will return to items 2 and 3 later.

4. Using again the formula $G(h)=\lim _{i \in I, \sqsubseteq} G_{i}(h)$, if every $G_{i}$ is sublinear, resp. superlinear, resp. linear, resp. subnormalized, resp. normalized, then so is $G$, because because + and products by scalars are continuous on $\overline{\mathbb{R}}_{+}$with its usual Hausdorff topology.

2. Let $\left(G^{\prime}, r^{\prime}\right)$ be any upper bound of $\left(G_{i}, r_{i}\right)_{i \in I}$. For every $i \in I$, for every $h \in \mathcal{L}_{1}(X, d)$ the inequality $\left(G_{i}, r_{i}\right) \leq_{\mathrm{KR}}^{+}\left(G^{\prime}, r^{\prime}\right)$, equivalently $d_{\mathrm{KR}}\left(G_{i}, G^{\prime}\right) \leq$ $r_{i}-r^{\prime}$, implies that $G_{i}(h) \leq G^{\prime}(h)+r_{i}-r^{\prime}$ (and $r_{i} \geq r^{\prime}$ ). Then $G_{i}(h)-$ $r_{i} \leq G^{\prime}(h)-r^{\prime}$. Taking suprema over $i \in I, G(h)-r \leq G^{\prime}(h)-r^{\prime}$, hence $G(h) \leq G^{\prime}(h)+r-r^{\prime}$ (and, by taking infima, $r=\inf _{i \in I} r_{i} \geq r^{\prime}$ ). Now take any $h \in \mathcal{L}_{\alpha}(X, d), \alpha>0$. Then $1 / \alpha h$ is in $\mathcal{L}_{1}(X, d)$, and by positive homogeneity $G(h) \leq G^{\prime}(h)+\alpha r-\alpha r^{\prime}$.

3. Assume $G$ continuous. The definition of $G$ ensures that $(G, r)$ is an element of $\mathbf{B}\left(\mathcal{L} \mathbf{P} X, d_{\mathrm{KR}}\right)$, and is an upper bound of $\left(G_{i}, r_{i}\right)_{i \in I}$. For every 
upper bound $\left(G^{\prime}, r^{\prime}\right)$ of $\left(G_{i}, r_{i}\right)_{i \in I}$, item 2 entails that $r \geq r^{\prime}$ and, for every $h \in \mathcal{L}_{1}(X, d), G(h) \leq G^{\prime}(h)+r-r^{\prime}$, whence $d_{\mathrm{KR}}\left(G, G^{\prime}\right) \leq r-r^{\prime}$. It follows that $(G, r) \leq d_{\mathrm{KR}}^{+}\left(G^{\prime}, r^{\prime}\right)$, showing that $(G, r)$ is the least upper bound.

The following is not quite the completeness result we are after, because of the awkward assumption that the topology of $\mathcal{L}_{\infty}(X, d)$ is determined by those of $\mathcal{L}_{\alpha}(X, d), \alpha>0$, or equivalently that: any subset $U$ of $\mathcal{L}_{\infty}(X, d)$ such that $U \cap \mathcal{L}_{\alpha}(X, d)$ is open in $\mathcal{L}_{\alpha}(X, d)$ for every $\alpha>0$ is open in $\mathcal{L}_{\infty}(X, d)$, or also that: every map $G: \mathcal{L}_{\infty}(X, d) \rightarrow Z$, where $Z$ is any topological space, whose restriction to $\mathcal{L}_{\alpha}(X, d)$ is continuous for every $\alpha>0$, is itself continuous.

Theorem 4.3. Let $X, d$ be a standard quasi-metric space, and assume that the topology of $\mathcal{L}_{\infty}(X, d)$ is determined by those of $\mathcal{L}_{\alpha}(X, d), \alpha>0$ (resp., the topology of $\mathcal{L}_{\infty}^{\mathrm{b}}(X, d)$ is determined by those of $\mathcal{L}_{\alpha}^{a}(X, d), \alpha>0$, where $a>0$ is fixed).

Then $\mathbf{V} X, \mathbf{V}_{\leq 1} X$ and $\mathbf{V}_{1} X$ with the $d_{K R}$ quasi-metric (resp., $d_{K R}^{a}, a>0$ ) are complete, and all suprema of directed families of formal balls of continuous valuations are naive suprema.

Proof. We only deal with the case of $d_{\mathrm{KR}}$, as the $d_{\mathrm{KR}}^{a}$ case is similar. We take the notations of Definition 4.1. Considering Proposition 4.2, item 3, it suffices to show that the restriction $G_{\mid \mathcal{L}_{\alpha} X}$ of $G$ is continuous from $\mathcal{L}_{\alpha}(X, d)$ to $\overline{\mathbb{R}}_{+}$, for any $\alpha>0$. Fix $a \in \mathbb{R}$. We must show that $\mathcal{U}=\left\{h \in \mathcal{L}_{\alpha}(X, d) \mid\right.$ $G(h)>a\}$ is open in $\mathcal{L}_{\alpha}(X, d)$. Using the definition of $G$, we write $\mathcal{U}$ as the set of maps $h \in \mathcal{L}_{\alpha}(X, d)$ such that $\int_{x \in X} h(x) d \nu_{i}+\alpha r-\alpha r_{i}>a$ for some $i \in I$. Therefore $\mathcal{U}=\bigcup_{i \in I}\left[h>a+\alpha r_{i}-\alpha r,+\infty\right] \cap \mathcal{L}_{\alpha}(X, d)$, which is open.

Remark 4.4. Proposition 8.2 of [19] states that, if $X, d$ is a Lipschitz regular, standard quasi-metric space, then the topology of $\mathcal{L}_{\infty}(X, d)$ is determined by those of $\mathcal{L}_{\alpha}(X, d), \alpha>0$ (resp., Proposition 8.3 states the same thing for $\mathcal{L}_{\infty}^{\mathrm{b}}(X, d)$ and $\left.\mathcal{L}_{\alpha}^{a}(X, d), \alpha>0\right)$. Hence Theorem 4.3 in particular applies to all Lipschitz regular standard quasi-metric spaces. Those include all algebraic complete quasi-metric with relatively compact balls, see [18, Corollary 4.7]. They also include spaces of formal balls $\mathbf{B}(Y, \partial), \partial^{+}$, for every quasi-metric space $Y, \partial[18$, Theorem 4.13].

The strategy we will use to obtain our next completeness result uses the latter observation as follows. Recall that there is a map $\eta_{X}: X \rightarrow \mathbf{B}(X, d)$ defined 
by $\eta_{X}(x)=(x, 0)$. We start from a directed family $\left(\nu_{i}, r_{i}\right)_{i \in I}$ in $\mathbf{B}\left(\mathbf{V}_{*} X, d_{\mathrm{KR}}\right)$ again, and we consider the family $\left(\eta_{X}\left[\nu_{i}\right], r_{i}\right)_{i \in I}$ in $\mathbf{B}\left(\mathbf{V}_{*}(\mathbf{B}(X, d)), d^{+}{ }_{\mathrm{KR}}\right)$. That will happen to be directed again. Since $\mathbf{B}(X, d)$ is Lipschitz regular, $\left(\eta_{X}\left[\nu_{i}\right], r_{i}\right)_{i \in I}$ has a (naive) supremum $(\mu, r)$ by Remark 4.4. We will show that $\mu$ is supported on $X$, so that $\mu=\eta_{X}[\nu]$ for some $\nu \in \mathbf{V} X$, and we will check that $\nu$ is then the (naive) supremum of the original family $\left(\nu_{i}, r_{i}\right)_{i \in I}$. This will work as soon as $X, d$ is a continuous complete quasi-metric space.

We must have a look at supports first.

\subsection{Supports}

Definition 4.5 (Support). Let $X$ be a topological space. A subset $A$ of $X$ is called a support of a continuous valuation $\nu$ if and only if for all open subsets $U$ and $V$ of $X$ such that $U \cap A=V \cap A, \nu(U)=\nu(V)$. We also say that $\nu$ is supported on $A$ in that case.

Lemma 4.6. Let $X$ be a topological space, $A$ be a subset of $X$, and $\mu$ be a continuous valuation on $X$. Then $\mu$ is supported on $A$ if and only if there is continuous valuation $\nu$ on $A$ such that $\mu=i[\nu]$, where $i$ is the inclusion map from $A$ into $X$.

Proof. If $\mu=i[\nu]$, then $\mu(U)=\nu\left(i^{-1}(U)\right)=\nu(U \cap A)$, so $\mu$ is clearly supported on $A$. Conversely, let us assume that $\mu$ is supported on $A$, and let us define $\nu(U)$, for every $U \in \mathcal{O} A$, as $\mu(V)$, where $V$ is any open subset of $X$ such that $V \cap A=U$. Which $V$ it is is unimportant, by the definition of supports. It is easy to see that $\nu$ is strict and modular. For monotonicity, we must show that $V_{1} \cap A \subseteq V_{2} \cap A$ implies $\mu\left(V_{1}\right) \leq \mu\left(V_{2}\right)$. This follows from the fact that $V_{2} \cap A=\left(V_{1} \cup V_{2}\right) \cap A$ : we have $\mu\left(V_{1}\right) \leq \mu\left(V_{1} \cup V_{2}\right)=$ $\mu\left(V_{2}\right)$. For Scott-continuity, let $\left(V_{i} \cap A\right)_{i \in I}$ be a directed family in $\mathcal{O} A$, where each $V_{i}$ is open in $X$. The inequality $\sup _{i \in I} \mu\left(V_{i}\right) \leq \mu\left(\bigcup_{i \in I} V_{i}\right)$ follows from monotonicity. In the converse direction, let $V_{i}^{\prime}=\bigcup\left\{V_{j} \mid j \in I, V_{j} \cap A \subseteq\right.$ $\left.V_{i} \cap A\right\}$. Then $\left(V_{i}^{\prime}\right)_{i \in I}$ is directed, so $\sup _{i \in I} \mu\left(V_{i}^{\prime}\right)=\mu\left(\bigcup_{i \in I} V_{i}^{\prime}\right)$. However, $V_{i}^{\prime} \cap A=V_{i} \cap A$, so $\mu\left(V_{i}^{\prime}\right)=\mu\left(V_{i}\right)$ for every $i \in I$. Similarly, $\left(\bigcup_{i \in I} V_{i}^{\prime}\right) \cap A=$ $\bigcup_{i \in I}\left(V_{i}^{\prime} \cap A\right)=\bigcup_{i \in I}\left(V_{i} \cap A\right)=\left(\bigcup_{i \in I} V_{i}\right) \cap A$, so $\mu\left(\bigcup_{i \in I} V_{i}^{\prime}\right)=\mu\left(\bigcup_{i \in I} V_{i}\right)$, whence $\mu\left(\bigcup_{i \in I} V_{i}\right)=\sup _{i \in I} \mu\left(V_{i}\right)$.

Remark 4.7. If $A$ is open in $X$, then $A$ is a support of $\nu$ if and only if, for every open subset $U$ of $X, \nu(U)=\nu(U \cap A)$. This definition should appear more familiar. 


\subsection{Push-forwards}

Lemma 4.8. Let $*$ be nothing, " $\leq 1$ ", or " $1 "$. Let $X, d$ and $Y, \partial$ be two quasi-metric spaces, and $f: X, d \mapsto Y, \partial$ be an $\alpha$-Lipschitz continuous map, where $\alpha>0$. Then $\mathbf{V} f: \mathbf{V}_{*} X, d_{K R} \rightarrow \mathbf{V}_{*} Y, \partial_{K R}$, defined by:

$$
\mathbf{V} f(\nu)=f[\nu]
$$

is an $\alpha$-Lipschitz map. A similar result holds for $\mathbf{V} f$, seen as a map from $\mathbf{V} X, d_{K R}^{a}$ to $\mathbf{V} Y, \partial_{K R}^{a}$, for every $a \in \mathbb{R}_{+}, a>0$.

Proof. Let $\nu, \nu^{\prime}$ be two continuous valuations on $X$. For every $h \in \mathcal{L}_{1}(Y, \partial)$, $\int_{y \in Y} h(y) d f[\nu]=\int_{x \in X} h(f(x)) d \nu$ by the change of variable formula, and similarly with $\nu^{\prime}$. Observing that $h \circ f$ ranges over a subset of the set of all $\alpha$-Lipschitz continuous maps, equivalently of maps of the form $\alpha k$ with $k \in$ $\mathcal{L}_{1}(X, d), \partial_{\mathrm{KR}}\left(f[\nu], f\left[\nu^{\prime}\right]\right) \leq \sup _{k \in \mathcal{L}_{1}(X, d)} \mathrm{d}_{\mathbb{R}}\left(\int_{x \in X} \alpha k(x) d \nu, \int_{x \in X} \alpha k(x) d \nu^{\prime}\right)=$ $\alpha d_{\mathrm{KR}}\left(\nu, \nu^{\prime}\right)$. The case of $d_{\mathrm{KR}}^{a}$ and $\partial_{\mathrm{KR}}^{a}$ is similar, noting that if $h$ takes its values in $[0, \alpha a]$, then so does $h \circ f$.

We cannot prove that $\mathbf{V} f$ is $\alpha$-Lipschitz continuous (yet), but we have the following.

Lemma 4.9. Let $X, d$ and $Y, \partial$ be two quasi-metric spaces, and $f: X, d \mapsto$ $Y, \partial$ be a 1-Lipschitz continuous map. Let also * be nothing, " $\leq 1$ ", or "1". For every directed family of formal balls $\left(\nu_{i}, r_{i}\right), i \in I$, in $\mathbf{B}\left(\mathbf{V}_{*} X, d_{K R}\right)$ (resp., $\left.d_{K R}^{a}\right)$, with naive supremum $(\nu, r),(\mathbf{V} f(\nu), r)$ is the naive supremum of $\left(\mathbf{V} f\left(\nu_{i}\right), r_{i}\right)_{i \in I}$.

Proof. More generally, we show that if $G$ is the naive supremum of $\left(\nu_{i}, r_{i}\right)_{i \in I}$, in the sense of Definition 4.1, then the map $G^{\prime}$ defined by $G^{\prime}(k)=G(k \circ f)$ is the naive supremum of $\left(\mathbf{V} f\left(\nu_{i}\right), r_{i}\right)_{i \in I}$. The claim will follow in case $G$ is a linear $\mathcal{L}$-prevision, taking its associated continuous valuation $\nu$, in which case $G^{\prime}(k)=\int_{x \in X} k(f(x)) d \nu=\int_{y \in Y} k(y) d f[\nu]$, so that $f[\nu]=\mathbf{V} f(\nu)$ is the continuous valuations associated with $G^{\prime}$.

By definition, $r=\inf _{i \in I} r_{i}$, and $G(h)=\sup _{i \in I}\left(\int_{x \in X} h(x) d \nu_{i}+\alpha r-\alpha r_{i}\right)$ for every $h \in \mathcal{L}_{\alpha}(X, d)$ (resp., in $\mathcal{L}_{\alpha}^{a}(X, d)$ ), for every $\alpha \in \mathbb{R}_{+}, \alpha>0$. It follows that, for every $k \in \mathcal{L}_{\alpha}(Y, \partial)$ (resp., in $\mathcal{L}_{\alpha}^{a}(Y, \partial)$ ), and using the fact that $k \circ f$ is in $\mathcal{L}_{\alpha}(X, d)$ (resp., in $\mathcal{L}_{\alpha}^{a}(X, d)$, since $k \circ f$ is bounded by $\alpha a$, just like $k), G^{\prime}(k)=G(k \circ f)=\sup _{i \in I}\left(\int_{x \in X} k\left(f(x) d \nu_{i}\right)+\alpha r-\alpha r_{i}\right)=$ $\sup _{i \in I}\left(\int_{y \in Y} k(y) d \mathbf{V} f\left(\nu_{i}\right)+\alpha r-\alpha r_{i}\right)$. 


\subsection{Working on the space of formal balls}

We now use the following results on formal balls. Theorem 3.14 of [18] states that there is a monad $(\mathbf{B}, \eta, \nu)$ on the category of standard quasimetric spaces and 1-Lipschitz continuous maps, where $\eta_{X}: X \rightarrow \mathbf{B}(X, d)$ is defined by $\eta_{X}(x)=(x, 0)$, and $\mu_{X}: \mathbf{B}\left(\mathbf{B}(X, d), d^{+}\right) \rightarrow \mathbf{B}(X, d)$ is defined by $\mu_{X}\left((x, r), r^{\prime}\right)=\left(x, r+r^{\prime}\right)$. Also, the action of $\mathbf{B}$ on morphisms is given by $\mathbf{B}(f)=\mathbf{B}^{1}(f)$, namely $\mathbf{B}(f)(x, r)=(f(x), r)$. Note in particular that $\eta_{X}$ is 1-Lipschitz continuous (that is Lemma 3.8 of the same paper), and that $\mathbf{B}(X, d), d^{+}$is standard for every standard space $X, d$ (Proposition 3.11).

For every continuous valuation $\nu$ on $X, \mathbf{V} \eta_{X}(\nu)$ is a continuous valuation on $\mathbf{B}(X, d)$, which one can see as an extension of $\nu$ from $X$ to its superspace $\mathbf{B}(X, d)$. Moreover, by Lemma 4.8, $\mathbf{V} \eta_{X}$ is 1-Lipschitz, so $\mathbf{B}\left(\mathbf{V} \eta_{X}\right)$ is monotonic. This implies that for every directed family $\left(\nu_{i}, r_{i}\right)_{i \in I}$ in $\mathbf{B}\left(\mathbf{V}_{*} X, d_{\mathrm{KR}}\right)$ (resp, $\left.\mathbf{B}\left(\mathbf{V}_{*} X, d_{\mathrm{KR}}^{a}\right), a>0\right),\left(\mathbf{V} \eta_{X}\left(\nu_{i}\right), r_{i}\right)_{i \in I}$ is also a directed family, this time in $\mathbf{B}\left(\mathbf{V}_{*}(\mathbf{B}(X, d)), d^{+}{ }_{\mathrm{KR}}\right)\left(\right.$ resp, $\left.d_{\mathrm{KR}}^{+a}\right)$. By Remark 4.4, the latter space is Lipschitz regular, so $\left(\mathbf{V} \eta_{X}\left(\nu_{i}\right), r_{i}\right)_{i \in I}$ has a supremum $(\mu, r)$, which coincides with the naive supremum. In other words, $r=\inf _{i \in I} r_{i}$, and:

$$
\int_{(x, r) \in \mathbf{B}(X, d)} k(x, r) d \mu=\sup _{i \in I}\left(\int_{x \in X} k\left(\eta_{X}(x)\right) d \nu_{i}+\alpha r-\alpha r_{i}\right)
$$

for every $k \in \mathcal{L}_{\alpha}\left(\mathbf{B}(X, d), d^{+}\right), \alpha>0$ (resp., $\left.k \in \mathcal{L}_{\alpha}^{a}\left(\mathbf{B}(X, d), d^{+}\right)\right)$.

(7) seems to imply that $\mu$ is supported on $X$, because $\int_{(x, r) \in \mathbf{B}(X, d)} k(x, r) d \mu$ only depends on $k$ through $k \circ \eta_{X}$. This is deceiving: (7) is only about $\alpha$ Lipschitz continuous maps $k$, not about all lower semicontinuous maps, and indeed (7) only characterizes the linear $\mathcal{L}$-prevision associated with $\mu$.

If we were able to assert that $\mu$ is supported on $X$, then Lemma 4.6 would tell us that $\mu=\eta_{X}[\nu]$ for some continuous valuation $\nu$ on $X$, and then we would be on a good track to show that $(\nu, r)$ is the naive supremum of $\left(\nu_{i}, r_{i}\right)_{i \in I}$. However, for now, the best we will be able to prove is that $\mu$ is supported on the arbitrary small open neighborhoods $V_{\epsilon}$ of $X$ in $\mathbf{B}(X, d)$. Recall that $V_{\epsilon}$ is the set of formal balls of radius strictly less than $\epsilon$. We will do this with the help of the following lemma, which is a fundamental property of the largest $\alpha$-Lipschitz continuous map $\chi_{V}^{(\alpha)}$ below $\chi_{V}$.

Lemma 4.10. Let $X, d$ be a standard quasi-metric space, $V$ be an open subset of $\mathbf{B}(X, d)$ and $\alpha \in \mathbb{R}_{+}, \epsilon>0$. For every $x \in X, \chi_{V}^{(\alpha)}(x, 0)=$ $\min \left(1, \alpha \sup \left\{r^{\prime} \in \mathbb{R}_{+} \mid\left(x, r^{\prime}\right) \in V\right\}\right)$. 
ProOF. We take some help from Proposition 6.14 of [20], which gives an explicit form of $\chi_{V}^{(\alpha)}$, where $V$ is an open subset of a standard quasi-metric space. Specializing it to the standard quasi-metric space $\mathbf{B}(X, d), d^{+}$, we obtain: $\chi_{V}^{(\alpha)}(x, r)=\min \left(1, \alpha d^{+}((x, r), \bar{V})\right)$, where $d^{+}((x, r), \bar{V})=\sup \left\{r^{\prime} \in \mathbb{R}_{+}\right.$ $\left.\left((x, r), r^{\prime}\right) \in \widehat{V}\right\}$, and $\widehat{V}$ is the largest Scott-open subset of $\mathbf{B}\left(\mathbf{B}(X, d), d^{+}\right)$ such that $\eta_{\mathbf{B}(X, d)}^{-1}(\widehat{V})=V$. Theorem 4.13 of [18] states that $\widehat{V}=\mu_{X}^{-1}(V)$, where $\mu_{X}$ is the multiplication of the $\mathbf{B}$ monad. Hence $d^{+}((x, r), \bar{V})=$ $\sup \left\{r^{\prime} \in \mathbb{R}_{+} \mid \mu_{X}\left((x, r), r^{\prime}\right) \in V\right\}=\sup \left\{r^{\prime} \in \mathbb{R}_{+} \mid\left(x, r+r^{\prime}\right) \in V\right\}$. In particular, $\chi_{V}^{(\alpha)}(x, 0)=\min \left(1, \alpha \sup \left\{r^{\prime} \in \mathbb{R}_{+} \mid\left(x, r^{\prime}\right) \in V\right\}\right)$.

Corollary 4.11. Let $X, d$ be a standard quasi-metric space, and $\alpha \in \mathbb{R}_{+}$, $\epsilon>0$. If $\alpha \epsilon \geq 1$, then $\chi_{V}^{(\alpha)} \circ \eta_{X}=\chi_{V \cap V_{\epsilon}}^{(\alpha)} \circ \eta_{X}$.

Proof. By Lemma 4.10, $\left(\chi_{V}^{(\alpha)} \circ \eta_{X}\right)(x)=\chi_{V}^{(\alpha)}(x, 0)=\min \left(1, \alpha \sup \left\{r^{\prime} \in \mathbb{R}_{+} \mid\right.\right.$ $\left.\left.\left(x, r^{\prime}\right) \in V\right\}\right)$. The latter sup is unchanged if we bound the allowed values of $r^{\prime}$ from above by $\epsilon$ (strictly), since all the other values will contribute at least $\alpha \epsilon \geq 1$ to the min. Therefore $\left(\chi_{V}^{(\alpha)} \circ \eta_{X}\right)(x)$ is equal to $\min \left(1, \alpha \sup \left\{r^{\prime} \in\right.\right.$ $\left[0, \epsilon\left[\mid\left(x, r^{\prime}\right) \in V\right\}\right)=\min \left(1, \alpha \sup \left\{r^{\prime} \in \mathbb{R}_{+} \mid\left(x, r^{\prime}\right) \in V \cap V_{\epsilon}\right\}\right)$, which is equal to $\left(\chi_{V \cap V_{\epsilon}}^{(f \alpha)} \circ \eta_{X}\right)(x)$.

Lemma 4.12. Let $X, d$ be a standard quasi-metric space. Let $G$ be a linear $\mathcal{L}$-prevision on $\mathbf{B}(X, d)$ such that, for every $\alpha>0$, for all $f, g \in \mathcal{L}_{\alpha}\left(\mathbf{B}(X, d), d^{+}\right)$ such that $f \circ \eta_{X}=g \circ \eta_{X}, G(f)=G(g)$. Then, for every $\epsilon>0$, the continuous valuation $\mu$ associated with the linear prevision $\bar{G}$ is supported on $V_{\epsilon}$.

Similarly, let $H$ be a linear $\mathcal{L}^{\mathrm{b}}$-prevision on $\mathbf{B}(X, d)$ such that, for every $\alpha>0$, for all $f, g \in \mathcal{L}_{\alpha}^{a}\left(\mathbf{B}(X, d), d^{+}\right)$such that $f \circ \eta_{X}=g \circ \eta_{X}, H(f)=H(g)$. Then, for every $\epsilon>0$, the continuous valuation $\mu$ associated with the linear prevision $\bar{G}$ (where $G=\overline{\bar{H}}$ ) is supported on $V_{\epsilon}$.

Proof. By definition, for every open subset $V$ of $\mathbf{B}(X, d), \mu(V)=\bar{G}\left(\chi_{V}\right)=$ $\sup _{\alpha \in \mathbb{R}_{+}} G\left(\chi_{V}^{(\alpha)}\right)$. When $\alpha$ is large enough, $\chi_{V}^{(\alpha)} \circ \eta_{X}=\chi_{V \cap V_{\epsilon}}^{(\alpha)} \circ \eta_{X}$ by Corollary 4.11, so $G\left(\chi_{V}^{(\alpha)}\right)=G\left(\chi_{V \cap V_{\epsilon}}^{(\alpha)}\right)$. It follows that $\mu(V)$ is also equal to $\sup _{\alpha \in \mathbb{R}_{+}} G\left(\chi_{V \cap V_{\epsilon}}^{(\alpha)}\right)=\bar{G}\left(\chi_{V \cap V_{\epsilon}}\right)=\mu\left(V \cap V_{\epsilon}\right)$.

Given any two open subsets $U$ and $V$ of $\mathbf{B}(X, d)$ such that $U \cap V_{\epsilon}=V \cap V_{\epsilon}$, it follows that $\mu(U)=\mu\left(U \cap V_{\epsilon}\right)=\mu\left(V \cap V_{\epsilon}\right)=\mu(V)$.

The second part of the lemma is proved in exactly the same way, making sure to take $\alpha$ large enough so that $a \alpha \geq 1$, hence that $\chi_{V}^{(\alpha)}$ is in $\mathcal{L}_{\alpha}^{a}(X, d)$. 
Lemma 4.13. Let $*$ be nothing, " $\leq 1$ ", or "1". Let $X, d$ be a standard quasi-metric space, $\left(\nu_{i}, r_{i}\right)_{i \in I}$ be a directed family in $\mathbf{B}\left(\mathbf{V}_{*} X, d_{K R}\right)$ (resp., $\left.\mathbf{B}\left(\mathbf{V}_{*} X, d_{K R}^{a}\right)\right)$, let $r=\inf _{i \in I} r_{i}$ and let $\mu$ be the unique continuous valuation on $\mathbf{B}(X, d)$ satisfying (7). For every $\epsilon>0, \mu$ is supported on $V_{\epsilon}$.

Proof. In the case of $d_{\mathrm{KR}}$, Equation $(7)$ states that the linear $\mathcal{L}$-prevision $G$ associated with $\mu$ (i.e., the left-hand side is just $G(k)$ ) satisfies the assumptions of Lemma 4.12, since the right-hand side only depends on $k$ through $k \circ \eta_{X}$. Hence $\mu$ is supported on $V_{\epsilon}$. With $d_{\mathrm{KR}}^{a}, G$ is a linear $\mathcal{L}^{\mathrm{b}}$-prevision instead, and we use the second part of Lemma 4.12 instead of the first one.

It is rather frustrating to know that $\mu$ is supported on every $V_{\epsilon}$, but not on $X$, which is their intersection. This is solved easily by switching to measures.

\subsection{Extending to measures}

Being supported on every $V_{\epsilon}$ does not imply being supported on their intersection, $X$, or at least I do not know a proof of that without any further assumption on $X$. The implication holds if $X, d$ is continuous complete: this will allow us to use a measure extension theorem and conclude. The key is the following lemma. Recall from Remark 4.7 that a continuous valuation $\mu$ is supported on an open set $W$ if and only if $\mu(U)=\mu(U \cap W)$ for every open set $U$.

Lemma 4.14. Let $Z$ be a space on which every bounded continuous valuation extends to a measure on the Borel $\sigma$-algebra. Let $W_{0} \supseteq W_{1} \supseteq \cdots \supseteq W_{n} \supseteq \cdots$ be a countable descending sequence of open subsets of $Z$, and $X=\bigcap_{n \in \mathbb{N}} W_{n}$. Every bounded continuous valuation $\mu$ on $Z$ that is supported on every $W_{n}$ is supported on $X$.

Proof. Let us write $\mu$ again for an extension of $\mu$ as a (necessarily bounded) measure on $Z$. For every open subset $W$ of $Z$, we have:

$$
\begin{array}{rlr}
\mu(W \cap X) & =\mu\left(\bigcap_{n \in \mathbb{N}} W \cap W_{n}\right) & \\
& =\inf _{n \in \mathbb{N}} \mu\left(W \cap W_{n}\right) & \text { valid for all bounded measures } \\
& =\inf _{n \in \mathbb{N}} \mu(W) & \mu \text { is supported on } W_{n} \\
& =\mu(W) .
\end{array}
$$


Theorem 4.15. Let $X, d$ be a continuous complete quasi-metric space. The spaces $\mathbf{V}_{\leq 1} X$ and $\mathbf{V}_{1} X$, equipped with the $d_{K R}$, resp. the $d_{K R}^{a}$ quasi-metric $(a>0)$, are complete.

Moreover, directed suprema $(\nu, r)$ of formal balls $\left(\nu_{i}, r_{i}\right)_{i \in I}$ are computed as naive suprema: $r=\inf _{i \in I} r_{i}$ and for every $h \in \mathcal{L}_{\alpha}(X, d)$ (resp., in $\left.\mathcal{L}_{\alpha}^{a}(X, d)\right)$,

$$
\int_{x \in X} h(x) d \nu=\sup _{i \in I}\left(\int_{x \in X} h(x) d \nu_{i}+\alpha r-\alpha r_{i}\right) .
$$

Proof. When $X, d$ is continuous complete, $\mathbf{B}(X, d)$ is a continuous dcpo, in particular it is LCS-complete, so every continuous valuation extends to a measure on the Borel $\sigma$-algebra. Hence, given any directed family $\left(\nu_{i}, r_{i}\right)_{i \in I}$ in $\mathbf{B}\left(\mathbf{V}_{*} X, d_{\mathrm{KR}}\right)$ (resp., in $\mathbf{B}\left(\mathbf{V}_{*} X, d_{\mathrm{KR}}^{a}\right), a>0$ ), where each $\nu_{i}$ is a subprobability valuation on $X$, let $r=\inf _{i \in I} r_{i}$ and let $\mu$ be the unique continuous valuation on $\mathbf{B}(X, d)$ satisfying (7). That $\mu$ is again a subprobability valuation (take $k=\mathbf{1}$ ), and it is supported on every $V_{1 / 2^{n}}, n \in \mathbb{N}$, by Lemma 4.13. By Lemma 4.14, $\mu$ is supported on $X=\bigcap_{n \in \mathbb{N}} V_{1 / 2^{n}}$. Hence, by Lemma 4.6, $\mu=\eta_{X}[\nu]$ for some unique continuous valuation $\nu$ on $X$.

We use (7) on certain specific maps $k \in \mathcal{L}_{\alpha}\left(\mathbf{B}(X, d), d^{+}\right)$(resp., $k \in$ $\left.\mathcal{L}_{\alpha}^{a}\left(\mathbf{B}(X, d), d^{+}\right)\right)$. In order to build them, we first note that the function $\partial\left({ }_{-}, y^{\prime}\right): y \mapsto \partial\left(y, y^{\prime}\right)$ is a 1-Lipschitz continuous map from $Y, \partial$ to $\overline{\mathbb{R}}_{+}, \mathbf{d}_{\mathbb{R}}$, for every standard quasi-metric space $Y, \partial[18$, Lemma 2.6$]$. Taking $\mathbf{B}\left(\overline{\mathbb{R}}_{+}, \mathrm{d}_{\mathbb{R}}\right), \mathrm{d}_{\mathbb{R}}^{+}$ for $Y, \partial$ (which is standard, since $\overline{\mathbb{R}}_{+}, \mathrm{d}_{\mathbb{R}}$ is complete hence standard), and $y^{\prime}=$ $(0,0)$, we obtain that the map $m:(x, r) \mapsto \mathrm{d}_{\mathbb{R}}^{+}((x, r),(0,0))=\max (x-r, 0)$ is 1-Lipschitz continuous. For every $h \in \mathcal{L}_{\alpha}(X, d)$ (resp., $\left.\mathcal{L}_{\alpha}^{a}(X, d)\right), 1 / \alpha h$ is 1-Lipschitz continuous, so $\mathbf{B}^{1}(1 / \alpha h)$ is also 1-Lipschitz continuous (recall that $\mathbf{B}$ is the functor part of a monad, hence maps morphisms to morphisms), and therefore $k=\alpha .\left(m \circ \mathbf{B}^{1}(1 / \alpha h)\right)$ is $\alpha$-Lipschitz continuous. Explicitly, $k(x, r)=\alpha m(1 / \alpha h(x), r)=\alpha \max (1 / \alpha h(x)-r, 0)=\max (h(x)-\alpha r, 0)$.

Note that $k \circ \eta_{X}=h$. Plugging that $k$, and $\eta_{X}[\nu]$ for $\mu$, in (7), we obtain that $\int_{(x, r) \in \mathbf{B}(X, d)} \max (h(x)-\alpha r, 0) d \eta_{X}[\nu]$, which is also equal to $\int_{x \in X} h(x) d \nu$ by the change of variable formula, is equal to $\sup _{i \in I}\left(\int_{x \in X} h(x) d \nu_{i}+\alpha r-\alpha r_{i}\right)$, for every $h \in \mathcal{L}_{\alpha}(X, d)$. This is (8), and this shows that $\nu$ is the naive supremum of $\left(\nu_{i}, r_{i}\right)_{i \in I}$.

The associated linear prevision $G$ is then Scott-continuous, so we can apply Proposition 4.2, item 3: $(\nu, r)$ is the supremum of $\left(\nu_{i}, r_{i}\right)_{i \in I}$.

Theorem 4.15 does not say that $\mathbf{V}_{\leq 1} X$ and $\mathbf{V}_{1} X$ are continuous complete. We will answer this question in Section 8. 
Remark 4.16. The proof of Theorem 4.15 would be valid in the more general situation where $X, d$ is a standard quasi-metric space such that every continuous valuation on its space of formal balls extends to a measure. Hence this would apply to all standard quasi-metric spaces whose spaces of formal balls are LCS-complete in their Scott topology. For instance, this applies to all quasi-continuous complete quasi-metric spaces [27], whose spaces of formal balls are quasi-continuous dcpos, hence locally compact and sober.

\section{Algebraicity for subprobability valuations}

A simple valuation is a finite linear combination $\sum_{i=1}^{n} a_{i} \delta_{x_{i}}$, where $a_{1}, \ldots$, $a_{n} \in \mathbb{R}_{+}$. It is a (simple) subprobability valuation if $\sum_{i=1}^{n} a_{i} \leq 1$, a (simple) probability valuation if $\sum_{i=1}^{n} a_{i}=1$.

For the next lemma, we will need to use some stable compactness results. For more information on stably compact spaces, see Chapter 9 of [16]. Given any topological space $Y$, the coarsest topology that contains the complements of compact saturated subsets of $Y$ is the cocompact topology on $Y$, and the resulting space is written as $Y^{\mathrm{d}}$. The patch topology is the join of the original and the cocompact topology, and $Y^{\text {patch }}$ denotes $Y$ with the patch topology. When $Y$ is stably compact (namely, sober, coherent, locally compact, and compact), then $Y^{\text {patch }}$ is compact Hausdorff. For example, $\overline{\mathbb{R}}_{+}$(with the Scott topology of $\leq$, or equivalent with the $d_{\mathbb{R}}$-Scott topology) is stably compact, the open subsets of $\left(\overline{\mathbb{R}}_{+}\right)^{\mathrm{d}}$ are the half-open intervals $\left[0, a\left[, a \in \mathbb{R}_{+}\right.\right.$, plus the whole space itself, and $\left(\overline{\mathbb{R}}_{+}\right)^{\text {patch }}$ is $\overline{\mathbb{R}}_{+}$with its usual Hausdorff topology.

Lemma 7.3 (4) of [19] states that for every continuous complete quasimetric space $X, d, \mathcal{L}_{\alpha}(X, d)$ and $\mathcal{L}_{\alpha}^{a}(X, d)(a>0)$ are stably compact. Corollary 7.7 (1) of [19] states that, for every finite family of coefficients $a_{1}, \cdots, a_{n} \in$ $\mathbb{R}_{+}$, for all center points $x_{1}, \ldots, x_{n}$ in the continuous complete quasi-metric space $X, d$, the map $h \mapsto \sum_{j=1}^{n} a_{j} h\left(x_{j}\right)$ is continuous from $\mathcal{L}_{\alpha}(X, d)^{\mathrm{d}}$ (resp., $\mathcal{L}_{\alpha}^{a}(X, d)^{\mathrm{d}}$, for any $\left.a>0\right)$ to $\left(\overline{\mathbb{R}}_{+}\right)^{\mathrm{d}}$. We use that to show the following.

Lemma 5.1. Let $X, d$ be a continuous complete quasi-metric space, $\alpha \in \mathbb{R}_{+}$, and $a \in \mathbb{R}_{+}, a>0$. For every $n \in \mathbb{N}$, for all $a_{1}, \ldots, a_{n} \in \mathbb{R}_{+}$and every $n$ tuple of center points $x_{1}, \ldots, x_{n}$ in $X, d$, for every continuous valuation $\nu$ on $X$, the map $h \mapsto \mathrm{d}_{\mathbb{R}}\left(\sum_{j=1}^{n} a_{j} h\left(x_{j}\right), \int_{x \in X} h(x) d \nu\right)$ is continuous from $\mathcal{L}_{\alpha}(X, d)^{\text {patch }}$ (resp., $\left.\mathcal{L}_{\alpha}^{a}(X, d)^{\text {patch }}\right)$ to $\left(\overline{\mathbb{R}}_{+}\right)^{d}$.

Proof. We only deal with $\mathcal{L}_{\alpha}(X, d)$, as the case of $\mathcal{L}_{\alpha}^{a}(X, d)$ is similar. 
Since the patch topology is finer than the cocompact topology, the map $h \mapsto \sum_{j=1}^{n} a_{j} h\left(x_{j}\right)$ is continuous from $\mathcal{L}_{\alpha}(X, d)^{\text {patch }}$ to $\left(\overline{\mathbb{R}}_{+}\right)^{\mathrm{d}}$. The map $h \mapsto \int_{x \in X} h(x) d \nu$ is continuous from $\mathcal{L} X$ to $\overline{\mathbb{R}}_{+}$(every linear prevision is Scott-continuous), hence also from $\mathcal{L}_{\alpha}(X, d)$ to $\overline{\mathbb{R}}_{+}$(because $\mathcal{L}_{\alpha}(X, d)$ has the subspace topology from $\mathcal{L} X)$, hence also from $\mathcal{L}_{\alpha}(X, d)^{\text {patch }}$ to $\overline{\mathbb{R}}_{+}$. The claim will then follow from the fact that $(s, t) \mapsto \mathrm{d}_{\mathbb{R}}(s, t)$ is continuous from $\left(\overline{\mathbb{R}}_{+}\right)^{\mathrm{d}} \times \overline{\mathbb{R}}_{+}$to $\left(\overline{\mathbb{R}}_{+}\right)^{\mathrm{d}}$, which we now prove. The non-trivial open subsets of $\left(\overline{\mathbb{R}}_{+}\right)^{\mathrm{d}}$ are the open intervals $\left[0, a\left[\right.\right.$. The inverse image of the latter by $\mathrm{d}_{\mathbb{R}}$ is $\left\{(s, t) \in \overline{\mathbb{R}}_{+} \times \overline{\mathbb{R}}_{+} \mid s<t+a\right\}=\left\{(s, t) \in \overline{\mathbb{R}}_{+} \times \overline{\mathbb{R}}_{+} \mid \exists b \in \mathbb{R}_{+} . s<b+a, b<\right.$ $t\}=\bigcup_{b \in \mathbb{R}_{+}}[0, b+a[\times] b,+\infty]$.

Lemma 5.2. Let $X, d$ be a continuous complete quasi-metric space.

For all center points $x_{1}, \ldots, x_{n}$ and all non-negative reals $a_{1}, \ldots, a_{n}$ with $\sum_{i=1}^{n} a_{i} \leq 1$, the simple valuation $\sum_{i=1}^{n} a_{i} \delta_{x_{i}}$ is a center point of $\mathbf{V}_{\leq 1} X, d_{K R}$ (resp., of $\mathbf{V}_{1}, d_{K R}$ if additionally $\sum_{i=1}^{n} a_{i}=1$ ).

The same result holds with $d_{K R}^{a}$ instead of $d_{K R}$, for any $a \in \mathbb{R}_{+}, a>0$.

Proof. We only deal with the case of $\mathbf{V}_{\leq 1} X$, and of $d_{\mathrm{KR}}$, the other cases are similar. Let $\nu_{0}=\sum_{i=1}^{n} a_{i} \delta_{x_{i}}, U$ be the open ball $B_{\left(\nu_{0}, 0\right),<\epsilon}^{d_{\mathrm{KR}}^{+}} . U$ is upwardsclosed: if $(\nu, r) \leq d_{\mathrm{KR}}^{+}\left(\nu^{\prime}, r^{\prime}\right)$ and $(\nu, r) \in U$, then $d_{\mathrm{KR}}\left(\nu, \nu^{\prime}\right) \leq r-r^{\prime}$ and $d_{\mathrm{KR}}\left(\nu_{0}, \nu\right)<\epsilon-r$, so $d_{\mathrm{KR}}\left(\nu_{0}, \nu^{\prime}\right)<\epsilon-r^{\prime}$ by the triangular inequality, and that means that $\left(\nu^{\prime}, r^{\prime}\right)$ is in $U$.

Recall that $\mathbf{V}_{\leq 1}, d_{\mathrm{KR}}$ is complete, and that directed suprema of formal balls are computed as naive suprema, by Theorem 4.15. To show that $U$ is Scott-open, we consider a directed family $\left(\nu_{i}, r_{i}\right)_{i \in I}$ in $\mathbf{B}\left(\mathbf{V}_{\leq 1} X, d_{\mathrm{KR}}\right)$, with supremum $(\nu, r)$, and we assume that $(\nu, r)$ is in $U$. That supremum is given as in (8). Hence $r=\inf _{i \in I} r_{i}$ and for every $h \in \mathcal{L}_{1}(X, d), \int_{x \in X} h(x) d \nu=$ $\sup _{i \in I}\left(\int_{x \in X} h(x) d \nu_{i}+r-r_{i}\right)$. (In the case of $d_{\mathrm{KR}}^{a}$, the same formula holds, this time for every $h \in \mathcal{L}_{1}^{a}(X, d)$.) Since $(\nu, r) \in U, d_{\mathrm{KR}}\left(\nu_{0}, \nu\right)<\epsilon-r$. In particular, $\epsilon>r$ and $\int_{x \in X} h(x) d \nu_{0}-\epsilon+r<\int_{x \in X} h(x) d \nu$ for every $h \in \mathcal{L}_{1}(X, d)$ (resp., $\left.\mathcal{L}_{1}^{a}(X, d)\right)$. Therefore, for every $h \in \mathcal{L}_{1}(X, d)$ (resp., $\mathcal{L}_{1}^{a}(X, d)$ ), there is an index $i \in I$ such that $\int_{x \in X} h(x) d \nu_{0}-\epsilon+r<\int_{x \in X} h(x) d \nu_{i}+r-r_{i}$, or equivalently,

$$
\sum_{j=1}^{n} a_{j} h\left(x_{j}\right)<\int_{x \in X} h(x) d \nu_{i}+\epsilon-r_{i} .
$$

Moreover, since $\epsilon>r=\inf _{i \in I} r_{i}$, we may take $i$ so large that $\epsilon-r_{i}>0$. 
Let $V_{i}$ be the set of all $h \in \mathcal{L}_{1}(X, d)$ (resp., $\mathcal{L}_{1}^{a}(X, d)$ ) satisfying (9). We have just shown that $\mathcal{L}_{1}(X, d)$ (resp., $\mathcal{L}_{1}^{a}(X, d)$ ) is included in $\bigcup_{i \in I} V_{i}$. $V_{i}$ is also the inverse image of $\left[0, \epsilon-r_{i}\right.$ [ by the map $h \mapsto \mathbb{d}_{\mathbb{R}}\left(\sum_{j=1}^{n} a_{j} h\left(x_{j}\right), \int_{x \in X} h(x) d \nu_{i}\right)$, which is continuous from $\mathcal{L}_{1}(X, d)^{\text {patch }}$ (resp., $\left.\mathcal{L}_{1}^{a}(X, d)^{\text {patch }}\right)$ to $\left(\overline{\mathbb{R}}_{+}\right)^{\text {d }}$ by Lemma 5.1. Therefore $V_{i}$ is open in $\mathcal{L}_{1}(X, d)^{\text {patch }}$ (resp., $\left.\mathcal{L}_{1}^{a}(X, d)^{\text {patch }}\right)$, itself a compact space; remember indeed that $\mathcal{L}_{1}(X, d)$ and $\mathcal{L}_{1}^{a}(X, d)$ are stably compact [19, Lemma 7.3 (4)], as we have said near the beginning of the current section.

Hence there is a finite subset $J$ of $I$ such that $\left(V_{i}\right)_{i \in J}$ is also an open cover of $\mathcal{L}_{1}(X, d)^{\text {patch }}$ (resp., $\mathcal{L}_{1}^{a}(X, d)$ ). That means that for every $h \in \mathcal{L}_{1}(X, d)$ (resp., $\mathcal{L}_{1}^{a}(X, d)$ ), there is an index $i \in J$ (not just in $I$ ) such that (9) holds. By directedness, there is a single index $i \in I$ such that (9) holds for every $h \in \mathcal{L}_{1}(X, d)$ (resp., $\mathcal{L}_{1}^{a}(X, d)$ ). That implies that $\left(\nu_{i}, r_{i}\right)$ is in $U$, proving the claim.

Remark 5.3. Using the same proof, but relying on Remark 4.4 instead of Theorem 4.15, we obtain that simple valuations supported on center points are center points of $\mathbf{V} X, d_{K R}$ (and similarly for subnormalized, resp. normalized valuations, and for $d_{K R}^{a}$ instead of $d_{K R}$ ), under the alternative assumption that $X, d$ is standard and Lipschitz regular.

We have almost everything we need for our proof of algebraicity for spaces of valuations (Theorem 5.7)... except for the following three lemmata. For every continuous dcpo $Y$, we recall that $\mathbf{V}_{Y}$ and $\mathbf{V}_{\leq 1} Y$ are continuous dcpos again. Moreover, a basis is given by simple valuations [21] (see also [13, Theorem IV-9.16]). The following lemma is an easy consequence of this fact, and is implicit in [13, Exercise IV-9.29]; we can in fact even require every $a_{i}$ to be rational.

Lemma 5.4. Let $Y$ be a continuous dcpo, with a basis $\mathcal{B}$. VY (resp., $\mathbf{V}_{\leq 1} Y$ ) is a continuous dcpo, and a basis is given by simple valuations supported on $\mathcal{B}$, viz., of the form $\sum_{i=1}^{n} a_{i} \delta_{y_{i}}$ with $y_{i} \in \mathcal{B}$ (resp., and with $\sum_{i=1}^{n} a_{i} \leq 1$ ).

For every $h \in \mathcal{L}_{1}(X, d)$, and assuming again that $X, d$ is standard, we have said in the preliminaries that the map $h^{\prime}:(x, r) \mapsto h(x)-r$ is Scottcontinuous from $\mathbf{B}(X, d)$ to $\mathbb{R} \cup\{+\infty\}$. It follows that $h^{\prime \prime}:(x, r) \mapsto \max (h(x)-$ $r, 0)$ is Scott-continuous from $\mathbf{B}(X, d)$ to $\overline{\mathbb{R}}_{+}$. Then we have: 
Lemma 5.5. Let $X, d$ be a standard quasi-metric space, $\nu$ be a continuous valuation on $X$, and $h \in \mathcal{L}_{1}(X, d)$. Define $h^{\prime \prime}(x, r)=\max (h(x)-r, 0)$. Then:

$$
\int_{(x, r) \in \mathbf{B}(X, d)} h^{\prime \prime}(x, r) d \eta_{X}[\nu]=\int_{x \in X} h(x) d \nu .
$$

Proof. $\int_{(x, r) \in \mathbf{B}(X, d)} h^{\prime \prime}(x, r) d \eta_{X}[\nu]=\int_{x \in X} h^{\prime \prime}\left(\eta_{X}(x)\right) d \nu$, by the change of variable formula. We conclude since $h^{\prime \prime}\left(\eta_{X}(x)\right)=h^{\prime \prime}(x, 0)=h(x)$ for every $x \in X$.

A strong basis of a standard quasi-metric space $X, d$ is any set $\mathcal{B}$ of center points of $X$ such that, for every $x \in X,(x, 0)$ is the supremum of a directed family of formal balls with center points in $\mathcal{B}$ (see Definition 7.4 .66 of [16], which is stated with $d$-finite points and Cauchy nets instead of center points and directed families of formal balls). The standard space $X, d$ is algebraic if and only if it has a strong basis. The largest strong basis is simply the set of all center points.

Lemma 5.6. Let $X, d$ be a standard algebraic space, with a strong basis $\mathcal{B}$. Then $\mathbf{B}(X, d)$ is a continuous dcpo, with a basis consisting of the formal balls $(x, r)$ with $x \in \mathcal{B}$. For $x \in \mathcal{B},(x, r) \ll(y, s)$ if and only if $d(x, y)<r-s$.

Proof. Since $X, d$ is standard algebraic hence continuous, $\mathbf{B}(X, d)$ is a continuous poset. We also recall that $(x, r) \ll(y, s)$ if and only if $d(x, y)<r-s$, whenever $x$ is a center point. Now let $(y, s) \in \mathbf{B}(X, d)$. We write $(y, s)$ as the supremum of a directed family $\left(y_{i}, s_{i}\right)_{i \in I}$ of formal balls way-below $(y, s)$. By assumption $\left(y_{i}, 0\right)$ is the supremum of a directed family $\left(x_{i j}, r_{i j}\right)_{j \in J_{i}}$, where $x_{i j} \in \mathcal{B}$. Since $X, d$ is standard, $\left(y_{i}, s_{i}\right)$ is the supremum of the directed family $\left(x_{i j}, s_{i}+r_{i j}\right)_{j \in J_{i}}$. The family $\left(x_{i j}, s_{i}+r_{i j}+1 / 2^{n}\right)_{j \in J_{i}, n \in \mathbb{N}}$ is also directed, and its supremum is also equal to $\left(y_{i}, s_{i}\right)$, as one easily checks by looking at the upper bounds of the family. Additionally, since $\left(x_{i j}, s_{i}+r_{i j}\right) \leq d^{d^{+}}\left(y_{i}, s_{i}\right)$, we have $d\left(x_{i j}, y_{i}\right) \leq r_{i j}<r_{i j}+1 / 2^{n}$, so each $\left(x_{i j}, s_{i}+r_{i j}+1 / 2^{n}\right)$ is way-below $\left(y_{i}, s_{i}\right)$. This is exactly what we need to conclude that $\left(x_{i j}, s_{i}+r_{i j}+1 / 2^{n}\right)_{i \in I, j \in J_{i}, n \in \mathbb{N}}$ is a directed family whose supremum is $(y, s)$ : if, in a poset, $a$ is the supremum of a directed family $D$ and each element $b$ of $D$ is the supremum of a directed family $D_{b}$ of elements way-below $b$, then $\bigcup_{b \in D} D_{b}$ is a directed family whose supremum is $a$ (see Exercise 5.1.13 of [16] for example).

Theorem 5.7 (Algebraicity for spaces of subprobabilities). Let $X, d$ be an algebraic complete quasi-metric space, with a strong basis $\mathcal{B}$. 
The spaces $\mathbf{V}_{\leq 1} X, d_{K R}$ and $\mathbf{V}_{\leq 1} X, d_{K R}^{a}$ (for any $a \in \mathbb{R}_{+}, a>0$ ) are algebraic complete, and a strong basis is given by the simple valuations $\sum_{j=1}^{n} a_{j} \delta_{x_{j}}$ with $x_{j} \in \mathcal{B}$, and $\sum_{j=1}^{n} a_{j} \leq 1$.

Proof. By Lemma 5.2, all simple subprobability valuations $\sum_{j=1}^{n} a_{j} \delta_{x_{j}}$ such that every $x_{j}$ is a center point in $X$ are center points. Let $\nu \in \mathbf{V}_{\leq 1} X$. We wish to show that $(\nu, 0)$ is the supremum of a directed family of formal balls $\left(\stackrel{\circ}{\nu}_{i}, R\left(\nu_{i}\right)\right)$ below $(\nu, 0)$, and where each $\stackrel{\circ}{\nu}_{i}$ is a simple valuation of the form $\sum_{j=1}^{n} a_{j} \delta_{x_{j}}$, with $x_{j} \in \mathcal{B}$, and $\sum_{j=1}^{n} a_{j} \leq 1$.

Finding $\stackrel{\circ}{\nu}_{i}$ and $R\left(\nu_{i}\right)$ is obvious if $\nu$ is the zero valuation, so we assume for the rest of the proof that $\nu(X) \neq 0$.

Since $X, d$ is algebraic complete, $\mathbf{B}(X, d)$ is a continuous dcpo and the formal balls with centers in $\mathcal{B}$ are a basis, by Lemma 5.6.

We profit from the fact that, since $\mathbf{B}(X, d)$ is a continuous poset, $\mathbf{V}_{\leq 1}(\mathbf{B}(X, d))$ is a continuous dcpo. Lemma 5.4 even allows us to say that a basis of the latter consists in the simple valuations $\mu=\sum_{j=1}^{n} a_{j} \delta_{\left(x_{j}, r_{j}\right)}$, where each $x_{j}$ is in $\mathcal{B}$, and $\sum_{j=1}^{n_{i}} a_{j} \leq 1$. In particular, for every $\nu \in \mathbf{V}_{\leq 1} X, \eta_{X}[\nu]$ is the supremum of a directed family of simple valuations $\nu_{i}=\sum_{j=1}^{n_{i}} a_{i j} \delta_{\left(x_{i j}, r_{i j}\right)}$, $i \in I$, where each $x_{i j}$ is a center point, and $\sum_{j=1}^{n_{i}} a_{i j} \leq 1$.

We can require that $r_{i j}<1$ for all $i, j$, by the following argument. Define $\nu_{i}^{\prime}(U)=\nu_{i}\left(U \cap V_{1}\right)$, where $V_{1}=\{(x, r) \in \mathbf{B}(X, d) \mid r<1\} . V_{1}$ is open, since $X, d$ is standard. $\nu_{i}^{\prime}$ is the restriction of $\nu_{i}$ to $V_{1}$, and is again a subprobability valuation. Explicitly, $\nu_{i}^{\prime}=\sum_{\substack{1 \leq j \leq n_{i} \\ r_{i j}<1}} a_{i j} \delta_{\left(x_{i j}, r_{i j}\right)}$. Note that all the radii involved in the latter sum are strictly less than 1 . If $\nu_{i} \leq \nu_{i^{\prime}}$ then $\nu_{i}^{\prime} \leq \nu_{i^{\prime}}^{\prime}$, so the family $\left(\nu_{i}^{\prime}\right)_{i \in I}$ is directed as well. Since $\nu_{i}^{\prime} \leq \nu_{i} \ll \eta_{X}[\nu]$, this is a family of (subprobability) simple valuations way-below $\eta_{X}[\nu]$. Moreover, for every open subset $U$ of $\mathbf{B}(X, d), \eta_{X}[\nu](U)=\nu(U \cap X)=\nu\left(U \cap V_{1} \cap X\right)=$ $\eta_{X}[\nu]\left(U \cap V_{1}\right)$ is equal to $\sup _{i \in I} \nu_{i}\left(U \cap V_{1}\right)=\sup _{i \in I} \nu_{i}^{\prime}(U)$, so $\eta_{X}[\nu]$ is also the supremum of $\left(\nu_{i}^{\prime}\right)_{i \in I}$. All this concurs to show that we may assume that $\nu_{i}$ satisfies $r_{i j}<1$ for all $i, j$, replacing $\nu_{i}$ by $\nu_{i}^{\prime}$ if needed.

For every simple subprobability valuation $\mu=\sum_{j=1}^{n} a_{j} \delta_{\left(x_{j}, r_{j}\right)}$ on $\mathbf{B}(X, d)$ such that $\mu \leq \eta_{X}[\nu]$ and with $r_{j}<1$ for every $j$, let:

$$
\begin{aligned}
\stackrel{\circ}{\mu} & =\sum_{i=1}^{n} a_{j} \delta_{x_{j}} \\
R(\mu) & =\sum_{j=1}^{n} a_{j} r_{j}+\nu(X)-\sum_{j=1}^{n} a_{j} .
\end{aligned}
$$


$R(\mu)$ is a non-negative number, owing to the fact that $\mu \leq \eta_{X}[\nu]$ : indeed $\sum_{j=1}^{n} a_{j}=\mu(\mathbf{B}(X, d)) \leq \eta_{X}[\nu](\mathbf{B}(X, d))=\nu(X)$. Therefore $\beta(\mu)=$ $(\stackrel{\rho}{\mu}, R(\mu))$ is a well-defined formal ball on $\mathbf{V}_{\leq 1} X, d_{\mathrm{KR}}$ (resp., $\left.d_{\mathrm{KR}}^{a}\right)$.

For every $h \in \mathcal{L}_{1}(X, d)$ (resp., $\mathcal{L}_{1}^{a}(X, d)$ ), recall the construction $h^{\prime \prime}$ mentioned in Lemma 5.5. However, apply it to $h+\mathbf{1}$, not $h$. In other words, $(h+\mathbf{1})^{\prime \prime}$ maps $(x, r)$ to $\max (h(x)-r+1,0)$. We have $\int_{(x, r) \in \mathbf{B}(X, d)}(h+$ 1) $)^{\prime \prime}(x, r) d \mu=\sum_{j=1}^{n} a_{j}\left(h\left(x_{j}\right)-r_{j}+1\right)$, because $r_{j}<1$ for all $i, j$. Applying this to $\mu=\nu_{i}$ and $\mu=\nu_{i^{\prime}}$, we obtain that if $\nu_{i} \leq \nu_{i^{\prime}}$, then $\int_{(x, r) \in \mathbf{B}(X, d)}(h+$ 1)" $(x, r) d \nu_{i}=\sum_{j=1}^{n_{i}} a_{i j} h\left(x_{i j}\right)-\sum_{j=1}^{n_{i}} a_{i j} r_{j}+\sum_{j=1}^{n_{i}} a_{i j}$ is less than or equal to $\int_{(x, r) \in \mathbf{B}(X, d)}(h+\mathbf{1})^{\prime \prime}(x, r) d \nu_{i^{\prime}}=\sum_{j=1}^{n_{i^{\prime}}} a_{i^{\prime} j} h\left(x_{i^{\prime} j}\right)-\sum_{j=1}^{n_{i^{\prime}}} a_{i^{\prime} j} r_{j}+\sum_{j=1}^{n_{i^{\prime}}} a_{i^{\prime} j}$. In other words, $\sum_{j=1}^{n_{i}} a_{i j} h\left(x_{i j}\right) \leq \sum_{j=1}^{n_{i^{\prime}}} a_{i^{\prime} j} h\left(x_{i^{\prime} j}\right)+R\left(\nu_{i}\right)-R\left(\nu_{i^{\prime}}\right)$. This can be rewritten as $\mathrm{d}_{\mathbb{R}}\left(\int_{x \in X} h(x) d \stackrel{\circ}{\nu}_{i}, \int_{x \in X} h(x) d{\stackrel{\circ}{\nu^{\prime}}}^{\prime}\right) \leq R\left(\nu_{i}\right)-R\left(\nu_{i^{\prime}}\right)$. Since $h$ is arbitrary, $\beta\left(\nu_{i}\right) \leq d_{\mathrm{KR}}^{+} \beta\left(\nu_{i^{\prime}}\right)$ (resp., $\leq d_{\mathrm{KR}}^{a+}$, by multiplying $h^{\prime \prime}$ by $1 / a$ first). This shows that the family $\left(\beta\left(\nu_{i}\right)\right)_{i \in I}$ is directed.

We now claim that $\beta\left(\nu_{i}\right) \leq d_{\mathrm{KR}}^{+}(\nu, 0)$ for every $i \in I$ (resp., $\left.\leq d_{\mathrm{KR}}^{a+}\right)$. Fix $h \in \mathcal{L}_{1}(X, d)$ (resp., in $\mathcal{L}_{1}^{a}(X, d)$ ). We wish to show that $\sum_{j=1}^{n_{i}} a_{i j} h\left(x_{i j}\right) \leq$ $\int_{x \in X} h(x) d \nu+R\left(\nu_{i}\right)$. To this end, recall that $\nu_{i} \ll \eta_{X}[\nu]$, in particular $\nu_{i} \leq \eta_{X}[\nu]$. We integrate $(h+\mathbf{1})^{\prime \prime}$ with respect to each side of the inequality: $\int_{(x, r) \in \mathbf{B}(X, d)}(h+\mathbf{1})^{\prime \prime}(x, r) d \nu_{i}=\sum_{j=1}^{n_{i}} a_{i j} h\left(x_{i j}\right)-\sum_{j=1}^{n_{i}} a_{i j} r_{i j}+\sum_{j=1}^{n_{i}} a_{i j}$, and $\int_{(x, r) \in \mathbf{B}(X, d)}(h+\mathbf{1})^{\prime \prime}(x, r) d \eta_{X}[\nu]=\int_{x \in X} h(x) d \nu+\nu(X)$, by Lemma 5.5. Therefore $\sum_{j=1}^{n_{i}} a_{i j} h\left(x_{i j}\right) \leq \int_{x \in X} h(x) d \nu+\nu(X)+\sum_{j=1}^{n_{i}} a_{i j} r_{i j}-\sum_{j=1}^{n_{i}} a_{i j}=$ $\int_{x \in X} h(x) d \nu+R\left(\nu_{i}\right)$.

We finally claim that $\sup _{i \in I} \beta\left(\nu_{i}\right)=(\nu, 0)$. Let $R=\inf _{i \in I} R\left(\nu_{i}\right)$. Let $h=$ $\mathbf{1}$, and let us compute $\int_{(x, r) \in \mathbf{B}(X, d)} h^{\prime \prime}(x, r) d \nu_{i}=\sum_{j=1}^{n_{i}} a_{i j}\left(1-r_{i j}\right)=\nu(X)-$ $R\left(\nu_{i}\right)$. Since $\sup _{i \in I} \nu_{i}=\eta_{X}[\nu]$ and integration is Scott-continuous in the valuation, $\sup _{i \in I}\left(\nu(X)-R\left(\nu_{i}\right)\right)=\int_{(x, r) \in \mathbf{B}(X, d)} h^{\prime \prime}(x, r) d \eta_{X}[\nu]=\int_{x \in X} h(x) d \nu=$ $\nu(X)$. Hence $R=0$.

By Theorem 4.15, directed suprema are computed as naive suprema. That is to say, $\sup _{i \in I} \beta\left(\nu_{i}\right)$ is a formal ball $(G, R)$, with $R=0$ as we have just seen, and where (equating $G$ with a linear prevision) $G$ maps every $h \in \mathcal{L}_{1}(X, d)$ (resp., $\left.\mathcal{L}_{1}^{a}(X, d)\right)$ to $\sup _{i \in I}\left(\sum_{j=1}^{n_{i}} a_{i j} h\left(x_{i j}\right)-R\left(\nu_{i}\right)\right)$. We have already noticed that $\int_{(x, r) \in \mathbf{B}(X, d)}(h+\mathbf{1})^{\prime \prime}(x, r) d \nu_{i}$ is equal to $\sum_{j=1}^{n_{i}} a_{i j} h\left(x_{i j}\right)-$ $\sum_{j=1}^{n_{i}} a_{i j} r_{i j}+\sum_{j=1}^{n_{i}} a_{i j}$, that is, to $\sum_{j=1}^{n_{i}} a_{i j} h\left(x_{i j}\right)+\nu(X)-R\left(\nu_{i}\right)$, so $G(h)=$ $\left.\sup _{i \in I} \int_{(x, r) \in \mathbf{B}(X, d)}(h+\mathbf{1})^{\prime \prime}(x, r) d \nu_{i}\right)-\nu(X)$. This is equal to $\int_{(x, r) \in \mathbf{B}(X, d)}(h+$ $\mathbf{1})^{\prime \prime}(x, r) d \eta_{X}[\nu]-\nu(X)$, since $\sup _{i \in I} \nu_{i}=\eta_{X}[\nu]$ and integration is Scott- 
continuous in the valuation. In turn, this is equal to $\int_{x \in X}(h+\mathbf{1})(x) d \nu-\nu(X)$ by Lemma 5.5, namely to $\int_{x \in X} h(x) d \nu$. It follows that $G(h)=\int_{x \in X} h(x) d \nu$ for every $h \in \mathcal{L}_{1}(X, d)$ (resp., $\mathcal{L}_{1}^{a}(X, d)$ ), and this suffices to show that $G$ coincides with $h \mapsto \int_{x \in X} h(x) d \nu$, by Corollary 3.14 (resp., Corollary 3.19).

Remark 5.8. For a poset $X, \mathbf{B}\left(X, d_{\leq}\right)$is order-isomorphic to $\left.\left.X \times\right]-\infty, 0\right]$ [20, Example 1.6]. Hence $X, d_{\leq}$is a continuous complete quasi-metric space if and only if $X$ is a continuous dcpo, and in that case $(x, r) \ll(y, s)$ in $\mathbf{B}(X, d)$ if and only if $x \ll y$ and $r>s$. For every $x \in X$ and $\epsilon>0$, $B_{(x, 0), \epsilon}^{d_{\leq}^{+}}=\left\{(y, s) \mid d_{\leq}(x, y)+s<\epsilon\right\}=\{(y, s) \mid x \leq y, s<\epsilon\}$ : if $x$ is finite (namely, $x \ll x)$, then this is equal to $\uparrow(x, \epsilon)$, which is Scott-open: so $x$ is a center point of $X, d_{\leq}$. If $X$ is an algebraic dcpo, every point $x \in X$ is a supremum of a directed family $\left(x_{i}\right)_{i \in I}$ of finite points of $X$, so $(x, 0)$ is also the supremum of the family $\left(x_{i}, 0\right)_{i \in I}$, where, as we have seen, each $x_{i}$ is a center point. Therefore, for every algebraic dcpo $X$, the quasi-metric space $X, d_{\leq}$is an algebraic complete quasi-metric space.

In that case, $\mathbf{V}_{\leq 1} X, d_{K R}^{a}$ is algebraic complete. When $a=1$, and in light of Remark 3.6, this states that $\mathbf{V}_{\leq 1} X$ is an algebraic complete quasimetric space in Sünderhauf's sup quasi-metric, allowing us to retrieve his main result [29, Theorem 3.4]-in the case of subprobability valuations.

\section{Algebraicity for probability valuations}

The situation is less neat than with subprobability valuations (Theorem 5.7) or with metric spaces, and we shall distinguish two settings where we can conclude: for the bounded $d_{\mathrm{KR}}^{a}$ quasi-metrics (Theorem 6.1), or for $d_{\mathrm{KR}}$ assuming the existence of a so-called root in $X$ (Theorem 6.6).

Theorem 6.1 (Algebraicity for spaces of probabilities, $d_{\mathbf{K R}}^{a}$ ). Let $X, d$ be an algebraic complete quasi-metric space, with a strong basis $\mathcal{B}$.

The space $\mathbf{V}_{1} X, d_{K R}^{a}$ is algebraic complete, for every $a \in \mathbb{R}_{+}, a>0$.

All the simple probability valuations $\sum_{i=1}^{n} a_{i} \delta_{x_{i}}$ with $x_{i}$ center points in $X, d, \sum_{i=1}^{n} a_{i}=1$, are center points, and form a strong basis, even when each $x_{i}$ is taken from $\mathcal{B}$.

Proof. Let $\nu \in \mathbf{V}_{1} X$. We wish to exhibit a directed family of formal balls $\left(\nu_{i}^{\prime}, r_{i}^{\prime}\right), i \in I$, below $(\nu, 0)$, whose supremum is $(\nu, 0)$, where each $\nu_{i}^{\prime}$ is a simple valuation of the form $\sum_{j=1}^{n} a_{j} \delta_{x_{j}}$, with $x_{j}$ in $\mathcal{B}$, and $\sum_{j=1}^{n} a_{j}=1$. 
As in the proof of Theorem 5.7, $\eta_{X}[\nu]$ is the supremum of a directed family of simple valuations $\nu_{i}=\sum_{j=1}^{n_{i}} a_{i j} \delta_{\left(x_{i j}, r_{i j}\right)}, i \in I$, where each $x_{i j}$ is in $\mathcal{B}$, and $\sum_{j=1}^{n_{i}} a_{i j} \leq 1$.

We can require that $r_{i j}<1$ for all $i, j$, as in the proof of Theorem 5.7, and we similarly define $\stackrel{\circ}{\nu}_{i}$ as $\sum_{j=1}^{n_{i}} a_{i j} \delta_{x_{i j}}, R\left(\nu_{i}\right)=\sum_{j=1}^{n_{i}} a_{i j} r_{i j}+1-\sum_{j=1}^{n_{i}} a_{i j}$, $\beta\left(\nu_{i}\right)=\left(\stackrel{\circ}{i}_{i}, R\left(\nu_{i}\right)\right)$. Define $i \preceq i^{\prime}$ if and only if $\nu_{i} \leq \nu_{i^{\prime}}$, for all $i, i^{\prime} \in I$. As before, we show that:

(*) for all $i \preceq i^{\prime}$ in $I, \beta\left(\nu_{i}\right) \leq d_{\mathrm{KR}}^{a+} \beta\left(\nu_{i^{\prime}}\right)$.

This implies that the family $\left(\beta\left(\nu_{i}\right)\right)_{i \in I}$ is directed. We also show that $\beta\left(\nu_{i}\right) \leq d_{\mathrm{KR}}^{a+}$ $(\nu, 0)$ for every $i \in I$, and that $\sup _{i \in I} \beta\left(\nu_{i}\right)=(\nu, 0)$. However $\stackrel{\circ}{i}_{i}$ is not a probability, only a subprobability valuation.

Let us fix a center point $x_{0}$. (There must exist one: the only algebraic quasi-metric space without a center point is the empty space, and if $X$ is empty then $\mathbf{V}_{1} X$ is empty as well, so we would not have had a $\nu \in \mathbf{V}_{1} X$ to start with.) For each $i \in I$, let $\lambda_{i}=1-\stackrel{\circ}{\nu}_{i}(X), \nu_{i}^{\prime}=\stackrel{\circ}{\nu}_{i}+\lambda_{i} \delta_{x_{0}}$, and $r_{i}^{\prime}=R\left(\nu_{i}\right)+a \lambda_{i}$. Now $\nu_{i}^{\prime}$ is a probability valuation.

We check that $\left(\nu_{i}^{\prime}, r_{i}^{\prime}\right)_{i \in I}$ is directed. To this end, it is enough to show that for all $i \preceq i^{\prime}$ in $I,\left(\nu_{i}^{\prime}, r_{i}^{\prime}\right) \leq d_{\mathrm{KR}}^{a+}\left(\nu_{i^{\prime}}^{\prime}, r_{i^{\prime}}^{\prime}\right)$. Before we do so, we note that if $i \preceq i^{\prime}$, then $\nu_{i}(\mathbf{B}(X, d)) \leq \nu_{i^{\prime}}(\mathbf{B}(X, d))$, that is, $\sum_{j=1}^{n_{i}} a_{i j} \leq \sum_{j=1}^{n_{i^{\prime}}} a_{i^{\prime} j}$; equivalently, $\stackrel{\circ}{\nu}_{i}(X) \leq{\stackrel{\circ}{i^{\prime}}}^{\prime}(X)$. Therefore:

$$
(* *) \text { for all } i \preceq i^{\prime} \text { in } I, \lambda_{i} \geq \lambda_{i^{\prime}} \text {. }
$$

We also note that for all $i \preceq i^{\prime}$ in $I$, since by $(*) d_{\mathrm{KR}}^{a}\left(\stackrel{\circ}{\nu_{i}}, \stackrel{\circ}{i^{\prime}}\right) \leq R\left(\nu_{i}\right)-R\left(\nu_{i^{\prime}}\right)$, the inequality $\int_{x \in X} h(x) d \stackrel{\circ}{\nu}_{i} \leq \int_{x \in X} h(x) d{\stackrel{\circ}{i^{\prime}}}+R\left(\nu_{i}\right)-R\left(\nu_{i^{\prime}}\right)$ holds for $h$ equal to the constant fonction equal to $a$; hence $a \stackrel{\circ}{\nu}_{i}(X) \leq a{\stackrel{\circ}{i^{\prime}}}^{\prime}(X)+R\left(\nu_{i}\right)-R\left(\nu_{i^{\prime}}\right)$. This implies:

$$
(* * *) \text { for all } i \preceq i^{\prime} \text { in } I, r_{i}^{\prime} \geq r_{i^{\prime}}^{\prime}
$$

Let now $h$ be an arbitrary element from $\mathcal{L}_{1}^{a}(X, d)$, and assume $i \preceq i^{\prime}$. We 
have the following:

$$
\begin{aligned}
& \mathrm{d}_{\mathbb{R}}\left(\int_{x \in X} h(x) d \nu_{i}^{\prime}, \int_{x \in X} h(x) d \nu_{i^{\prime}}^{\prime}\right) \\
& =\max \left(\int_{x \in X} h(x) d \stackrel{\circ}{\nu}_{i}+h\left(x_{0}\right) \lambda_{i}-\int_{x \in X} h(x) d{\stackrel{\circ}{i^{\prime}}}-h\left(x_{0}\right) \lambda_{i^{\prime}}, 0\right) \\
& \leq \max \left(R\left(\nu_{i}\right)-R\left(\nu_{i^{\prime}}\right)+h\left(x_{0}\right)\left(\lambda_{i}-\lambda_{i^{\prime}}\right), 0\right) \\
& \quad \text { since } d_{\mathrm{KR}}^{a}\left(\stackrel{\circ}{\nu}_{i},{\stackrel{\circ}{i^{\prime}}}^{\prime}\right) \leq R\left(\nu_{i}\right)-R\left(\nu_{i^{\prime}}\right), \operatorname{using}(*) \\
& \leq \max \left(R\left(\nu_{i}\right)-R\left(\nu_{i^{\prime}}\right)+a\left(\lambda_{i}-\lambda_{i^{\prime}}\right), 0\right) \\
& \quad \quad \operatorname{since} h\left(x_{0}\right) \leq a, \text { and } \lambda_{i} \geq \lambda_{i^{\prime}} \text { by }(* *) \\
& =\max \left(r_{i}^{\prime}-r_{i^{\prime}}^{\prime}, 0\right)=r_{i}^{\prime}-r_{i^{\prime}}^{\prime} \quad \text { by }(* * *) .
\end{aligned}
$$

This shows that for all $i \preceq i^{\prime}$ in $I, d_{\mathrm{KR}}^{a}\left(\nu_{i}^{\prime}, \nu_{i^{\prime}}^{\prime}\right) \leq r_{i}^{\prime}-r_{i^{\prime}}^{\prime}$, hence that $\left(\nu_{i}^{\prime}, r_{i}^{\prime}\right) \leq d_{\mathrm{KR}}^{a+}\left(\nu_{i^{\prime}}^{\prime}, r_{i^{\prime}}^{\prime}\right)$. In particular, $\left(\nu_{i}^{\prime}, r_{i}^{\prime}\right)_{i \in I}$ is directed.

We know that $\beta\left(\nu_{i}\right) \leq d_{\mathrm{KR}}^{a+}(\nu, 0)$ for every $i \in I$, and we need to show that $\left(\nu_{i}^{\prime}, r_{i}^{\prime}\right) \leq d_{\mathrm{KR}}^{a+}(\nu, 0)$ as well. For every $h \in \mathcal{L}_{1}^{a}(X, d)$, this means showing that $\int_{x \in X} h(x) d \dot{\nu}_{i}+h\left(x_{0}\right) \lambda_{i} \leq \int_{x \in X} h(x) d \nu+R\left(\nu_{i}\right)+a \lambda_{i}$. We know that $\int_{x \in X} h(x) d \stackrel{\circ}{\nu}_{i} \leq \int_{x \in X} h(x) d \nu+R\left(\nu_{i}\right)$ since $\beta\left(\nu_{i}\right) \leq d_{\mathrm{KR}}^{a+}(\nu, 0)$, and we conclude since $h\left(x_{0}\right) \leq a$.

Finally, we know that $(\nu, 0)$ is the supremum of the directed family $\left(\beta\left(\nu_{i}\right)\right)_{i \in I}$ in $\mathbf{B}\left(\mathbf{V}_{\leq 1} X, d_{\mathrm{KR}}^{a}\right)$, and we must show that it is also the supremum of the directed family $\left(\nu_{i}^{\prime}, r_{i}^{\prime}\right)_{i \in I}$ in $\mathbf{B}\left(\mathbf{V}_{1} X, d_{\mathrm{KR}}^{a}\right)$.

We claim that:

$$
(\dagger) \inf _{i \in I} \lambda_{i}=0
$$

Indeed, recall that $\sup _{i \in I} \beta\left(\nu_{i}\right)=(\nu, 0)$. Since directed suprema are computed as naive suprema (Theorem 4.15), this means that $\inf _{i \in I} R\left(\nu_{i}\right)=0$, and that $\int_{x \in X} h(x) d \nu$ is equal to $\sup _{i \in I}\left(\int_{x \in X} h(x) d \stackrel{\circ}{\nu}_{i}-R\left(\nu_{i}\right)\right)$ for every $h \in$ $\mathcal{L}_{1}^{a}(X, d)$. Taking $h=a . \mathbf{1}$, the latter yields $a=\sup _{i \in I}\left(a \dot{\nu}_{i}(X)-R\left(\nu_{i}\right)\right)$, or equivalently $\sup _{i \in I}\left(-a \lambda_{i}-R\left(\nu_{i}\right)\right)=0$. Since $\left(-R\left(\nu_{i}\right)\right)_{i \in I}$ is a directed family (because $i \preceq i^{\prime}$ implies $\beta\left(\nu_{i}\right) \leq \beta\left(\nu_{i^{\prime}}\right)$, hence $R\left(\nu_{i}\right) \geq R\left(\nu_{i^{\prime}}\right)$ ), and $\left(-\lambda_{i}\right)_{i \in I}$ is directed as well by $(* *)$, we may use the Scott-continuity of addition and of multiplication by $a$, and rewrite this as $a \sup _{i \in I}\left(-\lambda_{i}\right)+\sup _{i \in I}\left(-R\left(\nu_{i}\right)\right)=0$, hence to $\inf _{i \in I} \lambda_{i}=0$, considering that $\inf _{i \in I} R\left(\nu_{i}\right)=0$.

Knowing this, and using again that directed suprema are naive suprema, our task consists in showing that $\inf _{i \in I} r_{i}^{\prime}=0$, and that for every $h \in$ 
$\mathcal{L}_{1}^{a}(X, d), \int_{x \in X} h(x) d \nu=\sup _{i \in I}\left(\int_{x \in X} h(x) d \nu_{i}^{\prime}-r_{i}^{\prime}\right)$. The first equality is proved by rewriting $\inf _{i \in I} r_{i}^{\prime} \operatorname{as~inf}_{i \in I} R\left(\nu_{i}\right)+a \inf _{i \in I} \lambda_{i}$, invoking Scott-continuity as above, and then using the equality $\inf _{i \in I} R\left(\nu_{i}\right)=0$ and $(\dagger)$.

For the second equality, $\sup _{i \in I}\left(\int_{x \in X} h(x) d \nu_{i}^{\prime}-r_{i}^{\prime}\right)$ is equal to $\sup _{i \in I}\left(\int_{x \in X} h(x) d \dot{\nu}_{i}+\right.$ $\left.h\left(x_{0}\right) \lambda_{i}-R\left(\nu_{i}\right)-a \lambda_{i}\right)$, which is equal to the $\operatorname{sum}$ of $\sup _{i \in I}\left(\int_{x \in X} h(x) d \stackrel{\circ}{\nu}_{i}-R\left(\nu_{i}\right)\right.$ and of $\left(a-h\left(x_{0}\right)\right) \sup _{i \in I}\left(-\lambda_{i}\right)$, by Scott-continuity of addition and of multiplication by $a-h\left(x_{0}\right)$, again. The first of those summands is equal to $\int_{x \in X} h(x) d \nu$ since $(\nu, 0)$ is the supremum of $\left(\beta\left(\nu_{i}\right)\right)_{i \in I}$ in $\mathbf{B}\left(\mathbf{V}_{\leq 1} X, d_{\mathrm{KR}}^{a}\right)$, and the second one is equal to 0 by $(\dagger)$.

A quasi-metric $d$ on a space $X$ is a-bounded if and only if $d(x, y) \leq a$ for all $x, y \in X$. It is bounded if and only if it is $a$-bounded for some $a \in \mathbb{R}_{+}$.

Remark 6.2. The quasi-metric $d_{K R}^{a}$ is a-bounded on any space of previsions.

Lemma 6.3. If $d$ is an a-bounded quasi-metric on $X$, then $d_{K R}$ and $d_{K R}^{a}$ coincide on $\mathbf{V}_{*} X$, whether $*$ is nothing, " $\leq 1$ ", or " 1 ".

Proof. Clearly, $d_{\mathrm{KR}}^{a} \leq d_{\mathrm{KR}}$. In order to show that $d_{\mathrm{KR}} \leq d_{\mathrm{KR}}^{a}$, let $\nu, \nu^{\prime}$ be any two continuous valuations, and let us consider any $h \in \mathcal{L}_{1}(X, d)$. We claim that there is an $h^{\prime} \in \mathcal{L}_{1}^{a}(X, d)$ such that $\mathrm{d}_{\mathbb{R}}\left(\int_{x \in X} h(x) d \nu, \int_{x \in X} h(x) d \nu^{\prime}\right)=$ $\mathrm{d}_{\mathbb{R}}\left(\int_{x \in X} h^{\prime}(x) d \nu, \int_{x \in X} h^{\prime}(x) d \nu^{\prime}\right)$. This will imply that $\mathrm{d}_{\mathbb{R}}\left(\int_{x \in X} h(x) d \nu, \int_{x \in X} h(x) d \nu^{\prime}\right) \leq$ $d_{\mathrm{KR}}^{a}\left(\nu, \nu^{\prime}\right)$, hence, as $h$ is arbitrary, that $d_{\mathrm{KR}}\left(\nu, \nu^{\prime}\right) \leq d_{\mathrm{KR}}^{a}\left(\nu, \nu^{\prime}\right)$.

For any two points $x, y \in X, \mathrm{~d}_{\mathbb{R}}(h(x), h(y)) \leq d(x, y) \leq a$. This implies that the range of $h$ is included in an interval of length at most $a$, namely an interval $[b, b+a]$ for some $b \in \mathbb{R}_{+}$, or $\{+\infty\}$. In the first case, $h^{\prime}=h-b .1$ fits. In the second case, $\mathbf{d}_{\mathbb{R}}\left(\int_{x \in X} h(x) d \nu, \int_{x \in X} h(x) d \nu^{\prime}\right)=0$ and the constant 0 map fits.

Corollary 6.4. Let $X, d$ be an algebraic complete quasi-metric space with strong basis $\mathcal{B}$, where $d$ is bounded. The space $\mathbf{V}_{1} X, d_{K R}$ is algebraic complete. All the simple probability valuations $\sum_{i=1}^{n} a_{i} \delta_{x_{i}}$ with $x_{i} \in \mathcal{B}$ and $\sum_{i=1}^{n} a_{i}=1$, are center points, and form a strong basis.

Proof. By Lemma $6.3, d_{\mathrm{KR}}=d_{\mathrm{KR}}^{a}$, where $a>0$ is some non-negative real such that $d$ is $a$-bounded. Now apply Theorem 6.1.

Corollary 6.4 is rather restrictive, and we give a better result below. The proof reuses some of the ideas used above.

In a quasi-metric space $X, d$, say that $x \in X$ is an $a$-root if and only if $d(x, y) \leq a$ for every $y \in X$. As a special case, a 0 -root is an element below 
all others in the $\leq d^{+}$ordering. For example, 0 is a 0 -root in $\overline{\mathbb{R}}_{+}$. Every lift $X_{\perp}, d_{\perp}$ of a quasi-metric space $X, d$ is 0-rooted, where $X_{\perp}$ is obtained from $X$ by adding a fresh element $\perp$, and $d_{\perp}$ is defined as $d$ on $X$, and so that $d_{\perp}(\perp, x)=0$ for every $x \in X$. When $X, d$ is standard, the $d_{\perp}$-Scott open subsets of $X_{\perp}$ are exactly $X_{\perp}$ itself, plus all the $d$-Scott open subsets of $X$. In that case, there is a well-known isomorphism between $\mathbf{V}_{1} X_{\perp}$ and $\mathbf{V}_{\leq 1} X$ [9], and we let the reader verify that it extends to an isometry with respect to $d_{\perp \mathrm{KR}}$ and $d_{\mathrm{KR}}$ (resp., $d_{\perp \mathrm{KR}}^{a}$ and $d_{\mathrm{KR}}^{a}$ ). It follows that $\mathbf{V}_{1} X_{\perp}, d_{\perp \mathrm{KR}}$ (resp., $\left.d_{\perp \mathrm{KR}}^{a}\right)$ is algebraic complete for every algebraic complete quasi-metric space $X, d$, as a consequence of Theorem 5.7. Below, we deal with arbitrary rooted, algebraic complete quasi-metric spaces instead of just lifts $X_{\perp}, d_{\perp}$.

We call root any $a$-root, for some $a \in \mathbb{R}_{+}, a>0$. $\mathbb{R}$ has no root.

Lemma 6.5. Let $X, d$ be a standard algebraic quasi-metric space with strong basis $\mathcal{B}$, and $a \in \mathbb{R}_{+}, a>0$. If $X, d$ has an a-root, then, for every $\epsilon>0$, it also has an $(a+\epsilon)$-root in $\mathcal{B}$.

Proof. Assume an $a$-root $x$. By Lemma 5.6, $(x, 0)$ is the supremum of a directed family $\left(x_{i}, r_{i}\right)_{i \in I}$ where each $x_{i}$ is in $\mathcal{B}$. Since $X, d$ is standard, $\inf _{i \in I} r_{i}=0$, so there is an $i \in I$ such that $r_{i}<\epsilon$. We use the fact that $\left(x_{i}, r_{i}\right) \leq d^{+}(x, 0)$ to infer that $d\left(x_{i}, x\right) \leq r_{i}<\epsilon$. It follows that, for every $y \in X, d\left(x_{i}, y\right) \leq d\left(x_{i}, x\right)+d(x, y)<a+\epsilon$.

Theorem 6.6 (Algebraicity for spaces of probabilities, $d_{\mathbf{K R}}$ ). Let $X, d$ be an algebraic complete quasi-metric space, with strong basis $\mathcal{B}$, and with a root $x_{0}$. The space $\mathbf{V}_{1} X, d_{K R}$ is algebraic complete.

All the simple probability valuations $\sum_{i=1}^{n} a_{i} \delta_{x_{i}}$ where $x_{i}$ are center points and $\sum_{i=1}^{n} a_{i}=1$, are center points, and form a strong basis, even when each $x_{i}$ is taken from $\mathcal{B}$.

Proof. By Lemma 6.5, we may assume that $x_{0}$ is also in $\mathcal{B}$. Let now $a \in \mathbb{R}_{+}$, $a>0$, be such that $d\left(x_{0}, x\right) \leq a$ for every $x \in X$.

For all probability valuations $\nu$ and $\nu^{\prime}$ on $X$, for every $h \in \mathcal{L}_{1}(X, d)$, we claim that there is a map $h^{\prime} \in \mathcal{L}_{1}(X, d)$ such that $h^{\prime}\left(x_{0}\right) \leq a$, and such that $\mathrm{d}_{\mathbb{R}}\left(\int_{x \in X} h(x) d \nu, \int_{x \in X} h(x) d \nu^{\prime}\right)=\mathrm{d}_{\mathbb{R}}\left(\int_{x \in X} h^{\prime}(x) d \nu, \int_{x \in X} h^{\prime}(x) d \nu^{\prime}\right)$. The argument is similar to Lemma 6.3. For every $x \in X, h\left(x_{0}\right) \leq h(x)+d\left(x_{0}, x\right)$ since $h$ is 1-Lipschitz, so $h(x) \geq h\left(x_{0}\right)-a$ for every $x \in X$. If $h\left(x_{0}\right)=$ $+\infty$, this implies that $h$ is the constant $+\infty$ map. Then $\int_{x \in X} h(x) d \nu=$ 
$\int_{x \in X} \sup _{k \in \mathbb{N}} k \cdot \mathbf{1}(x) d \nu=\sup _{k \in \mathbb{N}} k=+\infty$, and similarly $\int_{x \in X} h(x) d \nu^{\prime}=+\infty$, so $\mathrm{d}_{\mathbb{R}}\left(\int_{x \in X} h(x) d \nu, \int_{x \in X} h(x) d \nu^{\prime}\right)=0$ : we can take $h^{\prime}=0$. If $h\left(x_{0}\right)<+\infty$, then let $h^{\prime}(x)=h(x)-h\left(x_{0}\right)+a$ for every $x \in X$. Since $h(x) \geq h\left(x_{0}\right)-a$ for every $x \in X, h^{\prime}(x)$ is in $\overline{\mathbb{R}}_{+}$. Clearly $h^{\prime} \in \mathcal{L}_{1}(X, d)$, and $h^{\prime}\left(x_{0}\right)=a$.

Therefore $d_{\mathrm{KR}}\left(\nu, \nu^{\prime}\right)=\sup _{h \in \mathcal{L}_{1}(X, d), h\left(x_{0}\right) \leq a} \mathrm{~d}_{\mathbb{R}}\left(\int_{x \in X} h(x) d \nu, \int_{x \in X} h(x) d \nu^{\prime}\right)$.

The proof is now very similar to Theorem 6.1. Let $\nu \in \mathbf{V}_{1} X$. Then $\eta_{X}[\nu]$ is the supremum of a directed family of simple valuations $\nu_{i}=\sum_{j=1}^{n_{i}} a_{i j} \delta_{\left(x_{i j}, r_{i j}\right)}$, $i \in I$, where each $x_{i j}$ is in $\mathcal{B}$, and $\sum_{j=1}^{n_{i}} a_{i j} \leq 1$. We require $r_{i j}<1$, define $\dot{\nu}_{i}$ as $\sum_{j=1}^{n_{i}} a_{i j} \delta_{x_{i j}}, R\left(\nu_{i}\right)=\sum_{j=1}^{n_{i}} a_{i j} r_{i j}+1-\sum_{j=1}^{n_{i}} a_{i j}$, and $\beta\left(\nu_{i}\right)=\left(\stackrel{\circ}{\nu}_{i}, R\left(\nu_{i}\right)\right)$. Define $i \preceq i^{\prime}$ if and only if $\nu_{i} \leq \nu_{i^{\prime}}$, for all $i, i^{\prime} \in I$. We have again: $(*)$ for all $i \preceq i^{\prime}$ in $I, \beta\left(\nu_{i}\right) \leq_{\mathrm{KR}_{\mathrm{R}}^{+}}^{+} \beta\left(\nu_{i^{\prime}}\right)$.

Now define $\nu_{i}^{\prime}$ as $\stackrel{\circ}{\nu}_{i}+\lambda_{i} \delta_{x_{0}}$, and $r_{i}^{\prime}=R\left(\nu_{i}\right)+a \lambda_{i}$, where $\lambda_{i}=1-\stackrel{\circ}{\nu}_{i}(X)$, exactly as in the proof of Theorem 6.1. We show, as before: $(* *)$ for all $i \preceq i^{\prime}$ in $I, \lambda_{i} \geq \lambda_{i^{\prime}} ;(* * *)$ for all $i \preceq i^{\prime}$ in $I, r_{i}^{\prime} \geq r_{i^{\prime}}^{\prime}$.

Let now $h$ be an arbitrary element from $\mathcal{L}_{1}(X, d)$ such that $h\left(x_{0}\right) \leq a$, and assume $i \preceq i^{\prime}$. We have the following chain of inequalities - exactly the same as in Theorem 6.1:

$$
\begin{aligned}
& \mathrm{d}_{\mathbb{R}}\left(\int_{x \in X} h(x) d \nu_{i}^{\prime}, \int_{x \in X} h(x) d \nu_{i^{\prime}}^{\prime}\right) \\
& =\max \left(\int_{x \in X} h(x) d \stackrel{\circ}{i}_{i}+h\left(x_{0}\right) \lambda_{i}-\int_{x \in X} h(x) d{\stackrel{\circ}{i^{\prime}}}^{\prime}-h\left(x_{0}\right) \lambda_{i^{\prime}}, 0\right) \\
& \leq \max \left(R\left(\nu_{i}\right)-R\left(\nu_{i^{\prime}}\right)+h\left(x_{0}\right)\left(\lambda_{i}-\lambda_{i^{\prime}}\right), 0\right) \\
& \quad \text { since } d_{\mathrm{KR}}\left(\stackrel{\circ}{\nu}_{i},{\stackrel{\nu}{i^{\prime}}}^{\prime}\right) R\left(\nu_{i}\right)-R\left(\nu_{i^{\prime}}\right), \operatorname{using}(*) \\
& \leq \max \left(R\left(\nu_{i}\right)-R\left(\nu_{i^{\prime}}\right)+a\left(\lambda_{i}-\lambda_{i^{\prime}}\right), 0\right) \\
& \quad \operatorname{since} h\left(x_{0}\right) \leq a, \text { and } \lambda_{i} \geq \lambda_{i^{\prime}} \text { by }(* *) \\
& =\max \left(r_{i}^{\prime}-r_{i^{\prime}}^{\prime}, 0\right)=r_{i}^{\prime}-r_{i^{\prime}}^{\prime} \quad \text { by }(* * *) .
\end{aligned}
$$

This shows that for all $i \preceq i^{\prime}$ in $I, d_{\mathrm{KR}}\left(\nu_{i}^{\prime}, \nu_{i^{\prime}}^{\prime}\right) \leq r_{i}^{\prime}-r_{i^{\prime}}^{\prime}$, since we have taken the precaution to show that $d_{\mathrm{KR}}\left(\nu_{i}^{\prime}, \nu_{i^{\prime}}^{\prime}\right)$ is the supremum of $\mathrm{d}_{\mathbb{R}}\left(\int_{x \in X} h(x) d \nu_{i}^{\prime}\right.$, $\left.\int_{x \in X} h(x) d \nu_{i^{\prime}}^{\prime}\right)$ over all $h \in \mathcal{L}_{1}(X, d)$ such that $h\left(x_{0}\right) \leq a$. Therefore $\left(\nu_{i}^{\prime}, r_{i}^{\prime}\right) \leq d_{\mathrm{KR}}^{+}$ $\left(\nu_{i^{\prime}}^{\prime}, r_{i^{\prime}}^{\prime}\right)$ for all $i \preceq i^{\prime}$ in $I$. In particular, $\left(\nu_{i}^{\prime}, r_{i}^{\prime}\right)_{i \in I}$ is directed.

The proof that $\left(\nu_{i}^{\prime}, r_{i}^{\prime}\right) \leq d_{\mathrm{KR}}^{+}(\nu, 0)$ is also as in the proof of Theorem 6.1. It suffices to show that for every $h \in \mathcal{L}_{1}(X, d)$ with $h\left(x_{0}\right) \leq a, \int_{x \in X} h(x) d \dot{\nu}_{i}+$ $h\left(x_{0}\right) \lambda_{i} \leq \int_{x \in X} h(x) d \nu+R\left(\nu_{i}\right)+a \lambda_{i}$. We know that $\int_{x \in X} h(x) d \dot{\nu}_{i} \leq \int_{x \in X} h(x) d \nu+$ $R\left(\nu_{i}\right)$ since $\beta\left(\nu_{i}\right) \leq d_{\mathrm{KR}}^{a+}(\nu, 0)$, and we conclude since $h\left(x_{0}\right) \leq a$. 
Finally, we show that $(\nu, 0)$ is also the supremum of the directed family $\left(\nu_{i}^{\prime}, r_{i}^{\prime}\right)_{i \in I}$ in $\mathbf{B}\left(\mathbf{V}_{1} X, d_{\mathrm{KR}}\right)$ by first showing: $(\dagger) \inf _{i \in I} \lambda_{i}=0$; then by showing that it is the naive supremum of the family, that is, by showing that $\inf _{i \in I} r_{i}^{\prime}=$ 0 and that for every $h \in \mathcal{L}_{1}(X, d), \int_{x \in X} h(x) d \nu=\sup _{i \in I}\left(\int_{x \in X} h(x) d \nu_{i}^{\prime}-\right.$ $\left.r_{i}^{\prime}\right)$. The first equality is easy, considering $(\dagger)$. For the second equality, $\sup _{i \in I}\left(\int_{x \in X} h(x) d \nu_{i}^{\prime}-r_{i}^{\prime}\right)$ is equal to $\sup _{i \in I}\left(\int_{x \in X} h(x) d \nu_{i}+h\left(x_{0}\right) \lambda_{i}-R\left(\nu_{i}\right)-\right.$ $\left.a \lambda_{i}\right)$, which is equal to the $\operatorname{sum}$ of $\sup _{i \in I}\left(\int_{x \in X} h(x) d \dot{\nu}_{i}-R\left(\nu_{i}\right)\right.$ and of $(a-$ $\left.h\left(x_{0}\right)\right) \sup _{i \in I}\left(-\lambda_{i}\right)$, by Scott-continuity of addition and of multiplication by $a-h\left(x_{0}\right)$, again. (We use $h\left(x_{0}\right) \leq a$ again here.) The first of those summands is equal to $\int_{x \in X} h(x) d \nu$ since $(\nu, 0)$ is the (naive) supremum of $\left(\beta\left(\nu_{i}\right)\right)_{i \in I}$ in $\mathbf{B}\left(\mathbf{V}_{\leq 1} X, d_{\mathrm{KR}}^{a}\right)$, and the second one is equal to 0 by $(\dagger)$.

Remark 6.7. Under the assumptions of Theorem 6.6, all simple probability valuations supported on center points are themselves center points of $\mathbf{V}_{1} X$. The converse is certainly not true: we will see in Section 7 that $d_{K R}$ is a metric if $d$ is a metric; in that case, every element of $\mathbf{V}_{1} X$ is a center point.

\subsection{Further remarks}

We can refine Lemma 5.1 as follows. Under the same assumptions, in particular assuming that each $x_{i}$ is a center point, the sum $\sum_{i=1}^{n} a_{i} h\left(x_{i}\right)$ is just the integral of $h$ with respect to the simple valuation $\sum_{i=1}^{n} a_{i} \delta_{x_{i}}$. Since all continuous maps from a compact space to $\left(\overline{\mathbb{R}}_{+}\right)^{\mathrm{d}}$ reach their maximum, Lemma 5.1 immediately entails:

Lemma 6.8. Let $X, d$ be a continuous quasi-metric space, let $\nu=\sum_{i=1}^{n} a_{i} \delta_{x_{i}}$ be a simple valuation on $X$ such that every $x_{i}$ is a center point, and let $\nu^{\prime}$ be a continuous valuation on $X$. There is an $h \in \mathcal{L}_{1}(X, d)$ such that $\left.d_{K R}\left(\nu, \nu^{\prime}\right)=\mathrm{d}_{\mathbb{R}}\left(\sum_{i=1}^{n} a_{i} h\left(x_{i}\right), \int_{x \in X} h(x) d \nu^{\prime}\right)\right)$; in other words, the supremum in (1) is reached.

Similarly, for every $a>0$, the supremum in (3) is reached: there is an $h \in \mathcal{L}_{1}^{a}(X, d)$ such that $d_{K R}^{a}\left(\nu, \nu^{\prime}\right)=\mathrm{d}_{\mathbb{R}}\left(\sum_{i=1}^{n} a_{i} h\left(x_{i}\right), \int_{x \in X} h(x) d \nu^{\prime}\right)$.

Out of curiosity, we realize that one can make such an $h$ explicit. This will come in handy later.

Definition $6.9(x \nearrow b)$. Let $X, d$ be a quasi-metric space. For each center point $x \in X$ and each $b \in \overline{\mathbb{R}}_{+}$, let $x \nearrow b: X \rightarrow \overline{\mathbb{R}}_{+}$map every $y \in X$ to the smallest element $t \in \overline{\mathbb{R}}_{+}$such that $b \leq t+d(x, y)$. 
When $b \neq+\infty$, we might have said, more simply: $(x \nearrow b)(y)=\max (b-$ $d(x, y), 0)$. The definition caters for the general situation. When $b=+\infty$, $(x \nearrow+\infty)(y)$ is equal to 0 if $d(x, y)=+\infty$, and to $+\infty$ if $d(x, y)<+\infty$.

Lemma 6.10. Let $X, d$ be a standard quasi-metric space, $x$ be a center point of $X, d$ and $b \in \overline{\mathbb{R}}_{+}$. The function $x \nearrow b$ is 1-Lipschitz continuous.

Proof. It is clear that $x \nearrow b$ is 1-Lipschitz. Let us show that it is continuous. Let $f=x \nearrow b$, and $f^{\prime}(y, r)=f(y)-r$. For every $s \in \mathbb{R}$, we wish to show that $\left.\left.f^{\prime-1}(] s,+\infty\right]\right)$ is Scott-open.

If $b \neq+\infty$, a formal ball $(y, r)$ is in $\left.\left.f^{\prime-1}(] s,+\infty\right]\right)$ if and only if $\max (b-$ $d(x, y), 0)-r>s$. This is equivalent to $d(x, y)+r<b-s$ or $r<-s$. The set $V_{-s}=\{(y, r) \mid r<-s\}$ is Scott-open since $X, d$ is standard. The condition $d(x, y)+r<b-s$ is vacuously false if $b \leq s$, and is equivalent to $d^{+}((x, 0),(y, r))<b-s$ otherwise. It follows that $\left.\left.f^{\prime-1}(] s,+\infty\right]\right)$ is equal to $V_{-s}$ if $b \leq s$, or to $V_{-s} \cup B_{(x, 0),<b-s}^{d^{+}}$otherwise. This is Scott-open in any case, because $x$ is a center point.

If $b=+\infty$, then $\left.\left.(y, r) \in f^{\prime-1}(] s,+\infty\right]\right)$ entails that $-r>s$ if $d(x, y)=$ $+\infty$, or $d(x, y)<+\infty$. Conversely, if $-r>s$, then $f^{\prime}(y, r)>s$, whether $d(x, y)=+\infty$ or $d(x, y)<+\infty$, and if $d(x, y)<+\infty$, then $f^{\prime}(y, r)=+\infty>$ s. Also, $d(x, y)<+\infty$ if and only if $d^{+}((x, 0),(y, r))<+\infty$, if and only if $d^{+}((x, 0),(y, r))<N$ for some natural number $N$. Therefore $\left.\left.f^{\prime-1}(] s,+\infty\right]\right)$ is equal to $V_{-s} \cup \bigcup_{N} B_{(x, 0),<N}^{d^{+}}$, which is Scott-open.

Lemma 6.11. Let $X, d$ be a standard quasi-metric space, $x_{i}$ be center points of $X, d$ and $b_{i} \in \overline{\mathbb{R}}_{+}, 1 \leq i \leq n$. The function $\bigvee_{i=1}^{n} x_{i} \nearrow b_{i}$, which maps every $y \in X$ to $\max \left\{\left(x_{i} \nearrow b_{i}\right)(y) \mid 1 \leq i \leq n\right\}$, is the smallest 1-Lipschitz map $f$ (hence also the smallest function in $\mathcal{L}_{1}(X, d)$ ) such that $f\left(x_{i}\right) \geq b_{i}$ for every $i, 1 \leq i \leq n$.

Proof. First, $f=\bigvee_{i=1}^{n} x_{i} \nearrow b_{i}$ is in $\mathcal{L}_{1}(X, d)$, by Lemma 6.10 and the fact that the pointwise max of two $\alpha$-Lipschitz continuous maps is $\alpha$-Lipschitz continuous. For any other 1-Lipschitz map $h$ such that $h\left(x_{i}\right) \geq b_{i}, 1 \leq i \leq n$, for every $y \in X, h\left(x_{i}\right) \leq h(y)+d\left(x_{i}, y\right)$. In other words, $h(y)$ is a number $t \in \overline{\mathbb{R}}_{+}$such that $b_{i} \leq t+d\left(x_{i}, y\right)$. The smallest such $t$ is $x_{i} \nearrow b_{i}(y)$ by definition, so $h(y) \geq\left(x_{i} \nearrow b_{i}\right)(y)$. Since that holds for every $i$ and every $y$, $h \geq f$. 
Proposition 6.12. Let $X, d$ be a continuous complete quasi-metric space, let $\nu=\sum_{i=1}^{n} a_{i} \delta_{x_{i}}$ be a simple valuation on $X$ such that every $x_{i}$ is a center point, and let $\nu^{\prime}$ be a continuous valuation on $X$. There are numbers $b_{i} \in \overline{\mathbb{R}}_{+}$, $1 \leq i \leq n$, such that $d_{K R}\left(\nu, \nu^{\prime}\right)=\mathrm{d}_{\mathbb{R}}\left(\sum_{i=1}^{n} a_{i} h\left(x_{i}\right), \int_{x \in X} h(x) d \nu^{\prime}\right)$, where $h=\bigvee_{i=1}^{n} x_{i} \nearrow b_{i}$.

Similarly, for every $a \in \mathbb{R}_{+}, a>0$, there are numbers $b_{i} \in[0, \alpha a]$, $1 \leq i \leq n$, such that $d_{K R}^{a}\left(\nu, \nu^{\prime}\right)=\mathrm{d}_{\mathbb{R}}\left(\sum_{i=1}^{n} a_{i} h\left(x_{i}\right), \int_{x \in X} h(x) d \nu^{\prime}\right)$, where $h=\bigvee_{i=1}^{n} x_{i} \nearrow b_{i}$.

Proof. We deal with the $d_{\mathrm{KR}}$ case, the $d_{\mathrm{KR}}^{a}$ case is similar.

Using Lemma 6.8, there is an $h_{0} \in \mathcal{L}_{1}(X, d)$ such that $d_{\mathrm{KR}}\left(\nu, \nu^{\prime}\right)=$ $\mathrm{d}_{\mathbb{R}}\left(\sum_{i=1}^{n} a_{i} h_{0}\left(x_{i}\right), \int_{x \in X} h_{0}(x) d \nu^{\prime}\right)$. Let $b_{i}=h_{0}\left(x_{i}\right), 1 \leq i \leq n$, and define $h$ as $\bigvee_{i=1}^{n} x_{i} \nearrow b_{i}$. By Lemma $6.11, h$ is in $\mathcal{L}_{1}(X, d)$ and $h \leq h_{0}$, so $\int_{x \in X} h(x) d \nu^{\prime} \leq \int_{x \in X} h_{0}(x) d \nu^{\prime}$. Since $\mathrm{d}_{\mathbb{R}}$ is antitone in its second argument, $\left.d_{\mathrm{KR}}\left(\nu, \nu^{\prime}\right) \leq \mathrm{d}_{\mathbb{R}}\left(\sum_{i=1}^{n} a_{i} h_{0}\left(x_{i}\right), \int_{x \in X} h(x) d \nu^{\prime}\right)\right)$, and since $h_{0}\left(x_{i}\right)=h\left(x_{i}\right)$ for each $i, d_{\mathrm{KR}}\left(\nu, \nu^{\prime}\right) \leq \mathrm{d}_{\mathbb{R}}\left(\sum_{i=1}^{n} a_{i} h\left(x_{i}\right), \int_{x \in X} h(x) d \nu^{\prime}\right)$. The reverse inequality is by definition of $d_{\mathrm{KR}}$.

\section{Probabilities on metric spaces}

Among all quasi-metric spaces, the case of metric spaces stands out.

Lemma 7.1. Let $X, d$ be a metric space. Then $d_{K R}$ is a metric, not just a quasi-metric, on $\mathbf{V}_{1} X$. The same holds for $d_{K R}^{a}$, for every $a \in \mathbb{R}_{+}, a>0$.

Proof. As a matter of simplification, recall that on a metric space $X, d$, the $d$-Scott topology coincides with the open ball topology. As a result, every 1-Lipschitz map is automatically continuous, hence 1-Lipschitz continuous since every metric space is standard.

We start with the case of $d_{\mathrm{KR}}^{a}$. For every $h \in \mathcal{L}_{1}^{a}(X, d), a-h$ is 1-Lipschitz: for all $x, y \in X,(a-h(x))-(a-h(y))=h(y)-h(x) \leq d(y, x)=d(x, y)$, using the fact that $h$ is 1-Lipschitz and that $d$ is a metric. Moreover, $a-h$ is bounded from above by $a$, so $a-h$ is in $\mathcal{L}_{1}^{a}(X, d)$.

For every subprobability valuation $\nu$ on $X, \int_{x \in X}(a-h)(x) d \nu=a-$ $\int_{x \in X} h(x) d \nu$. Indeed, by linearity $\int_{x \in X}(a-h)(x) d \nu+\int_{x \in X} h(x) d \nu=\int_{x \in X} a d \nu=$ $a$.

For all probability valuations $\nu, \nu^{\prime}$ on $X, \mathrm{~d}_{\mathbb{R}}\left(\int_{x \in X}(a-h)(x) d \nu^{\prime}, \int_{x \in X}(a-\right.$ $h)(x) d \nu)$ is therefore equal to $\mathrm{d}_{\mathbb{R}}\left(a-\int_{x \in X} h(x) d \nu^{\prime}, a-\int_{x \in X} h(x) d \nu\right)$, hence to $\mathrm{d}_{\mathbb{R}}\left(\int_{x \in X} h(x) d \nu, \int_{x \in X} h(x) d \nu^{\prime}\right)$. It follows that for every $h \in \mathcal{L}_{1}^{a}(X, d)$, there is 
an $h^{\prime} \in \mathcal{L}_{1}^{a}(X, d)$, namely $h^{\prime}=a-h$, such that $\mathrm{d}_{\mathbb{R}}\left(\int_{x \in X} h^{\prime}(x) d \nu^{\prime}, \int_{x \in X} h^{\prime}(x) d \nu\right)=$ $\mathrm{d}_{\mathbb{R}}\left(\int_{x \in X} h(x) d \nu, \int_{x \in X} h(x) d \nu^{\prime}\right)$. Therefore $d_{\mathrm{KR}}^{a}\left(\nu, \nu^{\prime}\right) \leq d_{\mathrm{KR}}^{a}\left(\nu^{\prime}, \nu\right)$. By symmetry, we conclude that $d_{\mathrm{KR}}^{a}\left(\nu, \nu^{\prime}\right)=d_{\mathrm{KR}}^{a}\left(\nu^{\prime}, \nu\right): d_{\mathrm{KR}}^{a}$ is a metric.

To show that $d_{\mathrm{KR}}$ is a metric, it is enough to observe that $d_{\mathrm{KR}}\left(\nu, \nu^{\prime}\right)$ is equal to $\sup _{a \in \mathbb{R}_{+}, a>0} d_{\mathrm{KR}}^{a}\left(\nu, \nu^{\prime}\right)$ (Lemma 3.9), and to use the fact that $d_{\mathrm{KR}}^{a}$ is a metric.

Since $d_{\mathrm{KR}}\left(\nu, \nu^{\prime}\right)=d_{\mathrm{KR}}\left(\nu^{\prime}, \nu\right)$ in the metric case, $d_{\mathrm{KR}}\left(\nu, \nu^{\prime}\right)$ is also equal to $\max \left(d_{\mathrm{KR}}\left(\nu, \nu^{\prime}\right), d_{\mathrm{KR}}\left(\nu^{\prime}, \nu\right)\right)$, which is easily seen to be equal to $\sup _{h \in \mathcal{L}_{1}(X, d)} \max \left(\mathrm{d}_{\mathbb{R}}\left(\int_{x \in X} h(x) d \nu\right.\right.$, $\left.\left.\int_{x \in X} h(x) d \nu^{\prime}\right), \mathbf{d}_{\mathbb{R}}\left(\int_{x \in X} h(x) d \nu^{\prime}, \int_{x \in X} h(x) d \nu\right)\right)$. The inner maximum is the symmetrized distance $\mathrm{d}_{\mathbb{R}}^{\text {sym }}$, defined by $\mathrm{d}_{\mathbb{R}}^{\text {sym }}(a, b)=|a-b|$ for all $a, b \in \mathbb{R}_{+}$. Therefore, we obtain the formula:

$$
\begin{aligned}
d_{\mathrm{KR}}\left(\nu, \nu^{\prime}\right) & =\sup _{h 1 \text {-Lipschitz }}\left|\int_{x \in X} h(x) d \nu-\int_{x \in X} h(x) d \nu^{\prime}\right| \\
& =\sup _{h \text { 1-Lipschitz bounded }}\left|\int_{x \in X} h(x) d \nu-\int_{x \in X} h(x) d \nu^{\prime}\right|,
\end{aligned}
$$

for $\nu, \nu^{\prime} \in \mathbf{V}_{1} X$, in the case where $d$ is a metric on $X$. (The second equality is by Lemma 3.8.)

Similarly,

$$
d_{\mathrm{KR}}^{a}\left(\nu, \nu^{\prime}\right)=\sup _{h \text { 1-Lipschitz bounded by } a}\left|\int_{x \in X} h(x) d \nu-\int_{x \in X} h(x) d \nu^{\prime}\right|,
$$

for $\nu, \nu^{\prime} \in \mathbf{V}_{1} X$, assuming again that $d$ is a metric. We recognize the usual formula for the Kantorovich-Rubinstein metric when $a=1$.

Theorem 4.15 then states:

Theorem 7.2. For every complete metric space $X, d$, the space $\mathbf{V}_{1} X$ with the Kantorovich-Rubinstein metric (12), or with the a-bounded KantorovichRubinstein metric (13), $a \in \mathbb{R}_{+}, a>0$, is a complete metric space.

We haven't cared to mention that the simple normalized valuations $\sum_{i=1}^{n} a_{i} \delta_{x_{i}}$ with $x_{i}$ center points in $X, d, \sum_{i=1}^{n} a_{i}=1$, are center points. This is trivially true, because every point of a metric space is a center point. In fact, in the case of Theorem 7.2, every probability valuation is a center point, and every complete metric space is algebraic complete.

Theorem 7.2 resembles the classical result that, if $X, d$ is a complete separable metric space, then $\mathbf{V}_{1} X, d_{\mathrm{KR}}$ is a complete (separable) metric space. Note that we do not need $X$ to be separable for Theorem 7.2 to hold. 


\section{Continuity}

The case of continuous complete quasi-metric spaces follows from the algebraic case, because the continuous complete quasi-metric spaces are exactly the 1-Lipschitz continuous retracts of algebraic complete quasi-metric spaces [20, Theorem 7.9]. A 1-Lipschitz continuous retraction of $Y, \partial$ onto $X, d$ is a pair of 1-Lipschitz continuous maps $r: Y, \partial \rightarrow X, d$ and $s: X, d \rightarrow Y, \partial$ such that $r \circ s=\operatorname{id}_{X}$. We also say that $r$ itself is the retraction, that $X, d$ is the retract, and that $s$ is the section.

Recall the map $\mathbf{V} f$ from Lemma 4.8.

Lemma 8.1. Let $X, d$ and $Y, \partial$ be two continuous complete quasi-metric spaces, and $f: X, d \mapsto Y, \partial$ be a 1-Lipschitz continuous map. The restriction of $\mathbf{V f}$ to $\mathbf{V}_{\leq 1} X$ is a 1-Lipschitz continuous map from $\mathbf{V}_{\leq 1} X, d_{K R}$ to $\mathbf{V}_{\leq 1} Y, \partial_{K R}$, and also from $\mathbf{V}_{\leq 1} X, d_{K R}^{a}$ to $\mathbf{V}_{\leq 1} Y, \partial_{K R}^{a}$ for every $a \in \mathbb{R}_{+}, a>0$.

Similarly with $\mathbf{V}_{1}$ instead of $\mathbf{V}_{\leq 1}$.

Proof. Lemma 4.8 says that $\mathbf{V} f$ is 1 -Lipschitz, so $\mathbf{B}^{1}(\mathbf{V} f)$ is monotonic. The same lemma shows that $\mathbf{V} f$ maps subprobability valuations (i.e., elements of $\left.\mathbf{V}_{\leq 1} X\right)$ to subprobability valuations, and probability valuations (i.e., elements of $\left.\mathbf{V}_{1} X\right)$ to probability valuations.

By Theorem 4.15, $\mathbf{V}_{\leq 1} X, d_{\mathrm{KR}}$ and $\mathbf{V}_{\leq 1} Y, \partial_{\mathrm{KR}}$ are complete, and directed suprema in their spaces of formal balls are naive suprema. Similarly with $d_{\mathrm{KR}}^{a}$ and $\partial_{\mathrm{KR}}^{a}$ in lieu of $d_{\mathrm{KR}}$ and $\partial_{\mathrm{KR}}$, or with $\mathbf{V}_{1}$ instead of $\mathbf{V}_{\leq 1}$. By Lemma 4.9, $\mathbf{B}^{1}(\mathbf{V} f)$ preserves naive suprema, hence all directed suprema. It must therefore be Scott-continuous, which shows the claim.

Let $X, d$ be a continuous complete quasi-metric space. There is an algebraic complete quasi-metric space $Y, \partial$ and there are two 1-Lipschitz continuous maps $r: Y, \partial \rightarrow X, d$ and $s: X, d \rightarrow Y, \partial$ such that $r \circ s=\mathrm{id}_{X}$.

By Lemma 8.1, $\mathbf{V r}$ and $\mathbf{V} s$ are also 1-Lipschitz continuous, and clearly $\mathbf{V} r \circ \mathbf{V} s=\operatorname{id}_{\mathbf{V}_{\leq 1} X}$, so $\mathbf{V}_{\leq 1} X, d_{\mathrm{KR}}$ is a 1-Lipschitz continuous retract of $\mathbf{V}_{\leq 1} Y, \partial_{\mathrm{KR}}$. (Similarly with $d_{\mathrm{KR}}^{a}$ and $\partial_{\mathrm{KR}}^{a}$.) Theorem 5.7 states that $\mathbf{V}_{\leq 1} Y, \partial_{\mathrm{KR}}$ (resp., $\partial_{\mathrm{KR}}^{a}$ ) is algebraic complete, whence:

Theorem 8.2 (Continuity for spaces of subprobabilities). Let $X, d$ be a continuous complete quasi-metric space. The quasi-metric spaces $\mathbf{V}_{\leq 1} X, d_{K R}$ and $\mathbf{V}_{\leq 1} X, d_{K R}^{a}\left(a \in \mathbb{R}_{+}, a>0\right)$ are continuous complete.

Together with Lemma 8.1, and Theorem 5.7 for the algebraic case, we obtain. 
Corollary 8.3. $\mathbf{V}_{\leq 1}, d_{K R}$ defines an endofunctor on the category of continuous complete quasi-metric spaces and 1-Lipschitz continuous map. Similarly with $d_{K R}^{a}$ instead of $d_{K R}(a>0)$, or with algebraic instead of continuous.

Remark 8.4. Let $X$ be a poset. We recall from Remark 5.8 that $X$ is a continuous dcpo if and only if $X, d_{\leq}$is a continuous complete quasi-metric space. Remark 3.3 tells us that $d_{K R}$ is the $\{0,+\infty\}$-valued quasi-metric associated with the stochastic ordering on $\mathbf{V}_{\leq 1} X$, so Theorem 8.2 specializes to the following celebrated result by Jones [21, 22] that $\mathbf{V}_{\leq 1} X$ is a continuous dcpo for every continuous dcpo $X$.

Using Remark 3.6 instead, we obtain that, for a continuous dcpo $X$, $\mathbf{V}_{\leq 1} X$ is a continuous complete quasi-metric space in Sünderhauf's sup quasimetric (namely, $d_{K R}^{1}$ ).

Using Theorem 6.1, the same argument produces:

Theorem 8.5 (Continuity for spaces of probabilities, $d_{\mathbf{K R}}^{a}$ ). Let $X, d$ be a continuous complete quasi-metric space. The quasi-metric space $\mathbf{V}_{1} X, d_{K R}^{a}$ is continuous complete for every $a \in \mathbb{R}_{+}, a>0$.

Together with Lemma 8.1, and Theorem 6.1 for the algebraic case, we obtain:

Corollary 8.6. $\mathbf{V}_{1}, d_{K R}^{a}$ defines an endofunctor on the category of continuous (resp., algebraic) complete quasi-metric spaces and 1-Lipschitz continuous map.

Remark 8.7. As in Remark 8.4, we obtain that for every continuous dcpo $X, \mathbf{V}_{1} X$ is a continuous complete quasi-metric space in Sünderhauf's sup quasi-metric $d_{K R}^{1}$.

In the case of $d_{\mathrm{KR}}$, and rooted quasi-metric spaces, the same argument works again, but we have to show that if $X, d$ is not just continuous complete but also rooted, then the algebraic complete quasi-metric space $Y, \partial$ of which $X, d$ is a 1-Lipschitz continuous retract, as guaranteed by Theorem 7.9 of $[20]$, is also rooted.

To this end, we need to know that $Y, \partial$ is the so-called formal ball completion of $X, d$, defined as follows (see Section 7 of [20]). Let $(x, r) \prec(y, s)$ if and only if $d(x, y)<r-s$. A rounded ideal of formal balls on $X, d$ is a set $D \subseteq \mathbf{B}(X, d)$ that is $\prec$-directed in the sense that every finite subset of $D$ is $\prec$-below some element of $D$, and $\prec$-downwards-closed, in the 
sense that any element $\prec$-below some element of $D$ is in $D$. The aperture of such a set $D$ is $\alpha(D)=\inf \{r \mid(x, r) \in D\}$. The elements of $Y$ are exactly the rounded ideals in this sense that have aperture 0 . The quasi-metric $\partial$ is defined by $\partial\left(D, D^{\prime}\right)=\sup _{b \in D} \inf _{b^{\prime} \in D^{\prime}} d^{+}\left(b, b^{\prime}\right)$. The set $\Downarrow(x, 0)=\{(y, r) \in \mathbf{B}(X, d) \mid(y, r) \prec(x, 0)\}$ is an element of $Y$ for every $x \in X$. The section maps every $x \in X$ to $\Downarrow(x, 0)$, and the retraction $d$-lim maps every rounded ideal $D$ such that $\alpha(D)=0$ to the unique point $x \in X$ such that $\sup D=(x, 0)$. We need to know that to show:

Lemma 8.8. The formal ball completion of a quasi-metric space $X, d$ with an a-root $x\left(a \in \mathbb{R}_{+}, a>0\right)$ has an a-root, namely $\Downarrow(x, 0)$.

Proof. Let $D$ be an element of the formal ball completion. We must show that $\partial(\Downarrow(x, 0), D) \leq a$, where $\partial$ is defined as above. For every $b=(z, t) \in$ $\Downarrow(x, 0)$, by definition $d(z, x)<t$. For every $b^{\prime}=(y, s) \in D, d^{+}\left(b, b^{\prime}\right)=$ $\max (d(z, y)-t+s, 0) \leq \max (d(z, x)+d(x, y)-t+s, 0) \leq \max (d(x, y)+s, 0)$. Since $x$ is an $a$-root, this is less than or equal to $a+s$. We use the fact that $\alpha(D)=0$ to make $s$ arbitrarily small. Therefore $\inf _{b^{\prime} \in D} d^{+}\left(b, b^{\prime}\right) \leq a$. Taking suprema over $b \in \Downarrow(x, 0)$, we obtain $\partial(\Downarrow(x, 0), D) \leq a$.

Since $Y, \partial$ is rooted, we can now use Theorem 6.6 and the same retractionbased argument to show:

Theorem 8.9 (Continuity for spaces of probabilities, $d_{\mathrm{KR}}$, rooted case). Let $X, d$ be a continuous complete quasi-metric space with an a-root $x$. The quasi-metric space $\mathbf{V}_{1} X, d_{K R}$ is continuous complete, and $\delta_{x}$ is an a-root.

Proof. Only the fact that $\delta_{x}$ is an $a$-root remains to be proved. Let $\nu \in$ $\mathbf{V}_{1} X$, and $h \in \mathcal{L}_{1} X$. Then:

$$
\begin{aligned}
h(x) & =\int_{y \in X} h(x) d \nu \quad \text { since } \nu(X)=1 \\
& \leq \int_{y \in X} h(y)+d(x, y) d \nu \quad \text { since } h \text { is 1-Lipschitz } \\
& \leq \int_{y \in X} h(y) d \nu+a,
\end{aligned}
$$

since $d(x, y) \leq a$ for every $a \in X$, and $\nu(X)=1$. Hence $\mathrm{d}_{\mathbb{R}}\left(\int_{y \in X} h(y) d \delta_{x}, \int_{y \in x} h(y) d \nu\right) \leq$ a. Taking suprema over $h$, we obtain $d_{\mathrm{KR}}\left(\delta_{x}, \nu\right) \leq a$. 
Corollary 8.10. $\mathbf{V}_{1}, d_{K R}$ defines an endofunctor on the category of continuous (resp., algebraic) complete quasi-metric spaces with a root and 1-Lipschitz continuous map.

Remark 8.11. For every quasi-metric space $X, d, \mathbf{V}_{\leq 1} X, d_{K R}$ and $\mathbf{V} X, d_{K R}$ have a root, namely the zero valuation. That holds even when $X, d$ does not have a root. Indeed, $0 \leq \nu$ for every continuous valuation $\nu$, namely $d(0, \nu)=0$.

We finish by studying bases of $\mathbf{B}\left(\mathbf{V}_{*} X, d_{\mathrm{KR}}\right)$ and $\mathbf{B}\left(\mathbf{V}_{*} X, d_{\mathrm{KR}}^{a}\right), a>0$, where $*$ is " $\leq 1$ " or " 1 ". In the various cases that we studied where $X, d$ is algebraic complete (Theorems 5.7, 6.1, 6.6), we have a basis of simple (sub)probability valuations $\xi=\sum_{i=1}^{n} a_{i} \delta_{x_{i}}$, where each $x_{i}$ is a center point in $X, d$. In the continuous case, we have:

Proposition 8.12. Let $X, d$ be a continuous complete quasi-metric space. Let also * denote either " $\leq 1$ " or " 1 ". A basis of the continuous dcpo $\mathbf{B}\left(\mathbf{V}_{*} X, d_{K R}\right)$ (assuming $X, d$ rooted if $*$ is " 1 "; resp., $\mathbf{B}\left(\mathbf{V}_{\leq 1} X, d_{K R}^{a}\right), a>0$ ) is given by the formal balls $(\xi, t)$ where $\xi$ is a simple (sub)probability valuation.

Proof. In each case, $\mathbf{V}_{*} X, d_{\mathrm{KR}}$ (resp., $d_{\mathrm{KR}}^{a}$ ) is a 1-Lipschitz continuous retract of $\mathbf{V}_{*} Y, \partial_{\mathrm{KR}}$ (resp., $\partial_{\mathrm{KR}}^{a}$ ) where $Y, \partial$ is algebraic complete, with retraction $\mathbf{V} r$ and section $\mathbf{V} s$. Then $\mathbf{B}^{1}(\mathbf{V} r)$ and $\mathbf{B}^{1}(\mathbf{V} s)$ form a retraction-section pair in the category of dcpos and Scott-continuous map. A retract of a continuous dcpo is also a continuous dcpo, and a basis of the retract is given by the image of any given basis by the retraction (see Exercise 5.1.44 of [16], for example). Hence a basis is given by the simple (sub)probability valuations of the form $\mathbf{B}^{1}(\mathbf{V} r)\left(\sum_{i=1}^{n} a_{i} \delta_{y_{i}}, t\right)$, where each $y_{i}$ is a center point and $t \in \mathbb{R}_{+}-$ alternatively, of the form $\left(\sum_{i=1}^{n} a_{i} \delta_{r\left(y_{i}\right)}, t\right)$. In particular, those are all formal balls $(\xi, r)$ where $\xi$ is a simple (sub)probability valuation. It follows that every formal ball on $\mathbf{V}_{*} X, d_{\mathrm{KR}}$ (resp., $d_{\mathrm{KR}}^{a}$ ) is a directed supremum of such formal balls $(\xi, r)$ way-below it, which therefore form a basis.

Remark 8.13. Let $X$ be a poset. A root for $d_{\leq}$is the same thing as a least element $\perp$. Hence, using the same arguments as in Remark 8.4 on the $d_{K R}$ quasi-metric, Theorem 8.9 implies that for every pointed continuous dcpo, $\mathbf{V}_{1} X$ is a continuous dcpo. This result is due to Edalat [9, Section 3].

Considering $d_{K R}^{1}$ instead, we obtain that, if $X$ is a pointed continuous dcpo, then $\mathbf{V}_{1} X$ is a rooted, continuous complete quasi-metric space in Sünderhauf's sup quasi-metric. 


\section{The weak topology}

Recall that the weak topology on $\mathbf{V}_{*} X$ has subbasic open sets $[h>b]=$ $\left\{\nu \in \mathbf{V}_{*} X \mid \int_{x \in X} h(x) d \nu>b\right\}, h \in \mathcal{L} X, b \in \mathbb{R}_{+}$.

When $X, d$ is a standard quasi-metric space, another subbase for the weak topology is given by the open sets $[h>b]$, where now $h$ is restricted to be 1-Lipschitz continuous. This is because, for every $h \in \mathcal{L} X, h=\sup _{\alpha>0} h^{(\alpha)}$, so $[h>b]=\bigcup_{\alpha>0}\left[1 / \alpha h^{(\alpha)}>b / \alpha\right]$.

By the same argument, this time using the fact that $h=\sup _{\beta>0} \min (h, \beta)$, hence that $[h>b]=\bigcup_{\beta>0}[\min (h, \beta)>b]$, the open sets $[h>b]$ also form a subbase of the weak topology, where $h$ is now bounded and in $\mathcal{L} X$.

Now fix $a \in \mathbb{R}_{+}, a>0$. For $h$ bounded in $\mathcal{L} X, h$ is the directed supremum of bounded Lipschitz continuous maps $h^{(\alpha)}, \alpha>0$, so the same argument shows that an even smaller subbase is given by the sets $[h>b]$ where $h \in$ $\mathcal{L}_{\infty}^{\mathrm{b}}(X, d)$. By Lemma 3.16, $h$ is in $\mathcal{L}_{\alpha}^{a}(X, d)$ for some $\alpha>0$. Then $[h>$ $b]=[1 / \alpha h>b / \alpha]$, so that we can take the sets $[h>b]$ as a subbase of the weak topology, where $h$ is now in $\mathcal{L}_{1}^{a}(X, d)$. This will be used in the next proposition.

Proposition 9.1. Let $*$ be nothing, " $\leq 1$ ", or " 1 ". Let $X, d$ be a standard quasi-metric space, $a, a^{\prime}>0$ with $a \leq a^{\prime}$, and assume that the spaces $\mathbf{V}_{*} X, d_{K R}^{a}, \mathbf{V}_{*} X, d_{K R}^{a^{\prime}}$ and $\mathbf{V}_{*} X, d_{K R}$ are complete and that directed suprema in their spaces of formal balls are computed as naive suprema.

Then we have the following inclusions of topologies on $Y$ :

$$
\text { weak } \subseteq d_{K R^{-}}^{a} S \operatorname{cott} \subseteq d_{K R^{-}}^{a^{\prime}} S \operatorname{cott} \subseteq d_{K R^{-}} \text {Scott }
$$

Proof. First inclusion. We use yet another topology, this time on $\mathbf{B}\left(\mathbf{V}_{*} X\right.$, $\left.d_{\mathrm{KR}}^{a}\right)$. Let $[h>b]^{+}$be defined as the set of those formal balls $(\nu, r)$ with $\nu \in \mathbf{V}_{*} X$ such that $\int_{x \in X} h(x) d \nu>r+b$. The weak $k^{+}$topology on $\mathbf{B}\left(\mathbf{V}_{*} X, d_{\mathrm{KR}}^{a}\right)$ has a subbase of open sets given by the sets $[h>b]^{+}$, for every $h \in \mathcal{L}_{1}^{a}(X, d)$ and $b \in \mathbb{R}_{+}$.

$[h>b]^{+}$is upwards-closed in $\mathbf{B}\left(\mathbf{V}_{*} X, d_{\mathrm{KR}}^{a}\right)$. Indeed, assume that $(\nu, r) \in$ $[h>b]^{+}$, where $h \in \mathcal{L}_{1}^{a}(X, d)$, and that $(\nu, r) \leq d_{\mathrm{KR}}^{a+}\left(\nu^{\prime}, r^{\prime}\right)$. By Lemma 3.10,

item $2, \int_{x \in X} h(x) d \nu-r \leq \int_{x \in X} h(x) d \nu^{\prime}-r^{\prime}$. Since $\int_{x \in X} h(x) d \nu>r+b$, $\int_{x \in X} h(x) d \nu^{\prime}>r^{\prime}+b$, namely $\nu^{\prime} \in[h>b]^{+}$.

To show that $[h>b]^{+}$is Scott-open in $\mathbf{B}\left(\mathbf{V}_{*} X, d_{\mathrm{KR}}^{a}\right)$, let $\left(\nu_{i}, r_{i}\right)_{i \in I}$ be a directed family with (naive) supremum $(\nu, r)$ in $\mathbf{B}\left(\mathbf{V}_{*} X, d_{\mathrm{KR}}^{a}\right)$ and assume 
that $(\nu, r) \in[h>b]^{+}$. Since $\int_{x \in X} h(x) d \nu=\sup _{i \in I}\left(\int_{x \in X} h(x) d \nu_{i}+r-r_{i}\right)>$ $r+b, \int_{x \in X} h(x) d \nu_{i}>r_{i}+b$ for some $i \in I$, i.e., $\left(\nu_{i}, r_{i}\right)$ is in $[h>b]^{+}$.

We can now proceed to show that $[h>b]$ is $d_{\mathrm{KR}}^{a}$-Scott open, for every $h \in \mathcal{L}_{1}^{a}(X, d)$. Equating $\mathbf{V}_{*} X$ with the subset of all formal balls $(\nu, 0)$, $\nu \in \mathbf{V}_{*} X,[h>b]$ is equal to $\mathbf{V}_{*} X \cap[h>b]^{+}$. Since $[h>b]^{+}$is Scott-open, $[h>b]$ is $d_{\mathrm{KR}}^{a}$-Scott open.

Second and third inclusions. The proofs of the second and third inclusions are similar. We rely on the easily checked inequalities $d_{\mathrm{KR}}^{a} \leq d_{\mathrm{KR}}^{a^{\prime}} \leq d_{\mathrm{KR}}$, for $a \leq a^{\prime}$. This implies that: $(*)(\nu, r) \leq d_{\mathrm{KR}}^{+}\left(\nu^{\prime}, s\right)$ implies $(\nu, r) \leq \underbrace{a^{\prime}+}_{\mathrm{KR}}\left(\nu^{\prime}, s\right)$, and that $(\nu, r) \leq d_{\mathrm{KR}}^{a^{\prime}+}\left(\nu^{\prime}, s\right)$ implies $(\nu, r) \leq d_{\mathrm{KR}}^{a+}\left(\nu^{\prime}, s\right)$.

Let $\mathcal{U}$ be a Scott-open subset of $\mathbf{B}\left(\mathbf{V}_{*} X, d_{\mathrm{KR}}^{a}\right)$. Since $\mathcal{U}$ is upwards-closed in $\leq d_{\mathrm{KR}}^{a+}$, it is also upwards-closed in $\leq d_{\mathrm{KR}}^{a^{\prime}+}$ by $(*)$. Assume now that $(\nu, r) \in \mathcal{U}$ is the supremum in $\mathbf{B}\left(\mathbf{V}_{*} X, d_{\mathrm{KR}}^{a^{\prime}}\right)$ of a family $\left(\nu_{i}, r_{i}\right)_{i \in I}$ that is directed with respect to $\leq d_{\mathrm{KR}}^{a^{\prime}+}$. By $(*)$ again, $\left(\nu_{i}, r_{i}\right)_{i \in I}$ is directed with respect to $\leq d_{\mathrm{KR}}^{a+}$. Therefore $\left(\nu_{i}, r_{i}\right)_{i \in I}$ has a supremum $\left(\nu^{\prime}, r^{\prime}\right)$ in $\mathbf{B}\left(\mathbf{V}_{*} X, d_{\mathrm{KR}}^{a}\right)$ that is uniquely characterized by $r^{\prime}=\inf _{i \in I} r_{i}, \int_{x \in X} h(x) d \nu^{\prime}=\sup _{i \in I}\left(\int_{x \in X} h(x) d \nu_{i}+r-r_{i}\right)$ for every $h \in \mathcal{L}_{1}^{a}(X, d)$, since suprema are assumed to be naive. However, by naivety again, $r=\inf _{i \in I} r_{i}$ and $\int_{x \in X} h(x) d \nu=\sup _{i \in I}\left(\int_{x \in X} h(x) d \nu_{i}+r-r_{i}\right)$ for every $h \in \mathcal{L}_{1}^{a^{\prime}}(X, d)$. This holds in particular for every $h \in \mathcal{L}_{1}^{a}(X, d)$, so $\int_{x \in X} h(x) d \nu=\int_{x \in X} h(x) d \nu^{\prime}$ for every $h \in \mathcal{L}_{1}^{a}(X, d)$, whence $\nu=\nu^{\prime}$ by Corollary 3.19. We have therefore obtained that $(\nu, r)$ is also the supremum of the directed family $\left(\nu_{i}, r_{i}\right)_{i \in I}$ in $\mathbf{B}\left(\mathbf{V}_{*} X, d_{\mathrm{KR}}^{a}\right)$. Since $(\nu, r) \in \mathcal{U}$, some $\left(\nu_{i}, r_{i}\right)$ is also in $\mathcal{U}$ since $\mathcal{U}$ is Scott-open in $\mathbf{B}\left(\mathbf{V}_{*} X, d_{\mathrm{KR}}^{a}\right)$. This shows that $\mathcal{U}$ is Scott-open in $\mathbf{B}\left(\mathbf{V}_{*} X, d_{\mathrm{KR}}^{a^{\prime}}\right)$. Similarly, we show that every Scott-open subset of $\mathbf{B}\left(\mathbf{V}_{*} X, d_{\mathrm{KR}}^{a^{\prime}}\right)$ is Scott-open in $\mathbf{B}\left(\mathbf{V}_{*} X, d_{\mathrm{KR}}\right)$, using Corollary 3.14 instead of Corollary 3.19.

By taking intersections $\mathcal{U} \cap \mathbf{V}_{*} X$, it follows that every $d_{\mathrm{KR}}^{a}-$ Scott open is $d_{\mathrm{KR}}^{a^{\prime}}$-Scott open, and that every $d_{\mathrm{KR}}^{a^{\prime}}$-Scott open is $d_{\mathrm{KR}}$-Scott open.

The assumptions of Proposition 9.1 are in particular satisfied when $X, d$ is continuous complete, by Theorem 4.15.

We will see that the $d_{\mathrm{KR}}$-Scott topology is in general strictly finer than the other topologies (Remark 9.4). We will also see that the other topologies are all equal, assuming $X, d$ algebraic.

Lemma 9.2. Let $Y, \partial$ be a standard algebraic quasi-metric space, and $\mathcal{B}$ be a strong basis of $Y, \partial$. Fix also $\epsilon>0$. Every $\partial$-Scott open subset of $Y$ is a union of open balls $B_{b,<r}^{\partial}$, where $b \in \mathcal{B}$ and $0<r<\epsilon$. 
Proof. Let $V$ be a $\partial$-Scott open subset of $Y$, and $y \in V$. Our task is to show that $y \in B_{b,<r}^{\partial} \subseteq V$ for some $b \in \mathcal{B}$ and $r>0$ such that $r<\epsilon$. By definition of the $\partial$-Scott topology, $V$ is the intersection of the Scott-open subset $\widehat{V}$ of $\mathbf{B}(Y, \partial)$ with $Y$. Since $X, d$ is standard, $V_{\epsilon}$ is also Scott-open, and $V$ is also the intersection of $\widehat{V} \cap V_{\epsilon}$ with $Y$. ( $\widehat{V}$ is the largest Scott-open subset of $\mathbf{B}(Y, \partial)$ whose intersection with $Y$ equals $V$.) We use Lemma 5.6 and the fact that $(y, 0)$ is in $\widehat{V} \cap V_{\epsilon}$ to conclude that there is a $b \in \mathcal{B}$ and an $r \in \mathbb{R}_{+}$such that $(b, r) \ll(y, 0)$ and $(b, r)$ is in $\widehat{V} \cap V_{\epsilon}$. Since $(b, r) \ll(y, 0)$, $\partial(b, y)<r$, which implies in particular that $r>0$, and also that $y$ is in $B_{b,<r}^{\partial}$. Since $(b, r)$ is in $V_{\epsilon}, r<\epsilon$. Finally, $B_{b,<r}^{\partial}$ is included in $V$ : for every $z \in B_{b,<r}^{\partial}, d(b, z)<r$, so $(b, r) \ll(z, 0)$, using the final part of Lemma 5.6. That shows that $(z, 0)$ is in $\uparrow(b, r) \subseteq \widehat{V}$.

Proposition 9.3. Let $X, d$ be an algebraic complete quasi-metric space. For every $a \in \mathbb{R}_{+}, a>0$, the $d_{K R}^{a}$-Scott topology coincides with the weak topology on $\mathbf{V}_{\leq 1} X$, and on $\mathbf{V}_{1} X$.

Proof. The $d_{\mathrm{KR}}^{a}$-Scott topology is finer by Proposition 9.1. Conversely, let $\nu \in \mathbf{V}_{\leq 1} X$ (resp., $\mathbf{V}_{1} X$ ) and $\mathcal{U}$ be a $d_{\mathrm{KR}}^{a}$ Scott open neighborhood of $\nu$. We wish to show that $\nu$ is in some weak open subset of $\mathcal{U}$. By Theorem 5.7 (resp., Theorem 6.1), $\mathbf{V}_{\leq 1} X, d_{\mathrm{KR}}^{a}$ (resp., $\mathbf{V}_{1} X, d_{\mathrm{KR}}^{a}$ ) is algebraic, and we can then apply Lemma 9.2 , taking $\mathcal{B}$ equal to the subset of simple (subprobability, resp. probability) valuations $\sum_{i=1}^{n} a_{i} \delta_{x_{i}}$ where each $x_{i}$ is a center point. Therefore we can assume that $\mathcal{U}=B_{\sum_{i=1}^{n} a_{i} \delta_{x_{i}},<\epsilon}^{d_{\mathrm{R}}^{a}}$ for some such simple valuation $\sum_{i=1}^{n} a_{i} \delta_{x_{i}}$ and some $\epsilon>0$.

By assumption, $d_{\mathrm{KR}}^{a}\left(\sum_{i=1}^{n} a_{i} \delta_{x_{i}}, \nu\right)<\epsilon$. Let $\eta>0$ be chosen such that $d_{\mathrm{KR}}^{a}\left(\sum_{i=1}^{n} a_{i} \delta_{x_{i}}, \nu\right)<\epsilon-\eta$. Let $N$ be a natural number such that $a / N<\eta$, and let us consider the collection $\mathcal{H}$ of maps of the form $\bigvee_{i=1}^{n} x_{i} \nearrow b_{i}$ (see Proposition 6.12) where each $b_{i}$ is an integer multiple of $a / N$ in $[0, a]$. Note that $\mathcal{H}$ is a finite family, and that for each $h \in \mathcal{H}, \sum_{i=1}^{n} a_{i} h\left(x_{i}\right)<+\infty$.

Let $\mathcal{V}$ be the weak open set $\bigcap_{h \in \mathcal{H}}\left[h>\sum_{i=1}^{n} a_{i} h\left(x_{i}\right)-\epsilon+\eta\right]$. (We extend the notation $[h>b]$ to the case where $b<0$ in the obvious way, as the set of continuous valuations $\nu^{\prime}$ such that $\int_{x \in X} h(x) d \nu^{\prime}>b$; when $b<0$, this is the whole set, hence is again open.) For every $h \in \mathcal{H}, h$ is in $\mathcal{L}_{1}^{a}(X, d)$ : it is in $\mathcal{L}_{1}(X, d)$ by Lemma 6.10 , and clearly bounded from above by $a$. Since $d_{\mathrm{KR}}^{a}\left(\sum_{i=1}^{n} a_{i} \delta_{x_{i}}, \nu\right)<\epsilon-\eta, \sum_{i=1}^{n} a_{i} h\left(x_{i}\right)<\int_{x \in X} h(x) d \nu+\epsilon-\eta$. Hence $\nu$ is in $\mathcal{V}$. 
Next, we show that $\mathcal{V}$ is included in $\mathcal{U}=B_{\sum_{i=1}^{n} a_{i} \delta_{x_{i}},<\epsilon}^{a}$. Let $\nu^{\prime}$ be an arbitrary element of $\mathcal{V}$. By Proposition 6.12 (second part), there are numbers $b_{i}^{\prime} \in$ $[0, a], 1 \leq i \leq n$, such that $d_{\mathrm{KR}}^{a}\left(\sum_{i=1}^{n} a_{i} \delta_{x_{i}}, \nu^{\prime}\right)=\mathrm{d}_{\mathbb{R}}\left(\sum_{i=1}^{n} a_{i} h^{\prime}\left(x_{i}\right), \int_{x \in X} h^{\prime}(x) d \nu^{\prime}\right)$, where $h^{\prime}=\bigvee_{i=1}^{n} x_{i} \nearrow b_{i}^{\prime}$. For each $i$, let $b_{i}$ be the largest integer multiple of $a / N$ below $b_{i}^{\prime}$, and let $h=\bigvee_{i=1}^{n} x_{i} \nearrow b_{i}$. Since $h$ is in $\mathcal{H}$, and $\nu^{\prime} \in \mathcal{V}$, $\sum_{i=1}^{n} a_{i} h\left(x_{i}\right)<\int_{x \in X} h(x) d \nu^{\prime}+\epsilon-\eta$. For each $i, b_{i}^{\prime} \leq b_{i}+a / N \leq b_{i}+\eta$. It follows that, for every $x \in X,\left(x_{i} \nearrow b_{i}^{\prime}\right)(x)=\max \left(b_{i}^{\prime}-d\left(x_{i}, x\right), 0\right) \leq \max \left(b_{i}+\eta-\right.$ $\left.d\left(x_{i}, x\right), 0\right) \leq \max \left(b_{i}-d\left(x_{i}, x\right), 0\right)+\eta=\left(x_{i} \nearrow b_{i}\right)(x)+\eta$. In turn, we obtain that for every $x \in X, h^{\prime}(x) \leq h(x)+\eta$, so $\sum_{i=1}^{n} a_{i} h^{\prime}\left(x_{i}\right) \leq \sum_{i=1}^{n} a_{i} h\left(x_{i}\right)+\eta$, using the fact that $\sum_{i=1}^{n} a_{i} \leq 1$. Therefore $\sum_{i=1}^{n} a_{i} h^{\prime}\left(x_{i}\right)<\int_{x \in X} h(x) d \nu^{\prime}+\epsilon$. This implies that $d_{\mathrm{KR}}^{a}\left(\sum_{i=1}^{n} a_{i} \delta_{x_{i}}, \nu^{\prime}\right)<\epsilon$. Therefore $\nu^{\prime}$ is in $\mathcal{U}$.

Remark 9.4. As a special case of Proposition 9.3, applied to complete metric spaces $X, d$, the $d_{K R}^{a}$-Scott topology on $\mathbf{V}_{1} X$ (resp., $\mathbf{V}_{\leq 1} X$ ) coincides with the weak topology. Since the former is the usual open ball topology of the metric $d_{K R}^{a}, d_{K R}^{a}$ metrizes the weak topology on spaces of (sub)probability valuations on complete metric spaces. This subsumes the well-known result that it metrizes the weak topology on spaces of probability measures on complete separable (Polish) spaces. Recall that on Polish spaces, and more generally, on quasi-Polish spaces, bounded measures and bounded continuous valuations are in bijective correspondence.

However, the unbounded $d_{K R}$ metric does not metrize the weak topology. For a counterexample, we reuse one due to Kravchenko [26, Lemma 3.7]. Take any complete metric space $X, d$, with points $\left(x_{n}\right)_{n \in \mathbb{N} \backslash\{0\}}$ such that $n \leq$ $d\left(x_{0}, x_{n}\right)<+\infty$. Let $\nu_{n}=\frac{1}{n} \delta_{x_{n}}+\left(1-\frac{1}{n}\right) \delta_{x_{0}}$. Then $\left(\nu_{n}\right)_{n \in \mathbb{N} \backslash\{0\}}$ converges to $\delta_{x_{0}}$ in the weak topology, since for every subbasic weak open set $[f>r]$ containing $\delta_{x_{0}}$ (i.e., $f\left(x_{0}\right)>r$ ), $\int_{x \in X} f(x) d \nu_{n}=\frac{1}{n} f\left(x_{n}\right)+\left(1-\frac{1}{n}\right) f\left(x_{0}\right)$ is strictly larger than $r$ for $n$ large enough (this is easy if $r \geq 0$ since then $f\left(x_{0}\right)>0$, and is trivial if $r<0$ since $f \in \mathcal{L} X$ takes its values in $\left.\overline{\mathbb{R}}_{+}\right)$. However, $d_{K R}\left(\delta_{x_{0}}, \nu_{n}\right)=\sup _{h \in \mathcal{L}_{1} X} \mathrm{~d}_{\mathbb{R}}\left(h\left(x_{0}\right), \frac{1}{n} h\left(x_{n}\right)+\left(1-\frac{1}{n}\right) h\left(x_{0}\right)\right) \geq$ $\mathrm{d}_{\mathbb{R}}\left(d\left(x_{0}, x_{n}\right),\left(1-\frac{1}{n}\right) d\left(x_{0}, x_{n}\right)\right)$ (taking $\left.h=d\left({ }_{-}, x_{n}\right)\right)=\frac{1}{n} d\left(x_{0}, x_{n}\right) \geq 1$, which shows that $\left(\nu_{n}\right)_{n \in \mathbb{N} \backslash\{0\}}$ does not converge to $\delta_{x_{0}}$ in the open ball topology of $d_{K R}$.

We reassure ourselves in checking that $\left(\nu_{n}\right)_{n \in \mathbb{N} \backslash\{0\}}$ does converge to $\delta_{x_{0}}$ in the open ball topology of $d_{K R}^{a}$, though (this must be, since we announced that it would coincide with the weak topology $): d_{K R}^{a}\left(\delta_{x_{0}}, \nu_{n}\right)=\sup _{h \in \mathcal{L}_{1}^{a} X} \max \left(\frac{1}{n}\left(h\left(x_{0}\right)-\right.\right.$ $\left.\left.h\left(x_{n}\right)\right)\right) \leq \frac{a}{n}$. 
Theorem 9.5 ( $d_{\mathbf{K R}}^{a}$ quasi-metrizes the weak topology). Let $X, d$ be a continuous complete quasi-metric space. For every $a \in \mathbb{R}_{+}, a>0$, the $d_{K R^{-}}^{a}$ Scott topology coincides with the weak topology on $\mathbf{V}_{\leq 1} X$, and on $\mathbf{V}_{1} X$.

PROOF. We reduce to the case of algebraic complete quasi-metric spaces.

We invoke [20, Theorem 7.9] again: $X, d$ is the 1-Lipschitz continuous retract of an algebraic complete quasi-metric space $Y, \partial$. Call $s: X \rightarrow Y$ the section and $r: Y \rightarrow X$ the retraction. Then $\mathbf{V} s$ and $\mathbf{V} r$ form a 1Lipschitz continuous section-retraction pair by Lemma 4.8, and in particular $\mathbf{V} s$ is an embedding of $\mathbf{V}_{\leq 1} X$ into $\mathbf{V}_{\leq 1} Y$ with their $d_{\mathrm{KR}}^{a}$-Scott topologies (similarly with $\mathbf{V}_{1}$ ). However, $s$ and $r$ are also just continuous, since every 1Lipschitz continuous map between standard spaces is, so $\mathbf{V} s$ and $\mathbf{V} r$ also form a section-retraction pair between the same spaces, this time with their weak topologies. (Indeed, note that whenever $f$ is continuous, so is $\mathbf{V} f$, because $\left.(\mathbf{V} f)^{-1}([h>b])=[h \circ f>b].\right)$ By Proposition 9.3, the two topologies on $\mathbf{V}_{\leq 1} Y$ (resp., $\mathbf{V}_{1} Y$ ) are the same. However, $\mathbf{V}_{\leq 1} X$ (resp., $\mathbf{V}_{1} X$ ) embeds in the latter, both in its $d_{\mathrm{KR}}^{a}$-Scott topology and in its weak topology, through the topological embedding $\mathbf{V} s$. Hence the two topologies on $\mathbf{V}_{\leq 1} X$ (resp., $\left.\mathbf{V}_{1} X\right)$ are the same as well.

\section{Open Questions}

1. Assume $X, d$ standard algebraic. Is $\mathbf{V}_{1} X, d_{\mathrm{KR}}$ algebraic? This is the case if $X$ has a root (Theorem 6.6), in particular if $d$ is bounded. Close results are that $\mathbf{V}_{1} X, d_{\mathrm{KR}}^{a}$ is algebraic for every $a>0$ (Theorem 6.1), and that $\mathbf{V}_{\leq 1} X, d_{\mathrm{KR}}$ is algebraic (Theorem 5.7). Beware of Kravchenko's counterexample: the $d_{\mathrm{KR}}$-Scott topology on $\mathbf{V}_{1} X$ is in general different from the weak topology, and the coincidence with the weak topology in the rooted and $d_{\mathrm{KR}}^{a}$ cases followed more or less directly from algebraicity.

2. The above theorems apply to spaces of normalized, or subnormalized valuations. Are there analogous results for the space $\mathbf{V} X$ of all continuous valuations? I doubt it, since, by analogy, the space of all measures on a Polish space is in general not even metrizable.

3. Does the Kantorovich-Rubinstein duality theorem extend to interesting classes of non-metric quasi-metric spaces? As we have already said in the related work section, such a result was announced for symcompact 
spaces [15, Theorem 5]. One would hope a form of it for quasi-Polish spaces, or more precisely, for second countable Smyth-complete quasimetric spaces. Alternatively, can one imagine doing an independent study of $W^{p}$-like quasi-metrics on such spaces?

The difficulty, which may appear silly at first but runs deep, is that the map $(x, y) \mapsto d(x, y)$ is not lower semicontinuous, because it is antitonic in $y$. It is not even Borel measurable in general, so that

the integral $\int_{(x, y) \in X^{2}} d(x, y) d \varpi$ defining $W^{1}$ does not make sense. For a counterexample, consider the $d_{\leq}$metric on $\omega_{1}+1$, where $\omega_{1}$ is the

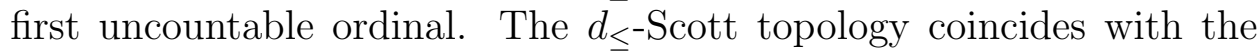
Scott topology (see Remark 3.3). The family of countable subsets of $\omega_{1}+1$ that do not contain $\omega_{1}$ and their complements forms a $\sigma$-algebra containing all the Scott-open sets, so $\left\{\omega_{1}\right\}$ is not Borel. But $\left\{\omega_{1}\right\}$ is the inverse image of the closed set $[0,1]$ by the map $y \mapsto d_{\leq}\left(\omega_{1}, y\right)$. Hence $y \mapsto d_{\leq}\left(\omega_{1}, y\right)$ is not Borel measurable, and therefore neither is $d_{\leq}$.

\section{References}

[1] Adamski, W., 1977. $\tau$-smooth Borel measures on topological spaces. Mathematische Nachrichten 78, 97-107.

[2] Bolley, F., 2008. Separability and completeness for the Wasserstein distance. In: Séminaire de probabilités XLI. Vol. 1934 of Lecture Notes in Mathematics. Springer Verlag, pp. 371-377.

[3] Bonsangue, M. M., van Breugel, F., Rutten, J. J. M. M., 1998. Generalized metric spaces: Completion, topology, and powerdomains via the Yoneda embedding. Theoretical Computer Science 193, 1-51.

[4] Choquet, G., 1953-54. Theory of capacities. Annales de l'Institut Fourier $5,131-295$.

[5] de Acosta, A. D., 1982. Invariance principles in probability for triangular arrays of B-valued random vectors and some applications. Annals of Probability 10 (2).

[6] de Brecht, M., 2013. Quasi-Polish spaces. Annals of Pure and Applied Logic 164 (3), 356-381. 
[7] de Brecht, M., Goubault-Larrecq, J., Jia, X., Lyu, Z., 2019. Domaincomplete and LCS-complete spaces. Electronic Notes in Theoretical Computer Science 345, 3-35, proc. 8th International Symposium on Domain Theory (ISDT'19), also available as arXiv report 1902.11142 [math.GN].

[8] Dudley, R. M., 1976. Probabilities and Metrics. Vol. 45 of Lecture Notes. Matematisk Institut, Aarhus University, Aarhus.

[9] Edalat, A., 1995. Domain theory and integration. Theoretical Computer Science 151, 163-193.

[10] Edalat, A., Heckmann, R., 1998. A computational model for metric spaces. Theoretical Computer Science 193, 53-73.

[11] Escardó, M. H., 1998. Properly injective spaces and function spaces. Topology and Its Applications 89 (1-2).

[12] Fernique, X., 1981. Sur le théorème de Kantorovitch-Rubinstein dans les espaces polonais. Séminaire de probabilités (Strasbourg) 15, 6-10.

[13] Gierz, G., Hofmann, K. H., Keimel, K., Lawson, J. D., Mislove, M., Scott, D. S., 2003. Continuous Lattices and Domains. Vol. 93 of Encyclopedia of Mathematics and its Applications. Cambridge University Press.

[14] Goubault-Larrecq, J., Sep. 2007. Continuous previsions. In: Duparc, J., Henzinger, T. A. (Eds.), Proceedings of the 16th Annual EACSL Conference on Computer Science Logic (CSL'07). Springer-Verlag LNCS 4646, Lausanne, Switzerland, pp. 542-557.

[15] Goubault-Larrecq, J., Mar.-Apr. 2008. Simulation hemi-metrics between infinite-state stochastic games. In: Amadio, R. (Ed.), Proceedings of the 11th International Conference on Foundations of Software Science and Computation Structures (FoSSaCS'08). Springer-Verlag LNCS 4962, Budapest, Hungary, pp. 50-65.

[16] Goubault-Larrecq, J., 2013. Non-Hausdorff Topology and Domain Theory-Selected Topics in Point-Set Topology. Vol. 22 of New Mathematical Monographs. Cambridge University Press. 
[17] Goubault-Larrecq, J., 2017. Complete quasi-metrics for hyperspaces, continuous valuations, and previsions, arXiv 1707.03784, v2, https://arxiv.org/abs/1707.03784; v6, July 2020.

[18] Goubault-Larrecq, J., 2019. Formal ball monads. Topology and its Applications 263, 372-391.

[19] Goubault-Larrecq, J., 2020. Some topological properties of spaces of Lipschitz continuous maps on quasi-metric spaces. Topology and its Applications. 282, available online 1 June 2020, 107281, https://doi.org/10.1016/j.topol.2020.107281.

[20] Goubault-Larrecq, J., Ng, K. M., Oct. 2017. A few notes on formal balls. Logical Methods in Computer Science 13 (4:18), 1-34, special issue of Domain Theory and Applications (the Domains XII Workshop), Cork, Ireland, August 2015.

[21] Jones, C., 1990. Probabilistic non-determinism. Ph.D. thesis, University of Edinburgh, technical Report ECS-LFCS-90-105.

[22] Jones, C., Plotkin, G. D., 1989. A probabilistic powerdomain of evaluations. In: Proc. 4th IEEE Symposium on Logics in Computer Science (LICS'89). IEEE Computer Society Press, pp. 186-195.

[23] Kantorovich, L. V., 1942. On the translocation of masses. Comptes Rendus (Doklady) de l'Acad. Sci. URSS 37, 199-201, reprinted in Management Science, vol. 5, 1-4, 1958.

[24] Kantorovich, L. V., Rubinshteĭn, G. S., 1958. On a space of totally additive functions. Vestnik Leningradskogo Universiteta 13 (7), 52-59.

[25] Kostanek, M., Waszkiewicz, P., 2010. The formal ball model for $\mathcal{Q}$ categories. Mathematical Structures in Computer Science 21 (1), 1-24.

[26] Kravchenko, A. S., 2006. Completeness of the space of separable measures in the Kantorovich-Rubinshteĭn metric. Siberian Mathematical Journal 47 (1), 68-76.

[27] Ng, K. M., Ho, W. K., 2019. Quasi-continuous Yoneda complete quasi-metric spaces. Electronic Notes in Theoretical Computer Science 345, 185-217, proc. 8th International Symposium on Domain Theory (ISDT'19). 
[28] Prokhorov, Y. V., 1956. Convergence of random processes and limit theorems in probability theory. Theory of Probability and its Applications $1,157-214$.

[29] Sünderhauf, P., 1998. Spaces of valuations as quasimetric domains. Electronic Notes in Theoretical Computer Science 13, 183-196.

[30] Tix, R., 1995. Stetige Bewertungen auf topologischen Räumen. Diplomarbeit, TH Darmstadt.

[31] Weihrauch, K., Schreiber, U., 1981. Embedding metric spaces into cpo's. Theoretical Computer Science 16 (1), 5-24. 